Agrupamento de dados fuzzy colaborativo

\author{
Luiz Fernando Sommaggio Coletta
}



SERVIÇO DE PÓS-GRADUAÇÃO DO ICMC-USP

Data de Depósito:

Assinatura:

\title{
Agrupamento de dados fuzzy colaborativo
}

\author{
Luiz Fernando Sommaggio Coletta
}

Orientador: Prof. Dr. Eduardo Raul Hruschka

Dissertação apresentada ao Instituto de Ciências Matemáticas e de Computação - ICMC-USP, como parte dos requisitos para obtenção do título de Mestre em Ciências - Ciências de Computação e Matemática Computacional. VERSÃO REVISADA

USP - São Carlos

Abril de 2011 
Ficha catalográfica elaborada pela Biblioteca Prof. Achille Bassi e Seção Técnica de Informática, ICMC/USP, com os dados fornecidos pelo(a) autor(a)

Sommaggio Coletta, Luiz Fernando
Agrupamento de Dados Fuzzy Colaborativo / Luiz
Fernando Sommagio Coletta; orientador Eduardo Raul
Hruschka -- São Carlos, 2011.
95 p.
Dissertação (Mestrado - Programa de Pós-Graduação en
Ciências de Computação e Matemática Computacional) --
Instituto de Ciências Matemáticas e de Computação,
Universidade de São Paulo, 2011.
1. Mineração de Dados. 2. Agrupamento de Dados
Colaborativo. I. Raul Hruschka, Eduardo, orient. II.
Título.


Feliz aquele que transfere o que sabe e aprende o que ensina

(Cora Coralina) 


\section{Agradecimentos}

Esta dissertação é fruto de um trabalho conjunto de pesquisa. Nesse sentido, inicialmente gostaria de agradecer aos Professores Ricardo J. G. B. Campello (ICMC/USP) e Witold Pedrycz (University of Alberta, Canada), bem como ao amigo Lucas Vendramin (ICMC/USP), pelas inúmeras discussões técnicas que tivemos e pela participação ativa no desenvolvimento de várias das idéias abordadas nessa dissertação. Em particular, gostaria de registrar meu reconhecimento ao Prof. Ricardo J. G. B. Campello, que na prática atuou como meu co-orientador, pelo seu esforço em acompanhar cuidadosamente todas as fases de desenvolvimento desse trabalho. Gostaria de agradecer com ênfase ao meu orientador, Prof. Eduardo Raul Hruschka, por estar sempre presente com inteligência, dedicação, paciência e muito bom humor, qualidades que o tornam não apenas um excelente professor e orientador, mas também um grande amigão. Sou grato também aos meus pais, Maria e Edson, e a toda a minha família pelo incentivo, apoio e conforto durante os anos de estudo. Gostaria de deixar meus agradecimentos também aos companheiros de trabalho, que de alguma forma colaboraram com esta pesquisa. Em especial, aos colegas Bruno Feres, Danilo Horta, Débora Medeiros, Jonathan Andrade, Murilo Naldi, Pablo Andretta, Rafael Rossi, Ricardo Cerri, Ricardo Marcacini e Thiago Covões. Por fim, agradeço a todos os funcionários do ICMC/USP pela dedicação e competência e à FAPESP pelo suporte financeiro. 


\section{Resumo}

Nas últimas décadas, as técnicas de mineração de dados têm desempenhado um importante papel em diversas áreas do conhecimento humano. Mais recentemente, essas ferramentas têm encontrado espaço em um novo e complexo domínio, no qual os dados a serem minerados estão fisicamente distribuídos. Nesse domínio, alguns algoritmos específicos para agrupamento de dados podem ser utilizados - em particular, algumas variantes do algoritmo amplamente conhecido Fuzzy C-Means (FCM), as quais têm sido investigadas sob o nome de agrupamento fuzzy colaborativo. Com o objetivo de superar algumas das limitações encontradas em dois desses algoritmos, cinco novos algoritmos foram desenvolvidos nesse trabalho. Esses algoritmos foram estudados em dois cenários específicos de aplicação que levam em conta duas suposições sobre os dados (i.e., se os dados são de uma mesma população ou de diferentes populações). $\mathrm{Na}$ prática, tais suposições e a dificuldade em se definir alguns dos parâmetros (que possam ser requeridos), podem orientar a escolha feita pelo usuário entre os algoritmos disponíveis. Nesse sentido, exemplos ilustrativos destacam as diferenças de desempenho entre os algoritmos estudados e desenvolvidos, permitindo derivar algumas conclusões que podem ser úteis ao aplicar agrupamento fuzzy colaborativo na prática. Análises de complexidade de tempo, espaço e comunicação também foram realizadas. 


\section{Abstract}

Data mining techniques have played an important role in several areas of human knowledge. More recently, these techniques have found space in a new and complex setting in which the data to be mined are physically distributed. In this setting, some specific algorithms for data clustering can be used, such as some variants of the widely used Fuzzy C-Means (FCM) algorithm that support clustering data distributed across different sites. Those methods have been studied under different names, like collaborative and parallel fuzzy clustering. In this study, we offer some augmentation of the two FCM-based clustering algorithms used to cluster distributed data by arriving at some constructive ways of determining essential parameters of the algorithms (including the number of clusters) and forming a set of systematically structured guidelines as to a selection of the specific algorithm depending upon a nature of the data environment and the assumptions being made about the number of clusters. A thorough complexity analysis including space, time, and communication aspects is reported. A series of detailed numeric experiments is used to illustrate the main ideas discussed in the study. 


\section{Sumário}

Lista de Figuras . . . . . . . . . . . . . . . . . . . . xv

Lista de Tabelas . . . . . . . . . . . . . . . . . . xvii

Lista de Abreviaturas ..................... . . . . . . . . . .

1 Introdução 1

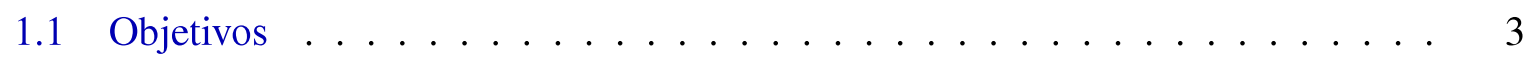

1.2 Organização da Dissertação . . . . . . . . . . . . . . . . . 3

2 Agrupamento de Dados 5

2.1 Complexidade do Problema . . . . . . . . . . . . . . . 7

2.2 Algoritmo K-Means . . . . . . . . . . . . . . . . 7

2.3 Algoritmo Fuzzy C-Means . . . . . . . . . . . . . . . . . . 8

2.4 Validação de Agrupamentos de Dados . . . . . . . . . . . . . . . . . . 9

2.4.1 Silhueta Crisp . . . . . . . . . . . . . . . . 10

2.4 .2 Silhueta Fuzzy . . . . . . . . . . . . . . . . . . . 11

2.4.3 Silhueta Fuzzy Simplificada . . . . . . . . . . . . . . . . 11

2.4.4 Estimação do Número de Grupos . . . . . . . . . . . . . . . . . . 11

3 Descoberta de Conhecimento Distribuído 13

3.1 Agrupamento de Dados Distribuídos . . . . . . . . . . . . . . . . 15

3.1.1 Agregação de Agrupamentos . . . . . . . . . . . . . . . . . . . . . . . . 16

3.1 .2 Agrupamento Paralelo . . . . . . . . . . . . . . . . . . . . . . . . . . . . . . . . 17

3.1.3 Considerações Finais . . . . . . . . . . . . . . . . . . . 17

4 Algoritmos para Agrupamento Fuzzy Colaborativo 19

4.1 Formalização do Problema . . . . . . . . . . . . . . . . . . 20

4.2 Algoritmo CFC — Nível de Interação Fixo . . . . . . . . . . . . . . . . . 21

4.3 Algoritmos Propostos para Agrupamento Colaborativo . . . . . . . . . . . 25

4.3.1 Algoritmos CFC- $\beta_{d}$ e CFC- $\beta_{f}-$ Nível de Interação Variável . . . . . 25

4.3.2 Algoritmos CFCM- $c^{*}$ e CFCM- $\beta_{f}-c^{*}$ - Número de Grupos Variável . . 27

4.3.3 Algoritmo PFCM- $c^{*}$ — Número de Grupos Variável . . . . . . . . . . 33 
4.4 Seleção dos Algoritmos . . . . . . . . . . . . . . . . . 33

5 Avaliação Experimental $\quad 37$

5.1 Conjuntos de Dados . . . . . . . . . . . . . . . . . . . . 37

5.2 Análise dos Algoritmos CFC, CFC- $\beta_{d}$ e CFC- $\beta_{f} \ldots \ldots \ldots$. . . . . . . 39

5.2.1 Nível de Interação $\beta$ Estimado Iterativamente — Algoritmo CFC . . . 39

5.2.2 Nível de Interação $\beta$ Estimado Automaticamente - Algoritmos CFC$\beta_{d}$ e CFC- $\beta_{f} \ldots \ldots \ldots \ldots \ldots \ldots$

5.2.3 Colaboração com Mudanças na Estrutura dos Dados - Algoritmos CFC, CFC- $\beta_{d}$ e CFC- $\beta_{f} \ldots \ldots \ldots$. . . . . . . . . . . 49

5.3 Exemplos Ilustrativos — Cenários de Aplicação . . . . . . . . . . . . . . . . 52

5.3.1 Experimentos com Dados Bi-Dimensionais Provenientes de Diferentes Populações . . . . . . . . . . . . . . . . . . 52

5.3.2 Experimentos com Dados Bi-Dimensionais Provenientes de uma Mesma População . . . . . . . . . . . . . . . . . . 58

5.3.3 Experimentos Envolvendo Sítios com Dados Completamente Aleatórios 60

5.3.4 Experimentos Envolvendo Dados Multivariados . . . . . . . . . . . . . 61

5.3.4.1 Conjunto de Dados Sintéticos . . . . . . . . . . . . 61

5.3.4.2 Conjunto de Dados Reais — Wisconsin Breast Cancer . . . . 64

5.4 Considerações Finais . . . . . . . . . . . . . . . . . . . . . . . . . 64

6 Conclusão e Trabalhos Futuros 67

6.1 Principais Contribuições . . . . . . . . . . . . . . . . 67

6.2 Conclusões . . . . . . . . . . . . . . . . . . . . 68

6.3 Trabalhos Futuros . . . . . . . . . . . . . . . . . . . . 69

$\begin{array}{ll}\text { Referências } & 77\end{array}$ 


\section{Lista de Figuras}

3.1 Exemplo de distribuição homogênea dos dados. O sítio (a) e o sítio (b) contém os registros de clientes de agências bancárias localizadas em diferentes cidades.

3.2 Exemplo de distribuição heterogênea dos dados. O sítio (a) e o sítio (b) contém, respectivamente, dados relativos às condições meteorológicas e à demografia das mesmas cidades. Neste caso o campo "Cidade" é um identificador que relaciona (a) com (b) e vice-versa. . . . . . . . . . . . . . . . . 15

4.1 Matrizes de partição equivalentes para o conceito de matriz de proximidade. . . 25

4.2 Agrupamento colaborativo ocorrendo no sítio de dados $D[i i]$. Todos os outros sítios comunicam seus protótipos e seus respectivos pesos a esse sítio. Como resultado, após o $t$-ésimo estágio de colaboração, tem-se um conjunto de protótipos, $\mathbf{v}_{i}^{t}[i i]$, e seus respectivos pesos, $n_{i}^{t}[i i]\left(i=1,2, \ldots, c^{t}[i i]\right)$, os quais podem ser comunicados a todos os outros sítios no estágio de colaboração $t+1$. Esse mesmo esquema também ocorre nos sítios $D[1], D[2], \ldots, D[i i-1], D[i i+1]$,

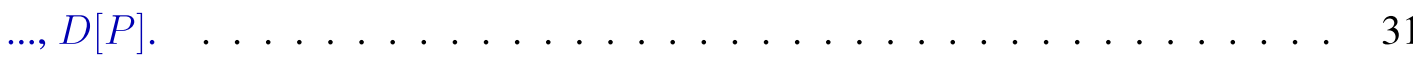

4.3 Árvore de decisão para a escolha dos algoritmos. . . . . . . . . . . . . 35

5.1 Valores de $W_{F}$ de acordo com $\beta$ informado ao algoritmo CFC - Conjunto Sin1. 40

5.2 Comportamento de $W$ - Algoritmo CFC $(\beta=0,25)$ - Conjunto Sin1 . . . . 41

5.3 Valores de $W_{F}$ de acordo $\operatorname{com} \beta$ informado ao algoritmo CFC - Conjunto Sin2. 42

5.4 Comportamento de $W$ - Algoritmo CFC $(\beta=0,95)$ - Conjunto Sin2 . . . . 42

5.5 Grupos obtidos com o algoritmo CFC - Conjunto Sin2. . . . . . . . . . . . 42

5.6 Comportamento de $W$ - Algoritmo CFC- $\beta_{d}-$ Conjunto Sin1. . . . . . . . . 43

5.7 Monitoramento do processo de colaboração no sítio $D[1] \ldots$. . . . . . . . . 45

5.8 Monitoramento do processo de colaboração no sítio $D[2] \ldots \ldots$. . . . . . . . 45

5.9 Monitoramento do processo de colaboração no sítio $D[3] \ldots \ldots$. . . . . . . . 45

5.10 Monitoramento do processo de colaboração no sítio $D[4] \ldots \ldots$. . . . . . . 46

5.11 Monitoramento do processo de colaboração no sítio $D[5] \ldots$. . . . . . . . . . 46

5.12 Comportamento de $W$ - Algoritmo CFC- $\beta_{f}$ - Conjunto Sin1 . . . . . . . . . 48

5.13 Comportamento de $W-$ Algoritmo CFC- $\beta_{f}-$ Conjunto Sin2 . . . . . . . . . 49 
5.14 Grupos obtidos com o algoritmo CFC- $\beta_{f}$ - Conjunto Sin2 . . . . . . . . . . 49

5.15 Sítios de dados do conjunto Sin3 . . . . . . . . . . . . . . . 50

5.16 As posições dos protótipos iniciais e finais para o sítio $D[1]$ em todos os instantes de tempo permaneceram muito próximas das posições ilustradas nesta figura . . . . . . . . . . . . . . . . . . 51

5.17 Grupos do sítio de dados $D[2]_{0}$ - Instante $1 . \ldots \ldots \ldots \ldots$

5.18 Grupos do sítio de dados $D[2]_{5}$ - Instante $6 \ldots \ldots \ldots$. . . . . . 52

5.19 Grupos obtidos em cada sítio pelo algoritmo FCM-c* - Conjunto Sin4. . . . . 53

5.20 Grupos obtidos pelo algoritmo FCM- $c^{*}$ — Conjunto $\operatorname{Sin} 4(D[1] \cup D[2]) \ldots$. . 53

5.21 Grupos obtidos pelo algoritmo CFC- $\beta_{f}$ - Conjunto Sin4. . . . . . . . . . . 54

5.22 Grupos obtidos em cada sítio pelo algoritmo FCM- $c^{*}$ - Conjunto Sin5. . . . . 55

5.23 Grupos obtidos pelo algoritmo FCM- $c^{*}$ — Conjunto $\operatorname{Sin} 5(D[1] \cup D[2]) \ldots \ldots$

5.24 Grupos obtidos pelo algoritmo CFC — Conjunto Sin5 . . . . . . . . . . 57

5.25 Grupos obtidos pelo algoritmo CFC- $\beta_{f}$ - Conjunto Sin5. . . . . . . . . . . 57

5.26 Grupos obtidos pelo algoritmo CFCM- $\beta_{f}-c^{*}-$ Conjunto Sin5 . . . . . . . . . 57

5.27 Grupos obtidos pelo algoritmo PFCM- $c^{*}$ - Conjunto Sin4 . . . . . . . . . . . 58

5.28 Grupos obtidos pelo algoritmo PFCM- $c^{*}$ — Conjunto Sin5 . . . . . . . . . . . 59

5.29 Grupos obtidos pelo algoritmo CFCM- $c^{*}$ - Conjunto Sin4. . . . . . . . . . . 59

5.30 Grupos obtidos pelo algoritmo CFCM-c* — Conjunto Sin5. . . . . . . . . . . 59

5.31 Grupos obtidos pelo CFCM-c* - Conjunto Sin $4-D[2]$ sem estrutura de grupos. 60

5.32 Grupos obtidos pelo CFCM- $\beta_{f}-c^{*}$ - Conjunto Sin $4-D[2]$ sem estrutura de grupos. ................................ 61 


\section{Lista de Tabelas}

4.1 Complexidade de tempo dos algoritmos considerando o mesmo número de objetos $(N)$ e grupos $(c)$ para todos os sítios de dados $-n_{t}$ é o número de iterações, $t$ o número de estágios de colaboração e $n_{p}$ o número de diferentes partições geradas. . . . . . . . . . . . . . . . . . . . 36

4.2 Complexidade de espaço dos algoritmos considerando um único sítio de dados com $N$ objetos e $c$ grupos. . . . . . . . . . . . . . . . . . . 36

4.3 Complexidade de comunicação dos algoritmos considerando o mesmo número de objetos $(N)$ e grupos $(c)$ para todos os sítios de dados $-n_{t}$ é o número de iterações, $t$ o número de estágios de colaboração e $n_{p}$ o número de diferentes partições geradas. . . . . . . . . . . . . . . . . . . 36

5.1 Protótipos obtidos com o algoritmo CFC $(\beta=0,25)$ - Conjunto Sin1 . . . . . 40

5.2 Protótipos obtidos com o algoritmo CFC- $\beta_{d}-$ Conjunto Sin1. . . . . . . . . 44

5.3 Protótipos obtidos com o algoritmo CFC- $\beta_{f}$ - Conjunto Sin1 . . . . . . . . . . 47

5.4 Valores de $\beta[i i \mid j j]$ para todos os pares de sítios — Conjunto Sin1. . . . . . . 48

5.5 Vetores médios representantes dos grupos em $D[1]$ e $D[2]$ - Conjunto Sin4-8. 62

5.6 Protótipos de grupos obtidos pelo algoritmo CFCM- $\beta_{f}-c^{*}$ - Conjunto Sin4-8. 62

5.7 Protótipos de grupos obtidos pelo algoritmo CFCM- $c^{*}$ - Conjunto Sin4-8. . . 62

5.8 Vetores médios representantes dos grupos em $D[1]$ e $D[2]$ - Conjunto Sin5-4. 63

5.9 Protótipos de grupos obtidos pelo algoritmo FCM-c* $-\operatorname{Sin} 5-4(D[1] \cup D[2])$. . 63

5.10 Protótipos de grupos obtidos pelo algoritmo CFCM- $\beta_{f}-c^{*}$ - Conjunto Sin5-4. 63

5.11 Protótipos de grupos obtidos pelo algoritmo PFCM-c* - Conjunto Sin5-4. . . 63 


\section{Lista de Abreviaturas}

CFC: $\quad$ Collaborative Fuzzy Clustering.

CFC- $\beta_{d}$ :

CFC com $\beta$ estimado em cada estágio de colaboração.

$\mathrm{CFC}-\beta_{f}$ :

CFC com $\beta$ estimado uma única vez.

CFCM:

Collaborative Fuzzy C-Means.

CFCM- $c^{*}$ :

CFCM com número de grupos c estimado automaticamente.

CFCM- $\beta_{f}-c^{*}$ : $\quad$ CFCM com número de grupos $c$ e $\beta$ estimados automaticamente.

DDC:

Distributed Data Clustering.

DDM:

Distributed Data Mining.

DKD:

Distributed Knowledge Discovery.

DM:

Data Mining.

FCM:

Fuzzy C-Means.

FCM- $c^{*}$ :

Ordered Multiple Runs of FCM.

KDD:

Knowledge Discovery in Databases.

PFCM:

Parallel Fuzzy C-Means.

PFCM- $c^{*}$ :

PFCM com número de grupos c estimado automaticamente.

SC:

Silhueta Crisp.

SF:

Silhueta Fuzzy.

SFS:

Silhueta Fuzzy Simplificada. 


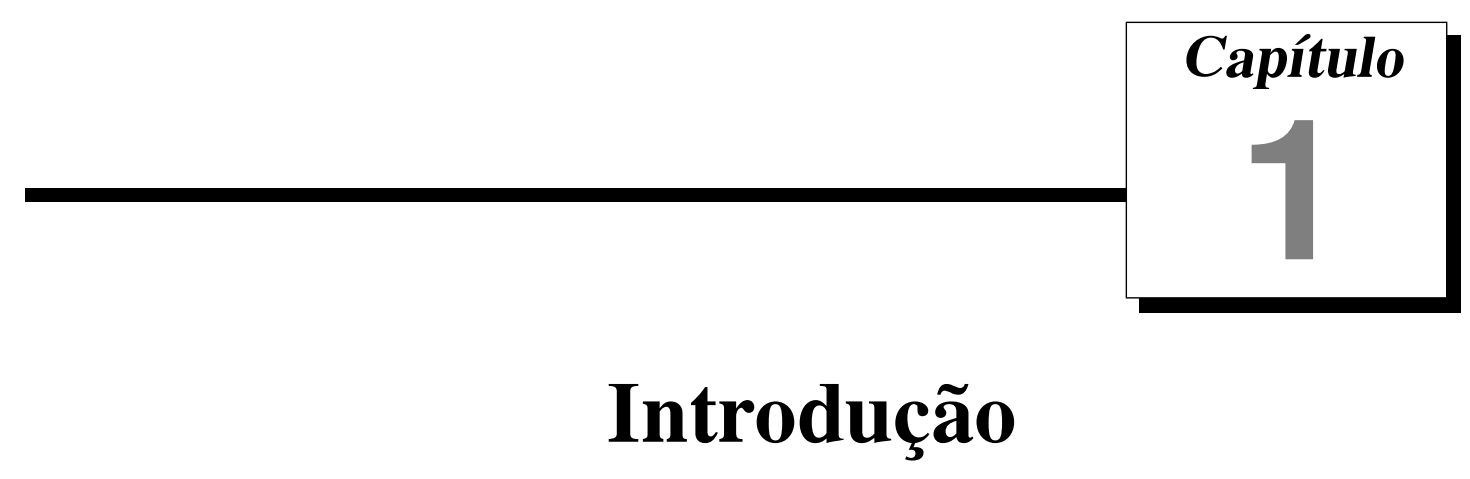

A contínua evolução da tecnologia computacional tem nos possibilitado cada vez mais coletar, gerar e processar dados. Como consequência e aliada ao crescente aumento da capacidade de armazenamento, tem se tornado comum a utilização de grandes bancos de dados em diversas áreas da atividade humana. Contudo, esses avanços tecnológicos têm causado um problema conhecido como superabundância de dados, pois nossas capacidades de coletar e armazenar dados têm superado nossa habilidade de analisar e extrair conhecimento dos mesmos (Fayyad et al., 1996b). Nesse sentido, se faz necessária a aplicação de técnicas e ferramentas que sejam capazes de extrair, dos dados disponíveis, de forma inteligente e automática, as informações úteis que representem conhecimento. No entanto, tal procedimento, apesar de muito interessante, e até intuitivo, é tecnicamente difícil e desafiador (Brachman \& Anand, 1996), envolvendo diversas áreas do conhecimento humano e, sobretudo, a participação ativa do próprio ser humano.

Com o objetivo de utilizar bancos de dados de maneira inteligente, surgiu o termo mineração de dados, ou Data Mining (DM), (Han \& Kamber, 2006; Tan et al., 2006; Witten \& Frank, 2005; Fayyad et al., 1996c; Bigus, 1996; Chen et al., 1996) que, ao próprio sentido da palavra, significa o processo de exploração de grandes bancos de dados (minas de ouro) a fim de extrair algum conhecimento (ouro), recuperando as informações valiosas e modelos que antes estavam ocultos devido à superabundância de dados. Formalmente, DM se constitui de alguns passos de um processo mais amplo denominado descoberta de conhecimento em bancos de dados, ou Knowledge Discovery in Databases (KDD). Esse termo pode ser definido como sendo o processo não trivial de identificação de padrões válidos, novos, potencialmente úteis e compreensíveis em grandes bancos de dados (Fayyad et al., 1996c). De forma mais abrangente, KDD refere-se ao processo interativo e iterativo de descoberta de conhecimento em conjuntos de dados, incorporando conhecimento de domínio e interpretação de resultados, com ênfase na aplicação dos métodos de DM (Fayyad et al., 1996c). Assim, o processo de KDD pode ser divi- 
dido em três grandes etapas (Tan et al., 2006): (i) a preparação dos dados (seleção, purificação e pré-processamento); (ii) a aplicação de um algoritmo de DM; e (iii) a análise das informações, objetivando-se a avaliação criteriosa do conhecimento obtido. De acordo com (Han \& Kamber, 2006; Klösgen \& Zytkow, 1996; Piatetsky-Shapiro \& Frawley, 1991), KDD é útil em vários campos da ciência, bem como em várias aplicações práticas. Como exemplo, podem-se citar aplicações em bioinformática, marketing, medicina, engenharia, finanças e astronomia. Além disso, nos últimos anos o processo de KDD, e consequentemente a tarefa de DM, têm encontrado espaço em um novo e complexo domínio que é fruto dos avanços das tecnologias das redes e da internet, envolvendo, portanto, computação distribuída (Park \& Kargupta, 2002; Park et al., 2002). Em resumo, esses avanços são guiados pela necessidade de estreitamento das relações entre diversos setores da sociedade. Nesse sentido, a globalização, o business-to-commerce e a colaboração on-line entre as organizações têm aumentado a necessidade de mineração de dados interorganizacionais, o que envolve a análise de dados que estão fisicamente distribuídos (Datta et al., 2006; Tsoumakas, 2004). Essa tendência ocasionou o surgimento de um novo campo de estudo, como uma extensão do processo de KDD, denominado de descoberta de conhecimento distribuído, ou Distributed Knowledge Discovery (DKD) (Zhang et al., 2004; Kargupta et al., 2001; Johnson \& Kargupta, 2000). Esse novo campo de estudo abrange técnicas e métodos para minerar dados que estão fisicamente distribuídos, lidando com as adversidades impostas por este domínio (e.g., restrições de segurança, privacidade, largura de banda, etc.) (Kargupta et al., 2001). Em particular, DKD envolve métodos e algoritmos para agrupamento de dados distribuídos, ou Distributed Data Clustering (DDC) (Silva \& Klusch, 2006; Merugu \& Ghosh, 2005; Klusch et al., 2003), os quais têm como objetivo agrupar dados que estão distribuídos em diferentes subconjuntos (sítios de dados). Como exemplo desses métodos e algoritmos podemse citar aqueles baseados na agregação de agrupamentos, ou cluster ensembles (Tumer \& Agogino, 2008; Topchy et al., 2004; Strehl \& Ghosh, 2002; Johnson \& Kargupta, 2000), os quais visam obter um modelo global dos dados por meio da agregação de diversos agrupamentos (modelos locais) obtidos em diferentes sítios de dados. Alguns outros algoritmos para agrupamento de dados distribuídos têm sido desenvolvidos e estudados (Kargupta \& Chan, 2000). Em particular, algumas variantes do algoritmo amplamente conhecido Fuzzy C-Means (FCM) (Bezdek, 1981; Dunn, 1973), as quais têm sido investigadas sob o nome de agrupamento fuzzy colaborativo (Pedrycz \& Rai, 2008; Pedrycz, 2007, 2002).

Agrupamento colaborativo ocorre quando algoritmos de agrupamento aplicados em diferentes sítios de dados comunicam ativamente descobertas entre si (Pedrycz \& Rai, 2008). Esse processo tem como objetivo revelar estruturas de grupos que são (aproximadamente) comuns em diferentes sítios de dados (Pedrycz, 2002). Em (Rahimi et al., 2004) foi proposto o algoritmo Parallel Fuzzy C-Means (PFCM), como uma extensão do algoritmo FCM que, além de ser capaz de atuar em domínios onde os dados estão distribuídos, tem se mostrado potencialmente útil para agrupamento colaborativo. Pedrycz \& Rai (2008), por sua vez, introduziram um algoritmo, aqui chamado de Collaborative Fuzzy Clustering (CFC), que visa agrupar dados distribuídos baseando-se no conceito de colaboração idealizado em (Pedrycz, 2002). Esses 
trabalhos se constituíram no ponto de partida desta pesquisa, a qual foi motivada por algumas limitações encontradas nos algoritmos PFCM e CFC. De maneira mais específica, esses algoritmos dependem de alguns parâmetros que devem ser fornecidos pelo usuário, tal como o número de grupos $(c)$ a serem identificados e, em particular, para o algoritmo $\mathrm{CFC}$, o nível de interação $(\beta)$. Muito comumente, os valores ótimos desses parâmetros não são conhecidos $a$ priori pelo usuário ou o especialista de domínio, o que torna o uso desses algoritmos, de certa forma, restrito. Com o objetivo de superar essas limitações, estimando-se automaticamente (a partir dos dados) os valores desses parâmetros, cinco novos algoritmos foram desenvolvidos nesse trabalho. Esses algoritmos, bem como os algoritmos PFCM e CFC, foram estudados em dois cenários específicos de aplicação que levam em conta duas suposições sobre os dados (i.e., se os dados são de uma mesma ou de diferentes populações). Na prática, assumindo-se essas suposições, bem como a dificuldade em se definir alguns dos parâmetros (que possam ser requeridos), alguns algoritmos podem ser mais vantajosos do que outros. A partir desse ponto de vista, o estudo realizado nessa pesquisa busca auxiliar o usuário interessado em escolher, dentre os algoritmos disponíveis, o mais apropriado para o problema que se tem em mãos. Exemplos ilustrativos destacam as diferenças de desempenho entre esses algoritmos, permitindo derivar algumas conclusões que podem ser úteis ao aplicar agrupamento colaborativo na prática.

\subsection{Objetivos}

Os principais objetivos desse trabalho foram:

- O desenvolvimento de novos algoritmos para agrupamento fuzzy colaborativo que superem algumas das limitações existentes nos algoritmos PFCM (Rahimi et al., 2004) e CFC (Pedrycz \& Rai, 2008).

- O estudo de algoritmos fuzzy colaborativo em cenários específicos de aplicação, com o objetivo de identificar os mais vantajosos levando-se em conta tanto as suposições sobre os dados quanto a dificuldade em se definir parâmetros que possam ser requeridos.

\subsection{Organização da Dissertação}

Este documento está organizado da seguinte maneira:

Capítulo 2 - Agrupamento de Dados: Nesse capítulo é apresentada uma revisão bibliográfica sucinta sobre agrupamento de dados. Em particular, são descritos dois algoritmos para agrupamento amplamente conhecidos e importantes para este trabalho. Além disso, são abordadas formas de se validar agrupamentos e estimar o número correto de grupos a partir de índices de validação.

Capítulo 3 - Descoberta de Conhecimento Distribuído: Nesse capítulo são apresentados os principais conceitos sobre agrupamento de dados distribuídos. Em particular, são apre- 
sentados alguns métodos que podem ser usados em domínios distribuídos, tais como a agregação de agrupamentos e agrupamento paralelo.

Capítulo 4 - Algoritmos para Agrupamento Fuzzy Colaborativo: Nesse capítulo são apresentados os principais conceitos sobre agrupamento de dados fuzzy colaborativo. Em particular, são descritos dois algoritmos que atuam de forma colaborativa (PFCM e CFC). Além disso, são introduzidos os cinco novos algoritmos desenvolvidos nesse trabalho.

Capítulo 5 - Avaliação Experimental: Nesse capítulo são apresentados os experimentos realizados nesse trabalho. Tais experimentos têm como objetivo fornecer exemplos ilustrativos que destacam as diferenças de desempenho entre os algoritmos abordados e desenvolvidos durante esta pesquisa.

Capítulo 6 - Conclusão e Trabalhos Futuros: Nesse capítulo é apresentada a conclusão do trabalho, descrevendo-se também as principais contribuições e algumas sugestões para trabalhos futuros. 


\section{Capítulo}

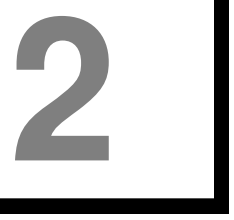

\section{Agrupamento de Dados}

A atividade de categorizar objetos em grupos tem uma longa história, sendo considerada uma das tarefas mais primitivas do ser humano (Jain, 2010; Everitt et al., 2001; Hansen \& Jaumard, 1997). De fato, formar grupos de objetos similares é muito comum e natural para o ser humano. Por exemplo, pessoas podem agrupar plantas de acordo com sua espécie, animais de acordo com sua raça, cidades de acordo com sua geografia, etc. No entanto, é importante enfatizar que a concepção de grupos é subjetiva, i.e., pessoas diferentes podem formar grupos diferentes com o mesmo conjunto de objetos. De forma mais pragmática, o processo de agrupamento de dados (clustering) consiste na identificação de um conjunto de categorias - também chamadas de grupos ou de clusters - que descrevam um conjunto de dados (Fayyad et al., 1996b). Essa tarefa usualmente envolve maximizar a homogeneidade entre os objetos de um mesmo grupo e a heterogeneidade entre objetos de grupos distintos. O estudo formal de algoritmos e métodos para agrupar dados compreende a análise de agrupamento de dados, a qual tem ampla aplicação em Data Mining (DM) (Han \& Kamber, 2006; Chen et al., 1996) e várias áreas do conhecimento humano (Xu \& Wunsch, 2009; Jain \& Dubes, 1988a), tais como bioinformática, redes de sensores e segurança computacional. Além disso, de acordo com Everitt et al. (2001); Hartigan (1975); Anderberg (1973), o conceito de agrupamento de dados também está presente em grandes campos da ciência, tais como: engenharia, economia, medicina, astronomia, ciências sociais e ciências da computação. Devido a essa ampla aplicação prática, há uma vasta e crescente literatura sobre esse assunto. Estudos detalhados sobre métodos e algoritmos para agrupamento de dados podem ser encontrados nos livros de Xu \& Wunsch (2009); Everitt et al. (2001); Kaufman \& Rousseeuw (1990); Jain \& Dubes (1988a); Hartigan (1975). No entanto, mesmo com o notável empenho das pesquisas nessa área nas últimas décadas, não existe um algoritmo de agrupamento universal, i.e., que resolva todos os problemas. Normalmente os algoritmos são projetados de acordo com suposições sobre as formas dos grupos ou a distribuição dos dados e, inevitavelmente, favorecem algum tipo de bias (Xu \& Wunsch, 2009). 
De maneira mais abrangente, métodos e algoritmos para agrupamento de dados são capazes de explorar e identificar estruturas presentes nos dados, obtendo-se grupos de objetos similares. Em outras palavras, algoritmos para agrupamento de dados visam descobrir os grupos naturais presentes nos dados sem o conhecimento prévio sobre as suas classes ou categorias (Jain, 2010). Nesse sentido, agrupamento de dados corresponde ao paradigma de aprendizado não supervisionado, no qual os dados disponíveis não estão rotulados (Bishop, 2007; Mitchell, 1997).

Os algoritmos para agrupamento de dados podem ser divididos de diferentes formas. Uma taxonomia muito comum e interessante para o presente trabalho envolve categorizar os algoritmos em (Tan et al., 2006):

Algoritmo particional versus hierárquico: Algoritmos particionais produzem uma única partição ou agrupamento (conjunto de grupos) dos dados de acordo com um número específico de grupos, enquanto que os algoritmos hierárquicos produzem uma estrutura de partições sequencialmente aninhadas, respeitando uma hierarquia.

Grupos disjuntos versus sobrepostos: Uma partição com grupos disjuntos (exclusivos) é uma partição na qual cada objeto pertence exclusivamente a um único grupo. Esse tipo de partição é também chamada de partição rígida, hard ou crisp. Por outro lado, uma partição com grupos sobrepostos (não exclusivos) é uma partição na qual cada objeto pode pertencer a mais de um grupo (e.g., partições fuzzy).

Diversos algoritmos para agrupamento particional têm sido propostos nas últimas décadas, a maioria deles baseados na: minimização das somas do erro quadrático (e.g., k-means (MacQueen, 1967)); estatística (e.g., Expectation-Maximization (EM) (Dempster et al., 1977)); teoria dos grafos (e.g., CLICK (Sharan \& Shamir, 2000)); e lógica fuzzy (e.g., Fuzzy C-Means (FCM) (Bezdek, 1981)). Em particular, o presente trabalho envolve algoritmos para agrupamento particional dos dados capazes de lidar com grupos sobrepostos. Especificamente, esses algoritmos geram partições fuzzy (Bezdek, 1981; Dunn, 1973) e geralmente utilizam a medida de distância Euclidiana quadrada para calcular a dissimilaridade entre objetos. De maneira mais formal, em problemas de agrupamento, os dados a serem explorados consistem de um conjunto $X$ de $N$ objetos não rotulados (alternativamente chamados de tuplas, registros ou exemplos), $X=\left\{\mathbf{x}_{1}, \mathbf{x}_{2}, \ldots, \mathbf{x}_{N}\right\}$, onde cada $\mathbf{x}_{j}=\left[x_{j 1}, \ldots, x_{j d}\right]$ é um vetor de valores de um conjunto de $d$ atributos (alternativamente chamados de características ou variáveis). Dado um objeto $\mathbf{x}_{j} \in X$ e um ponto qualquer $\mathbf{v}_{i}=\left[v_{i 1}, \ldots, v_{i d}\right]$, a distância Euclidiana quadrada entre $\mathbf{x}_{j}$ e $\mathbf{v}_{i}$ é dada pela Eq. (2.1):

$$
\left\|\mathbf{x}_{j}-\mathbf{v}_{i}\right\|^{2}=\sum_{t=1}^{d}\left(x_{j t}-v_{i t}\right)^{2}
$$




\subsection{Complexidade do Problema}

O problema a ser resolvido por algoritmos de agrupamento que produzem partições rígidas (como um caso particular de algoritmos para agrupamento fuzzy) pode ser definido formalmente como a divisão do conjunto $X$ de $N$ objetos, $X=\left\{\mathbf{x}_{1}, \mathbf{x}_{2}, \ldots, \mathbf{x}_{N}\right\}$, em $c$ grupos disjuntos, $C=\left\{C_{1}, C_{2}, \ldots, C_{c}\right\}$ tal que $C_{1} \cup C_{2} \cup \ldots \cup C_{c}=X, C_{p} \neq \phi$ e $C_{p} \cap C_{q}=\phi$ para $p \neq q$. De acordo com (Liu, 1968), sendo conhecido o número de grupos $c$, o número de maneiras $M$ de se agrupar $N$ objetos em $c$ grupos é dado pela Eq. (2.2):

$$
M(N, c)=\frac{1}{c !} \sum_{i=0}^{c}(-1)^{i}\left(\begin{array}{l}
c \\
i
\end{array}\right)(c-i)^{N}
$$

A título de ilustração, a Eq. (2.2), no problema de agrupar 30 objetos em 3 grupos oferece aproximadamente $2 \times 10^{14}$ possíveis soluções (agrupamentos) (Xu \& Wunsch, 2009). Claramente pode-se concluir que a utilização de métodos de força bruta para encontrar o melhor agrupamento é inviável na prática. Além disso, deve-se considerar que muito comumente problemas de agrupamento de dados lidam com centenas e até milhares de objetos. Por isso, métodos baseados em heurísticas são normalmente usados, almejando soluções aproximadas.

\subsection{Algoritmo K-Means}

O algoritmo k-means (Jain, 2010; MacQueen, 1967) é um dos mais simples e conhecidos algoritmos para agrupamento particional, tendo como objetivo minimizar o erro quadrático (soma das distâncias médias entre os centróides e objetos dos grupos correspondentes). Nesse algoritmo, os centróides são os pontos médios de cada grupo que são recalculados a cada iteração. Em sua versão original, o $k$-means produz uma partição com $k$ grupos hiper-esféricos (globulares) utilizando-se da medida de distância Euclidiana quadrada - Eq. (2.1). Os principais passos do algoritmo $k$-means estão sumarizados no Algoritmo 1.

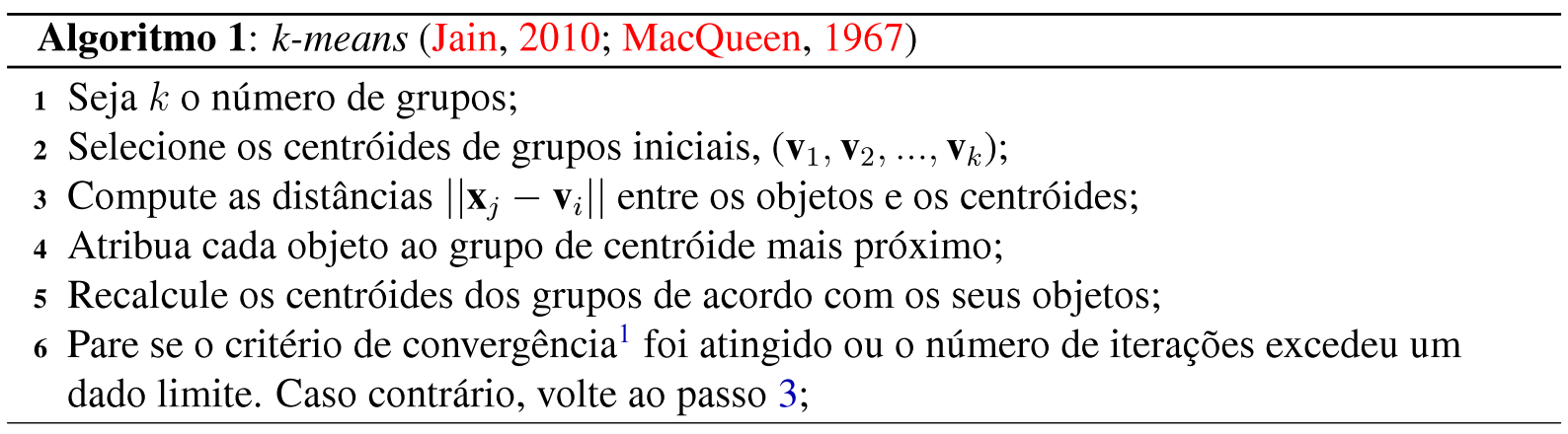

A complexidade do algoritmo $k$-means é estimada em $O\left(k \cdot N \cdot d \cdot n_{t}\right)$, onde $k$ é o número de grupos, $N$ é o número de objetos, $d$ é o número de atributos e $n_{t}$ o número de iterações.

\footnotetext{
${ }^{1}$ Um critério de convergência normalmente usado é quando os centróides não se alterarem significativamente em duas iterações consecutivas.
} 
Adicionalmente, o k-means é simples de implementar e pode atingir resultados satisfatórios com poucas iterações (Jain, 2010; Jain et al., 1999). No entanto, esse algoritmo é sensível à escolha dos centróides iniciais e dependente da informação do número de grupos $(k)$, o qual deve ser fornecido pelo usuário. Além disso, o k-means produz bons resultados apenas quando não há sobreposição de grupos, i.e., quando os grupos são disjuntos (compactos e bem separados). Para tentar superar essas limitações, diversas variantes do $k$-means têm sido desenvolvidas (Jain, 2010; Steinbach et al., 2000; Kaufmann \& Rousseeuw, 1987; Symon, 1977). Em particular, uma variante de especial importância para esta pesquisa foi proposta por Dunn (1973) e, posteriormente, melhorada por Bezdek (1981). Essa extensão do $k$-means é descrita na próxima seção.

\subsection{Algoritmo Fuzzy C-Means}

O algoritmo Fuzzy C-Means (FCM) (Bezdek, 1981; Dunn, 1973) é uma extensão do algoritmo $k$-means para o contexto fuzzy. Enquanto o algoritmo $k$-means assume que cada objeto deve pertencer exclusivamente a um único grupo, o algoritmo FCM permite relaxar essa restrição, possibilitando que cada objeto pertença, sob graus diferentes, a cada um dos grupos. Dessa maneira, esse algoritmo tem como principal vantagem a identificação de grupos sobrepostos. $\mathrm{O}$ algoritmo FCM usa protótipos como representantes dos grupos. Tais protótipos guardam informações análogas àquelas armazenadas pelos centróides computados pelo $k$-means. No entanto, cada protótipo é computado levando-se em conta todos os objetos e suas respectivas pertinências aos grupos. Especificamente, o algoritmo FCM busca, por meio de um procedimento iterativo, minimizar a função objetivo em (2.3):

$$
\begin{gathered}
\min _{u_{i j}, \mathbf{v}_{i}} J=\sum_{j=1}^{N} \sum_{i=1}^{c} u_{i j}^{m}\left\|\mathbf{x}_{j}-\mathbf{v}_{i}\right\|^{2} \\
\text { s.a. }\left\{\begin{array}{c}
0 \leq u_{i j} \leq 1 \\
\sum_{i=1}^{c} u_{i j}=1 \quad \forall j \epsilon\{1, \ldots, N\} \\
0<\sum_{j=1}^{N} u_{i j}<N \quad \forall i \epsilon\{1, \ldots, c\}
\end{array}\right.
\end{gathered}
$$

onde $\mathbf{x}_{j} \in \Re^{d}(j=1, \ldots, N)$ são os objetos a serem agrupados em $c$ grupos, $\mathbf{v}_{i} \in \Re^{d}(i=1, \ldots, c)$ são os protótipos dos grupos, $u_{i j}$ representa a pertinência do $j$-ésimo objeto ao $i$-ésimo grupo e, por fim, $m \in] 1, \infty[$ é um parâmetro de ponderação que controla o quão fuzzy é uma partição. Usualmente, bem como nesse trabalho, adota-se $m=2$.

Os principais passos do FCM estão sumarizados no Algoritmo 2, o qual é usado para minimizar $J$ em (2.3). Similarmente ao seu predecessor $k$-means, o algoritmo FCM é considerado rápido e simples. A sua complexidade é estimada em $O\left(c^{2} \cdot N \cdot d \cdot n_{t}\right)$, onde $c$ é o número de grupos, $N$ é o número de objetos, $d$ é o número de atributos e $n_{t}$ o número de iterações. Entretanto, assim como o k-means, o FCM é sensível à escolha dos protótipos iniciais, além 
de depender da informação do número de grupos $(c)$. A próxima seção aborda alguns métodos para tentar superar essas dificuldades.

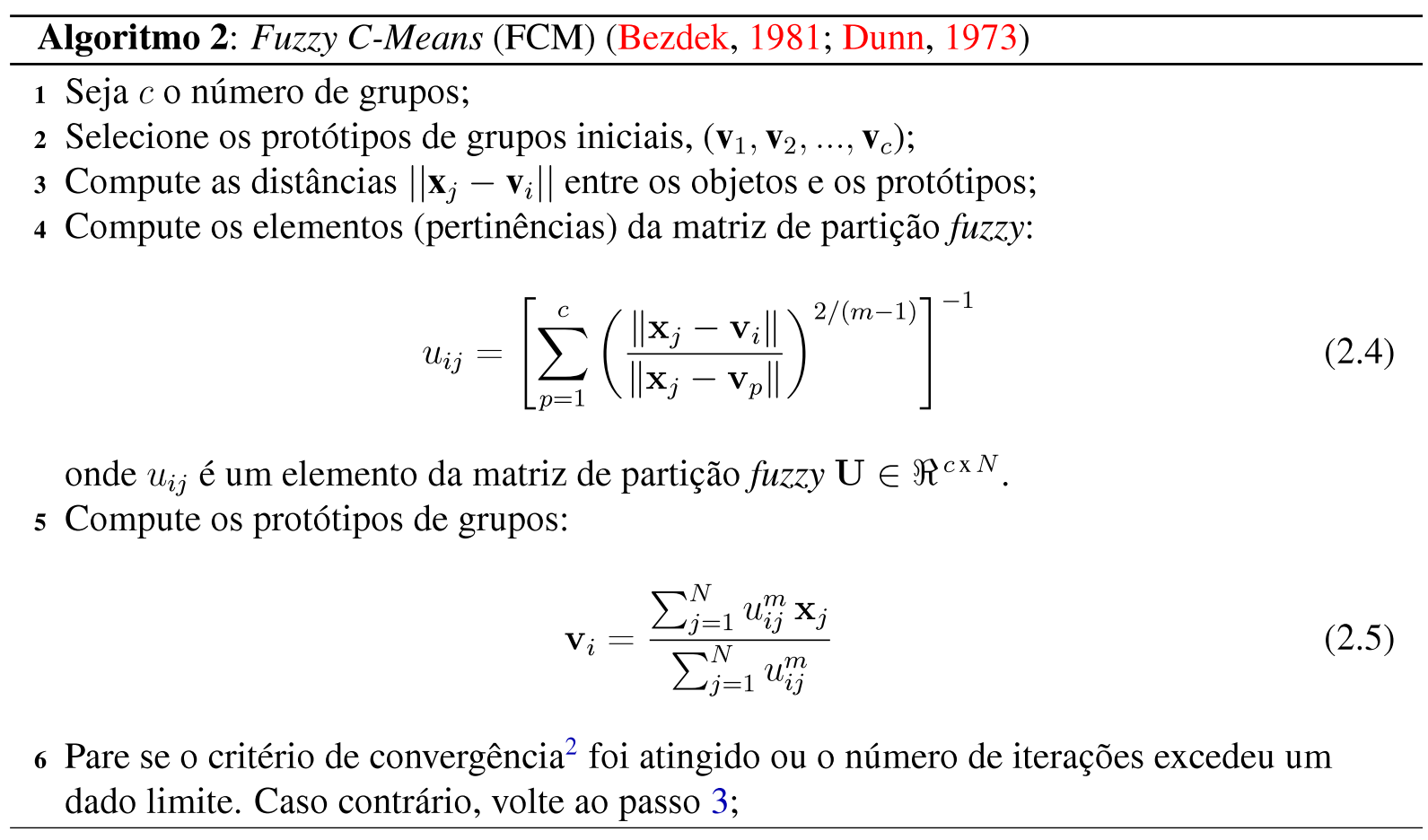

\subsection{Validação de Agrupamentos de Dados}

Conforme contextualizado nas Seções 2.2 e 2.3, os algoritmos para agrupamento particional k-means e Fuzzy C-Means (FCM) requerem como parâmetro de entrada o número de grupos a serem identificados (Kaufman \& Rousseeuw, 1990; Milligan, 1996). Adicionalmente, tais algoritmos também estão sujeitos a ficarem presos em mínimos locais (e.g., por conta de uma má escolha dos protótipos iniciais). Essas dificuldades podem resultar em partições de baixa qualidade e que não representam adequadamente a real estrutura de grupos presente nos dados. Nesse sentido, alguns métodos podem ser usados para medir a qualidade de diferentes soluções obtidas por diferentes configurações de um determinado algoritmo ou mesmo pelo uso de diferentes algoritmos. De maneira mais específica, dado um conjunto de soluções candidatas (partições de dados), para identificar (de forma quantitativa) a melhor delas pode-se usar um critério numérico (Jain \& Dubes, 1988b). Existem diversos critérios numéricos para validar agrupamentos na literatura (Vendramin et al., 2009; Halkidi et al., 2001; Dumitrescu et al., 2000; Höppner et al., 1999) e muitos deles podem ser utilizados para comparar diferentes partições e também estimar o número de grupos (esses são comumente chamados de índices de validade relativos). Em particular, neste trabalho é usado um índice de validade relativo capaz de avaliar partições fuzzy (como as geradas pelo algoritmo FCM). Esse índice, chamado de Silhueta Fuzzy (SF) (Campello \& Hruschka, 2006), pode estimar, dentre um conjunto de partições fuzzy,

\footnotetext{
${ }^{2}$ Como critério de convergência pode-se adotar um limiar $\varepsilon$ para a máxima diferença absoluta entre os elementos da matriz de partição em duas iterações consecutivas. Um valor comum e usado neste trabalho é $\varepsilon=10^{-3}$.
} 
qual delas melhor se ajusta aos dados - mesmo que esse conjunto contenha partições com diferentes números de grupos. Em outras palavras, a SF também é capaz de estimar o número correto de grupos $(c)$. Esse índice é, na realidade, uma extensão do tradicional índice conhecido como silhueta (Kaufman \& Rousseeuw, 1990), aqui denominado Silhueta Crisp (SC), a qual foi originalmente concebida para a avaliação de partições rígidas (tal como as geradas pelo algoritmo $k$-means). Esses dois índices (SC e SF) são abordados mais detalhadamente nas próximas seções.

\subsubsection{Silhueta Crisp}

A Silhueta Crisp (SC) baseia-se na similaridade entre os objetos de um grupo e na dissimilaridade destes objetos em relação ao grupo vizinho mais próximo para avaliar quantitativamente partições de dados (Kaufman \& Rousseeuw, 1990). De maneira mais formal, considere um objeto $j \in\{1,2, \ldots, N\}$ pertencente a um grupo $p \in\{1, \ldots, c\}$. A silhueta deste objeto pode ser calculada pela Eq. (2.6):

$$
s_{j}=\frac{b_{p j}-a_{p j}}{\max \left\{a_{p j}, b_{p j}\right\}}
$$

onde $a_{p j}$ é a distância média do objeto $j$ a todos os objetos de seu grupo $p$. Para calcular $b_{p j}$, considere a distância média $d_{q j}$ do objeto $j$ a todos os objetos de um outro grupo $q$. Pode-se definir $b_{p j}$ como o menor valor $d_{q j}$ calculado para $q \in\{1, \ldots, c\}, q \neq p$, ou seja, $b_{p j}=\min \left(d_{q j}\right)$, $q \neq p$. Dessa forma, o valor de $b_{p j}$ representa a dissimilaridade do objeto $j$ ao grupo vizinho mais próximo. O denominador desta equação é usado apenas como um termo de normalização.

É possível verificar que $-1<s_{j}<1, \log$, quanto maior o valor de $s_{j}$, melhor é a atribuição do objeto $j$ ao grupo $p$. É importante ressaltar que, caso o grupo $p$ seja um singleton, i.e., se ele é constituído de apenas um único objeto $j$, então a silhueta para este objeto é definida como $s_{j}=0$ (Kaufman \& Rousseeuw, 1990). Finalmente, a média dos valores de $s_{j}(j=1,2, \ldots, N)$ é aqui denominada de Silhueta Crisp (SC). Mais formalmente, a SC é computada de acordo com a Eq. (2.7):

$$
S C=\frac{1}{N} \sum_{j=1}^{N} s_{j}
$$

Dado um conjunto de partições, a melhor partição é aquela que maximiza o valor de $S C$ na Eq. (2.7). Convém destacar que a SC é útil e comumente usada para a avaliação de partições rígidas e mostrou resultados positivos em um estudo recente (Vendramin et al., 2010). A seguir, a Silhueta Fuzzy (SF) (Campello \& Hruschka, 2006) é descrita como sendo uma generalização da SC capaz de avaliar partições fuzzy. 


\subsubsection{Silhueta Fuzzy}

A Silhueta Fuzzy (SF) (Campello \& Hruschka, 2006) introduz nos cálculos da SC (Seção 2.4.1) a informação capturada pela matriz de partição, i.e., as pertinências dos objetos aos grupos obtidas pelo algoritmo FCM (Seção 2.3). A incorporação dessas pertinências possibilita revelar regiões de alta densidade, aumentando a importância dos objetos próximos aos protótipos, enquanto reduz a importância de objetos em áreas de sobreposição de grupos. Devido a essa propriedade, a SF é particularmente útil em problemas de agrupamento fuzzy. Formalmente, a Silhueta Fuzzy (SF) é definida pela Eq. (2.8):

$$
S F=\frac{\sum_{j=1}^{N}\left(u_{p j}-u_{q j}\right)^{\alpha} s_{j}}{\sum_{j=1}^{N}\left(u_{p j}-u_{q j}\right)^{\alpha}}
$$

onde $s_{j}$ é a silhueta do objeto $j-$ Eq. (2.6) $-u_{p j}$ e $u_{q j}$ são o primeiro e o segundo maiores elementos da $j$-ésima coluna da matriz de partição, respectivamente, e por fim, $\alpha \geq 0$ é um coeficiente de ponderação, cujo valor normalmente adotado é 1 . Repare que ao usar $\alpha=0$ a SF recai em um caso particular, comportando-se como a SC.

\subsubsection{Silhueta Fuzzy Simplificada}

Os índices de validade relativos descritos nas Seções 2.4.1 e 2.4.2 (SC e SF, respectivamente) dependem da computação de todas as distâncias entre todos os objetos. Esse processo pode tornar a validação de agrupamento pouco eficiente em termos de tempo computacional. No entanto, essa demanda de processamento pode ser reduzida substituindo-se os termos $b_{p j} \mathrm{e}$ $a_{p j}$ na Eq. (2.6) por versões simplificadas baseadas na distância entre objetos e protótipos dos grupos correspondentes (Hruschka et al., 2006). Nesse sentido, na Eq. (2.6), o parâmetro $a_{p j}$ passa a ser a dissimilaridade do objeto $j$ ao protótipo de seu grupo e $b_{p j}$ passa a ser a distância do objeto $j$ ao protótipo do grupo vizinho mais próximo. Essa modificação pode não diminuir significativamente a acurácia e é capaz de reduzir significantemente o tempo computacional de $\mathrm{O}\left(N^{2}\right)$ para $\mathrm{O}(N)$ (Horta et al., 2009). Esse trabalho utiliza essa versão simplificada da SF, a qual passou a ser chamada de Silhueta Fuzzy Simplificada (SFS).

\subsubsection{Estimação do Número de Grupos}

Idealmente, o valor do número de grupos $(c)$ informado ao algoritmo de agrupamento deveria corresponder ao número de grupos naturais dos dados (Jain \& Dubes, 1988a). Dessa forma, a informação de um valor inadequado de $c$ ao algoritmo de agrupamento poderia ocasionar numa distorção da real estrutura de grupos presente nos dados, resultando em sérios problemas de interpretação da partição gerada (Xu \& Wunsch, 2009). Essa é a principal dificuldade enfrentada pelo usuário de algoritmos de agrupamento particionais. No entanto, na prática, é possível superar essa limitação por meio de alguns métodos, os quais normalmente são baseados em um procedimento geral que envolve dois passos: (i) gerar agrupamentos dos dados para diferentes 
números de grupos; (ii) avaliar por meio de um índice de validade relativo — tal como a Silhueta Fuzzy Simplificada (SFS) — a qualidade desses agrupamentos objetivando-se identificar o melhor deles. Esse trabalho utiliza um método baseado nesses dois passos, chamado de Ordered Multiple Runs of FCM (FCM-c*) (Campello et al., 2009), para estimar o número de grupos. O FCM- $c^{*}$ é sumarizado no Algoritmo 3, o qual consiste em executar o algoritmo FCM repetidamente para diferentes números de grupos $(c)$. Para cada valor de $c$, um determinado número de partições geradas são avaliadas por meio de algum índice de validade relativo - e.g., SFS (Seção 2.4.4). A partição de melhor qualidade, juntamente com o valor estimado de grupos $c$ (de acordo com o índice usado), são obtidos como resultado final.

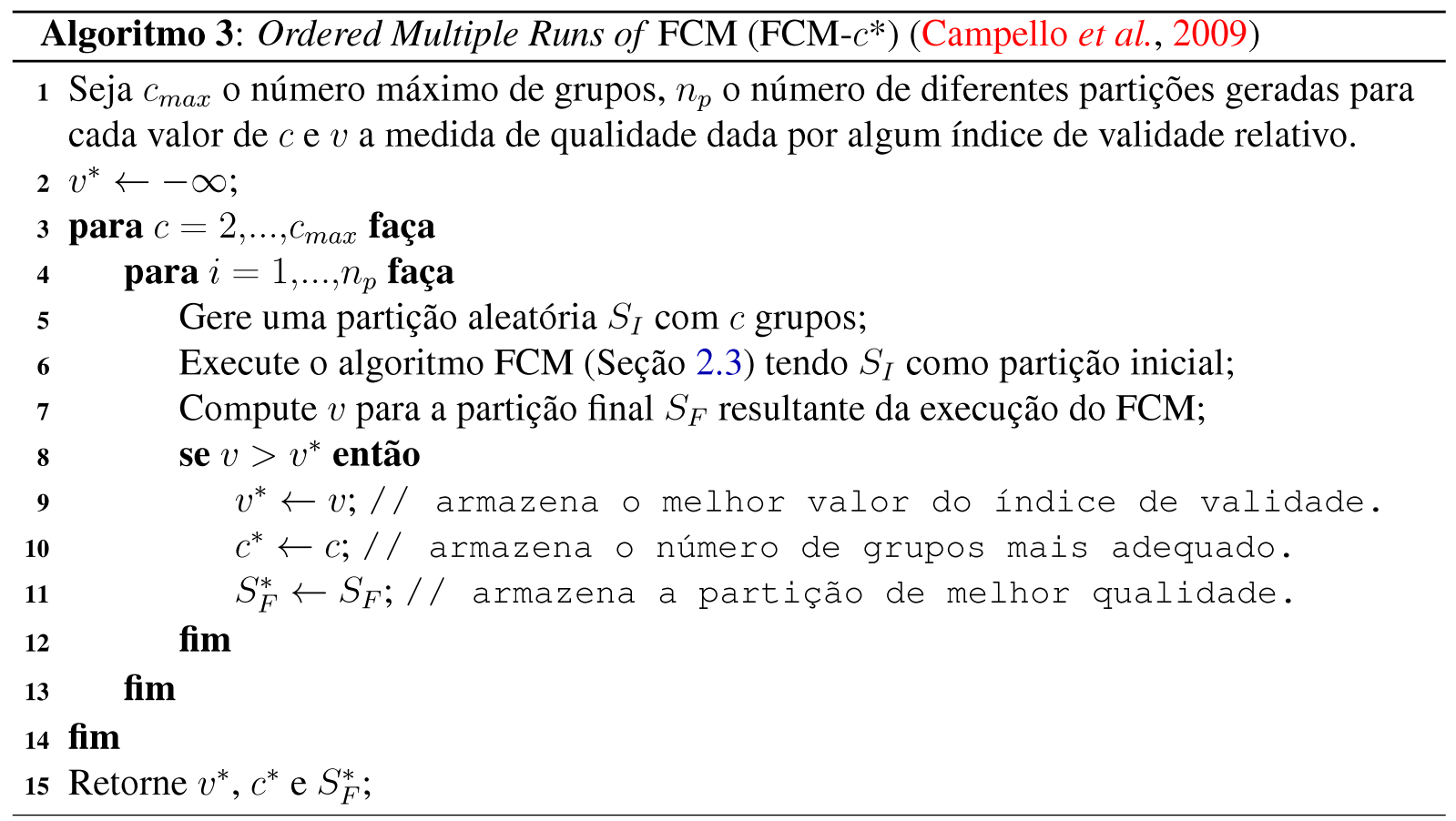

De acordo com o Algoritmo 3, o FCM é executado - com escolha aleatória dos protótipos iniciais $^{3}-n_{p}$ vezes para cada número de grupos $c$, o qual varia de $c_{\min }=2$ até $c_{\max }$. No final desse procedimento existirão $n_{p} \times\left(c_{\max }-c_{\min }+1\right)$ partições, as quais devem ser avaliadas em termos relativos de acordo com um determinado índice de validade relativo. Dado que a complexidade do algoritmo FCM pode ser estimada em $O\left(c^{2} \cdot N \cdot d \cdot n_{t}\right)$ - no qual $c$ é o número de grupos, $N$ é o número de objetos, $d$ é o número de atributos e $n_{t}$ é o número de iterações - o custo computacional do Algoritmo 3 pode ser estimado em $O\left(c_{\max }{ }^{3} \cdot N \cdot d \cdot n_{t} \cdot n_{p}\right)$ (Campello et al., 2009). É importante enfatizar que esse algoritmo é, em princípio, apropriado para índices de maximização, mas pode ser facilmente adaptado para índices de minimização alterando-se os passos 2 e 8 .

\footnotetext{
${ }^{3}$ Convém destacar que outros métodos de inicialização mais sofisticados e eficazes podem ser usados.
} 


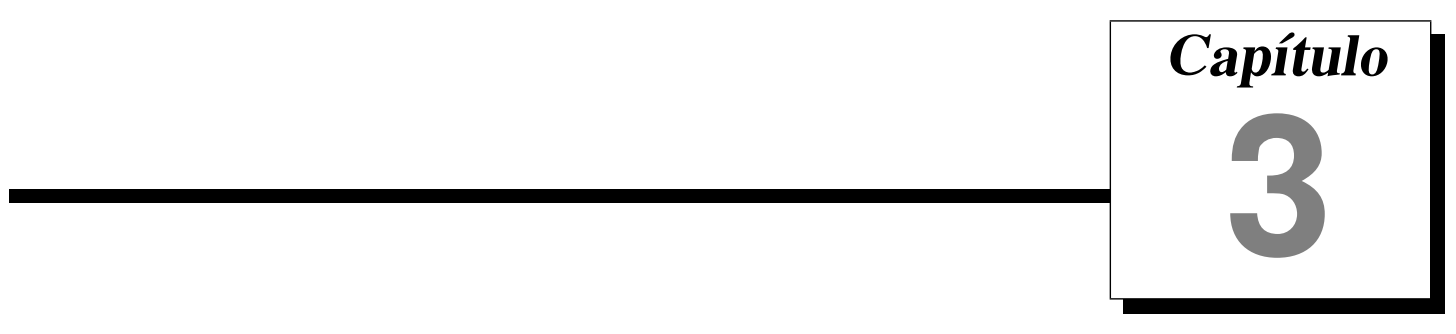

\section{Descoberta de Conhecimento Distribuído}

Atualmente, ambientes baseados em computação distribuída como a internet, intranets e processamento paralelo estão modificando vários aspectos da computação (Park \& Kargupta, 2002; Park et al., 2002). De acordo com (Datta et al., 2006; Tsoumakas, 2004), bases de dados fisicamente distribuídas estão sendo cada vez mais utilizadas para a descoberta de conhecimento. Nesse sentido, a globalização, o business-to-commerce e a colaboração on-line entre as organizações têm aumentado a necessidade de mineração de dados interorganizacionais (e.g., dados que estão fisicamente distribuídos). Como consequência, o problema clássico de análise de dados tem evoluído para um novo domínio multidisciplinar, que além de combinar estatística e aprendizado de máquina, envolve também a computação paralela e/ou distribuída (Kargupta \& Chan, 2000). Essa tendência ocasionou o surgimento de um novo campo de estudo, como uma extensão de KDD (Fayyad et al., 1996a; Piatetsky-Shapiro \& Frawley, 1991), denominado de descoberta de conhecimento distribuído, ou Distributed Knowledge Discovery (DKD) (Zhang et al., 2004; Kargupta et al., 2001; Johnson \& Kargupta, 2000). DKD abrange técnicas e métodos para minerar dados que estão fisicamente distribuídos, lidando com as adversidades impostas por este domínio (Kargupta et al., 2001). Diversos trabalhos encontrados na literatura envolvem DKD (Kargupta et al., 2001; Johnson \& Kargupta, 2000) e, em específico, a mineração de dados distribuídos, ou Distributed Data Mining (DDM) (Da Silva \& Klusch, 2007; Da Silva et al., 2005; Merugu \& Ghosh, 2005; Klusch et al., 2003; Park \& Kargupta, 2002). Assumindo-se que os dados que estão distribuídos em diferentes subconjuntos (sítios de dados) não podem ser centralizados (reunidos em um único local), os métodos e algoritmos projetados para DKD (ou DDM) devem minerá-los considerando-se certas restrições. Tais restrições limitam a transmissão de determinadas informações e/ou grandes quantidades de dados entre os sítios - mesmo com os notáveis avanços no que diz respeito às tecnologias de comunicação dos dados (tais como redes wireless, fibras óticas e dispositivos móveis). Por exemplo, muito frequentemente as grandes redes possuem restrições de segurança e privaci- 
dade (Da Silva \& Klusch, 2007; Merugu \& Ghosh, 2005; Silva \& Klusch, 2006). Além disso, severas limitações de largura de banda podem impossibilitar a interação entre os diferentes sítios de dados (Pedrycz, 2007). Fundamentalmente, os métodos e algoritmos para a descoberta de conhecimento distribuído devem empregar alguma estratégia para lidar com essas possíveis restrições/limitações. Uma estratégia comum e interessante é assumir que os dados presentes nos diferentes sítios podem ser tratados de maneira separada (individual). Nesse sentido, primeiramente realiza-se a análise de dados localmente (em cada sítios de dados, de maneira individual). Em seguida, os resultados parciais (modelos locais) obtidos em cada um dos sítios são enviados para um local comum para que possam ser agregados a fim de construir um resultado global (modelo global) (Klusch et al., 2003). Essa estratégia evita a comunicação direta de dados entre os sítios não violando, assim, as restrições de segurança e privacidade, além de evitar a transmissão de grandes massas de dados. Além disso, métodos e algoritmos para DKD podem lidar com diferentes possibilidades de distribuição dos dados (Kargupta et al., 2001). Em particular, duas formas de distribuição entre os sítios são comumente aceitas: a homogênea (ou horizontalmente particionada) e a heterogênea (ou verticalmente particionada). Na homogênea, os sítios de dados são constituídos de diferentes objetos relativos a um mesmo espaço de atributos. Na heterogênea, os sítios de dados possuem os mesmos objetos, porém em um espaço de atributos diferente. Nesse último caso, cada objeto em cada sítio de dados possui um campo identificador único (para facilitar o relacionamento). Tais formas de distribuição adotam um ponto de vista conceitual em que cada tabela de dados é uma partição de uma única tabela global. Na forma homogênea, a tabela global é horizontalmente particionada. Já na forma heterogênea, a tabela global é verticalmente particionada . Ressalta-se que esse conceito, sugerido em (Da Silva et al., 2005), é meramente ilustrativo, i.e., não é correto afirmar, na realidade, que os dados foram divididos entre os sítios a partir de uma tabela global. As Figuras $3.1 \mathrm{e}$ 3.2 exemplificam, respectivamente, a distribuição homogênea e heterogênea dos dados. Este trabalho aborda a forma de distribuição ilustrada na Figura 3.1 (i.e., a forma horizontal).

\begin{tabular}{|c|c|c|c|c|}
\hline Conta & Cliente & Localização & Último Saque & Saldo \\
\hline 12643324 & 15700 & Belo Horizonte & 1000,00 & 2040,54 \\
\hline 65821943 & 18340 & Curitiba & 568,00 & 6543,12 \\
\hline 31278390 & 02531 & Maceió & 120,00 & $-137,50$ \\
\hline 85532106 & 25896 & São Paulo & 1450,00 & 4210,00 \\
\hline
\end{tabular}

(a)

\begin{tabular}{|c|c|c|c|c|}
\hline Conta & Cliente & Localização & Último Saque & Saldo \\
\hline 84378530 & 30234 & Santos & 600,00 & 1021,30 \\
\hline 18023506 & 00564 & Campinas & 55,00 & $-320,80$ \\
\hline 12705030 & 27944 & São Paulo & 1340,00 & 5400,20 \\
\hline 95730254 & 49754 & Blumenau & 976,00 & 9453,10 \\
\hline
\end{tabular}

(b)

Figura 3.1: Exemplo de distribuição homogênea dos dados. O sítio (a) e o sítio (b) contém os registros de clientes de agências bancárias localizadas em diferentes cidades. 


\begin{tabular}{|c|c|c|c|c|}
\hline Cidade & Temp. $\left({ }^{\circ} \mathbf{C}\right)$ & Umidade (\%) & Nebulosidade & Vento \\
\hline Belo Horizonte & 25 & 45 & Baixa & Fraco \\
\hline Curitiba & 18 & 60 & Alta & Moderado \\
\hline Santos & 27 & 78 & Média & Moderado \\
\hline Maceió & 32 & 37 & Baixa & Forte \\
\hline
\end{tabular}

(a)

\begin{tabular}{|c|c|c|c|c|}
\hline Cidade & Estado & População & Zona Rural & Comércio \\
\hline Belo Horizonte & MG & 2450000 & Baixa & Alto \\
\hline Curitiba & PR & 1820000 & Média & Médio \\
\hline Santos & SP & 418000 & Baixa & Médio \\
\hline Maceió & AL & 936000 & Média & Alto \\
\hline
\end{tabular}

(b)

Figura 3.2: Exemplo de distribuição heterogênea dos dados. O sítio (a) e o sítio (b) contém, respectivamente, dados relativos às condições meteorológicas e à demografia das mesmas cidades. Neste caso o campo "Cidade" é um identificador que relaciona (a) com (b) e vice-versa.

\subsection{Agrupamento de Dados Distribuídos}

Como parte do processo de DKD, o termo agrupamento de dados distribuídos, ou Distributed Data Clustering (DDC) (Silva \& Klusch, 2006; Merugu \& Ghosh, 2005; Klusch et al., 2003) leva em conta que os dados a serem agrupados estão distribuídos em diferentes sítios. Uma solução simplista para este problema é centralizar todos os dados em um único local, de forma que o agrupamento nesse local possa ser computado e transmitido de volta aos locais de origem dos dados (Silva \& Klusch, 2006). No entanto, conforme contextualizado anteriormente, restrições de segurança, privacidade e limitações de largura de banda, impedem a resolução deste problema dessa forma. Nesse sentido, a estratégia comumente adotada (baseada em DKD) consiste em produzir modelos locais por meio da aplicação de algoritmos de agrupamento em cada sítio de dados. A seguir, esses resultados parciais podem ser agregados a fim de construir um resultado global, ou modelo global dos dados (Pedrycz \& Rai, 2008). Existem muitos trabalhos na literatura que tratam desse assunto. Em geral, tais trabalhos abordam algoritmos para a agregação de agrupamentos, ou cluster ensembles (Tumer \& Agogino, 2008; Topchy et al., 2004; Strehl \& Ghosh, 2002; Johnson \& Kargupta, 2000). Além disso, alguns outros algoritmos, como aqueles concebidos para agrupar dados de maneira paralela (visando a eficiência computacional), também podem ser usados para mineração de dados distribuídos (Datta et al., 2009; Modenesi et al., 2007; Tian et al., 2005; Rahimi et al., 2004; Boutsinas \& Gnardellis, 2002; Kwok et al., 2002; Dhillon \& Modha, 2000). Os principais conceitos sobre esses algoritmos serão abordados nas próximas seções. 


\subsubsection{Agregação de Agrupamentos}

A agregação de agrupamentos (cluster ensembles) consiste em agregar diversas partições com o objetivo de obter uma única partição (partição de consenso) que apresente melhor qualidade em relação às partições iniciais (Ghaemi et al., 2009; Hore et al., 2009; Nguyen \& Caruana, 2007; Fred \& Jain, 2005; Topchy et al., 2005). A partir da agregação, espera-se obter resultados de agrupamento melhores sob um ou mais aspectos, tais como: robustez; novidade; reuso; consistência; desempenho e computação distribuída (Ghaemi et al., 2009; Topchy et al., 2005; Strehl \& Ghosh, 2002). De maneira mais pragmática, métodos e algoritmos para a agregação de agrupamentos utilizam uma função de consenso, a qual recebe um conjunto de partições iniciais como entrada e as agrega produzindo uma única partição como resultado final (partição de consenso) (Strehl \& Ghosh, 2002). Diversos trabalhos na literatura abordam métodos e algoritmos para a agregação de agrupamentos, com diferentes maneiras de gerar as partições iniciais e diferentes estratégias de agregação. Como exemplo, podem-se citar trabalhos baseados em: co-associação (Fred \& Jain, 2005); votação (Frossyniotis et al., 2005; Dimitriadou et al., 2001); grafo/hipergrafo (Topchy et al., 2005; Strehl \& Ghosh, 2002); e estatística (Topchy et al., 2004). Em geral, algoritmos para a agregação de agrupamentos são comumente úteis quando existem diversas partições geradas a partir de tarefas como (Pedrycz \& Rai, 2008):

1. A realização de várias execuções de um mesmo algoritmo de agrupamento (com diferentes parâmetros) em um mesmo conjunto de dados;

2. A aplicação de diferentes algoritmos de agrupamento em um mesmo conjunto de dados;

3. A execução de um determinado algoritmo de agrupamento em diferentes sítios de dados;

Essas três tarefas resultam em diversas partições iniciais que podem ser agregadas por meio de uma função de consenso. As tarefas 1 e 2 fazem sentido quando se busca melhorar a qualidade do agrupamento, em geral, com relação ao aspecto robustez (Fred \& Jain, 2005; Topchy et al., 2004). Mais especificamente, a tarefa 2 pode ter como finalidade reunir pontos fortes de diferentes algoritmos de agrupamento almejando melhorias, tal como a descoberta de alguma novidade (i.e., um resultado inédito que não seria encontrado realizando-se o agrupamento de maneira individual) (Strehl \& Ghosh, 2002; Zeng et al., 2002). A tarefa 3, por sua vez, indica que a agregação de agrupamentos pode ser útil na computação distribuída, i.e., quando se deseja agrupar dados que estão distribuídos em diferentes sítios de dados (Tumer \& Agogino, 2008; Nguyen \& Caruana, 2007; Strehl \& Ghosh, 2002). De maneira mais específica, algoritmos para a agregação de agrupamentos podem ser usados na mineração de dados distribuídos, pois, usualmente, constroem um modelo global por meio do compartilhamento de pequenas quantidades de informação em um nível mais alto (abstrato) dos dados, tais como rótulos de grupos, ou qualquer outro tipo de sumarização, as quais podem ser agregadas por meio de uma função de consenso. Isso evita a transmissão de informações confidenciais e/ou grandes quantidades de dados, o que é desejável em agrupamento de dados distribuídos. 


\subsubsection{Agrupamento Paralelo}

A tarefa de mineração de dados usualmente lida com grandes massas de dados, o que torna requisitos como eficiência e escalabilidade imprescindíveis para o sucesso desta prática. Nesse sentido, uma alternativa interessante é a paralelização deste processo. Objetivando-se agrupar dados em tempo hábil, diversos algoritmos que paralelizam o processo de agrupamento têm sido propostos (Datta et al., 2009; Modenesi et al., 2007; Tian et al., 2005; Boutsinas \& Gnardellis, 2002; Kwok et al., 2002; Dhillon \& Modha, 2000). Em particular, um algoritmo de interesse para esse trabalho é o Parallel Fuzzy C-Means (PFCM) (Rahimi et al., 2004). Esse algoritmo é uma extensão do algoritmo FCM (Seção 2.3) capaz de processar os dados de forma paralela. Em outras palavras, considerando a mineração de dados distribuídos, esse algoritmo é capaz de encontrar, para um número fixo de grupos (c), uma partição fuzzy que é precisamente a mesma que encontraríamos se todos os dados (originalmente distribuídos em diferentes sítios) estivessem centralizados em um único local. Devido a essa característica e a forma como compartilha as informações entre os sítios, o algoritmo PFCM tem se mostrado potencialmente útil para agrupamento de dados distribuídos e, especial, para atuar de forma colaborativa, como será visto no Capítulo 4.

\subsubsection{Considerações Finais}

Conforme contextualizado, métodos e algoritmos que realizam a agregação de agrupamentos podem ser úteis para o agrupamento de dados distribuídos. Esses algoritmos são capazes de construir um modelo global dos dados por meio da agregação de diversos agrupamentos (modelos locais) obtidos em diferentes sítios de dados (Tumer \& Agogino, 2008; Nguyen \& Caruana, 2007; Strehl \& Ghosh, 2002). No entanto, nesses domínios (nos quais os dados estão distribuídos), o processo de agregação de agrupamentos pode assumir uma certa complexidade devido a necessidade de obtenção das partições iniciais (em cada um dos sítios de dados) e o uso de uma função de consenso apropriada para agregá-las. Durante esse processo devem ser realizadas transmissões de dados que garantam o envio de informações (modelos locais) a um local comum (para uso da função de consenso) e, em seguida, o envio do modelo global (partição de consenso) a todos os locais envolvidos. Todo esse processo deve ser realizado todas as vezes em que se desejar construir um modelo global dos dados. Nesse sentido, de acordo com (Pedrycz \& Rai, 2008), esses algoritmos assumem uma postura passiva e uma alternativa interessante seria a utilização de algoritmos para agrupamento colaborativo. Agrupamento colaborativo, inicialmente idealizado em (Pedrycz, 2002), têm se mostrado capaz de lidar com dados distribuídos por meio de um processo dinâmico que evita a transmissão de informações confidenciais e/ou grandes quantidades de dados. Em particular, algumas variantes do algoritmo FCM (Seção 2.3) têm se mostrado capazes de atuarem de maneira colaborativa. Como exemplo, pode-se citar o algoritmo PFCM (Rahimi et al., 2004) que devido a natureza distribuída/paralela de seu processo, pode realizar agrupamento colaborativo especialmente em cenários nos quais se assume que os dados distribuídos são de uma mesma população. Tais suposições, bem como os 
principais conceitos de agrupamento de dados colaborativo, serão abordados de maneira mais detalhada no próximo capítulo, no qual também será descrito o algoritmo Collaborative Fuzzy Clustering (CFC) (Pedrycz \& Rai, 2008), que é uma extensão do algoritmo FCM para realizar agrupamento colaborativo. 


\section{Capítulo}

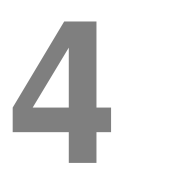

\section{Algoritmos para Agrupamento Fuzzy Colaborativo}

O processo de agrupamento colaborativo requer que os algoritmos de agrupamento, aplicados em diferentes sítios de dados, comuniquem ativamente suas descobertas entre si (Pedrycz \& Rai, 2008). Tais descobertas consistem de informações (resultados) de agrupamentos em um nível mais alto (abstrato) dos dados denominado de grânulos de informação (Pedrycz, 2002). Esse conceito pode evitar a comunicação de informações confidenciais, bem como a transmissão de grandes quantidades de dados entre os sítios. Por isso, agrupamento colaborativo tem se mostrado particularmente útil para o campo que se convencionou denominar de Distributed Knowledge Discovery (DKD) (Kargupta et al., 2001; Johnson \& Kargupta, 2000) e, em específico, para Distributed Data Clustering (DDC) (Silva \& Klusch, 2006; Merugu \& Ghosh, 2005; Klusch et al., 2003). A idéia de agrupamento colaborativo foi introduzida em (Pedrycz, 2002) e tem sido abordada em diversos trabalhos nos últimos anos (Forestier et al., 2010; Yu et al., 2009; Pedrycz \& Rai, 2008; Falcón et al., 2008; Forestier et al., 2008; Falcon et al., 2007; Pedrycz, 2007; Yu et al., 2007; Mitra et al., 2006). Métodos e algoritmos para agrupamento colaborativo têm como objetivo revelar estruturas de grupos que são (aproximadamente) comuns em diferentes sítios de dados (Pedrycz, 2002). Essa definição implicitamente sugere que os dados (amostra) em cada um dos sítios foram gerados a partir de diferentes populações. Nesse caso, não se espera a identificação de grupos de populações muito diferentes em cada um dos sítios de dados. Contrariamente, espera-se que cada sítio de dados seja beneficiado apenas por informações (relativamente) similares comunicadas por outros sítios. Nesse sentido, o processo de agrupamento colaborativo permite que um algoritmo de agrupamento aplicado em um determinado local (sítio de dados) tenha seu resultado final influenciado por informações transmitidas de outros locais. Cenários nos quais algoritmos para agrupamento 
colaborativo podem ser potencialmente empregados incluem (Pedrycz, 2002):

- Suponha que em uma determinada instituição financeira deseja-se agrupar os dados de seus clientes. Contudo, por questões de confidencialidade, não é permitido o compartilhamento desses dados entre as diferentes agências bancárias desta instituição. Além disso, os dados dessas agências não podem ser centralizados por questões de desempenho computacional. Assim, deseja-se aplicar algoritmos para agrupar dados de clientes em cada agência bancária (individualmente) e, ao mesmo tempo, compartilhar descobertas individuais (colaborar) sem comprometer restrições de privacidade e de desempenho computacional.

- Em uma grande rede de lojas não é desejável reagrupar completamente os dados de seus clientes sempre que seus dados forem atualizados, i.e., não se deseja simplesmente aplicar o algoritmo de agrupamento nos dados atualizados, desprezando-se os grupos já induzidos anteriormente. Neste caso, seria mais conveniente, por exemplo, executar algoritmos de agrupamento incrementalmente, refinando-se grupos obtidos em um tempo $t$ a partir de objetos obtidos em um tempo $(t+1)$. Nesse sentido, haveria uma colaboração entre as partições de dados induzidas em diferentes instantes de tempo.

Seguindo a linha desses exemplos, algoritmos para agrupamento colaborativo podem ser, então, úteis quando não é viável o compartilhamento dos dados (brutos) que estão distribuídos, entretanto, busca-se extrair conhecimento (em comum) presente nesses dados. Dessa maneira, espera-se que, na prática, essas ferramentas sejam importantes para organizações situadas em diferentes localizações, mas com interesse em comum de realizar descobertas nos dados que estão distribuídos. Como exemplo, podem-se citar grandes financeiras, centros comerciais, redes de hospitais, instituições públicas, etc. Em particular, organizações concorrentes poderiam usufruir desse mecanismo para obter maior conhecimento sobre seus dados sem a necessidade de revelar informações sigilosas entre si. Como exemplo, lojas concorrentes, de uma determinada localidade, poderiam agrupar seus dados de forma colaborativa buscando identificar o perfil de seus clientes nessa região.

\subsection{Formalização do Problema}

O problema de agrupar dados de maneira colaborativa pode ser definido da seguinte maneira: assumindo um número finito de subconjuntos de dados (sítios de dados), deseja-se desenvolver estruturas de grupos em cada um desses sítios levando-se em conta um certo nível de interação entre esses diferentes locais. Essa interação pode ser realizada por meio da comunicação de descobertas na forma de grânulos de informação (Pedrycz \& Rai, 2008). De maneira mais formal, considere $P$ sítios de dados, $D[1], D[2], \ldots, D[P]$, cada um desses consistindo de $N[1], N[2], \ldots, N[P]$ objetos definidos em um mesmo espaço $d$-dimensional de atributos - i.e., assumindo-se a distribuição homogênea dos dados (abordado na Seção 3). A finalidade do agrupamento colaborativo é identificar estruturas (agrupamentos) nos dados pela obtenção de 
$c[1], c[2], \ldots, c[P]$ grupos nos respectivos sítios de dados (uma partição de dados para cada sítio), de maneira que a formação de cada uma dessas estruturas seja influenciada (em um certo nível) por outras estruturas em formação em outros locais.

\subsection{Algoritmo CFC — Nível de Interação Fixo}

Nesta seção é apresentada uma extensão do algoritmo FCM (Seção 2.3) para agrupamento colaborativo, a qual é aqui chamada de Collaborative Fuzzy Clustering (CFC) (Pedrycz \& Rai, 2008). A primeira fase do algoritmo CFC tem como objetivo identificar agrupamentos nos dados de cada um dos $P$ sítios, $D[1], D[2], \ldots, D[P]$, por meio da obtenção de $c[1], c[2], \ldots, c[P]$ grupos em suas respectivas partições. Como esse algoritmo assume que o número de grupos é conhecido a priori, e que este é invariante nos diferentes sítios de dados, tem-se, então, $c[1]=$ $c[2]=\ldots=c[P]=c$ ( $c$ deve ser informado pelo usuário). Portanto, nessa primeira fase, buscase revelar (sem nenhum tipo de interação entre os sítios) partições com $c$ grupos em cada um dos $P$ sítios de dados. O algoritmo FCM (Seção 2.3) é usado nessa fase, que tem como resultado um conjunto de protótipos e de matrizes de partição. Em princípio, as descobertas em cada sítio de dados poderiam então ser comunicadas por meio dos protótipos ou das matrizes de partição. Entretanto, os sítios de dados frequentemente possuem diferentes números de objetos, tornando o compartilhamento de descobertas a partir das matrizes de partição mais complicado. Neste contexto, o uso dos protótipos como veículos para a transmissão das descobertas parece ser mais apropriado. Resumidamente, os protótipos podem representar os grupos obtidos, sumarizando a informação codificada em diferentes números de objetos.

Considere então que os resultados produzidos em um determinado sítio de dados podem ser claramente identificados pelo índice do respectivo sítio. Assim, para o $i i$-ésimo sítio, sua matriz de partição (que armazena as pertinências dos objetos aos grupos) é denotada por $\mathrm{U}[i i]=\left[u_{i j}[i i]\right](i=1, \ldots, c ; j=1,2, \ldots, N[i i] ; i i=1, \ldots, P)$, enquanto que os protótipos correspondentes aos grupos podem ser representados por $\mathbf{v}_{1}[i i], \mathbf{v}_{2}[i i], \ldots, \mathbf{v}_{c}[i i]$. Por simplicidade e sem perda de generalidade, assuma que o agrupamento colaborativo acontecerá em um único sítio de dados, e.g., em $D[i i]$. Neste caso, todos os outros sítios $D[1], D[2], \ldots, D[i i-1], D[i i+1], \ldots, D[P]$ comunicam seus respectivos protótipos $\mathbf{v}_{i}[j j]$ $(i=1,2, \ldots, c ; j j=1,2, \ldots, i i-1, i i+1, \ldots, P)$ para $D[i i]$. A partir desses protótipos podese gerar uma matriz de partição induzida, $\widetilde{\mathbf{U}}[i i \mid j j] \in \Re^{c \times N[i i]}$, na qual os índices $i i$ e $j j$ se referem aos sítios de dados que participam dessa interação. Ou seja, por meio dos protótipos $\mathbf{v}_{1}[j j], \mathbf{v}_{2}[j j], \ldots, \mathbf{v}_{c}[j j]$, correspondentes ao $j j$-ésimo sítio de dados, pode-se induzir uma matriz de partição $\widetilde{\mathbf{U}}[i i \mid j j]$ sobre o sítio de dados $D[i i]$. A matriz de partição induzida pode ser calculada por meio da Eq. (2.5) do algoritmo FCM (Algoritmo 2). Dessa forma, a pertinência do $j$-ésimo objeto ao $i$-ésimo grupo pode ser calculada pela Eq. (4.1):

$$
\widetilde{u}_{i j}[i i \mid j j]=\frac{1}{\sum_{p=1}^{c}\left(\frac{\left\|\mathbf{x}_{j}[i i]-\mathbf{v}_{i}[j j]\right\|}{\left\|\mathbf{x}_{j}[i i]-\mathbf{v}_{p}[j j]\right\|}\right)^{2}}
$$


Na Eq. (4.1), $\mathbf{x}_{j} \in D[i i](j=1,2, \ldots, N[i i])$ e $\mathbf{v}_{i} \in D[j j](i=1,2, \ldots, c)$. Calculando-se as pertinências dos objetos aos grupos para todos os sítios de dados, $D[1], \ldots, D[i i-1], D[i i+$ $1], \ldots, D[P]$, obtêm-se $P-1$ matrizes de partição induzidas, i.e., $\widetilde{\mathbf{U}}[i i \mid 1], \widetilde{\mathbf{U}}[i i \mid 2], \ldots, \widetilde{\mathbf{U}}[i i \mid i i-1]$, $\widetilde{\mathbf{U}}[i i \mid i i+1], \ldots, \tilde{\mathbf{U}}[i i \mid P]$.

O agrupamento fuzzy colaborativo realizado pelo algoritmo CFC é guiado pela minimização da função objetivo formulada em (4.2):

$$
Q[i i]=\sum_{j=1}^{N[i i]} \sum_{i=1}^{c} u_{i j}^{2}[i i] d_{i j}^{2}+\beta \sum_{\substack{j j=1 \\ j \neq i i}}^{P} \sum_{j=1}^{N[i i]} \sum_{i=1}^{c}\left(u_{i j}[i i]-\widetilde{u}_{i j}[i i \mid j j]\right)^{2} d_{i j}^{2}
$$

na qual $\beta$ é um valor não negativo e $d_{i j}^{2}=\left\|\mathbf{x}_{j}-\mathbf{v}_{i}[i i]\right\|^{2}$. Essa função objetivo consiste de dois termos. O primeiro termo é a soma das distâncias ponderadas pelas pertinências entre os objetos e protótipos de $D[i i]$ — ou seja, esse termo é equivalente a função objetivo $J$ em (2.3) do algoritmo FCM (Seção 2.3). O segundo termo reflete o impacto proveniente das estruturas formadas em todos os outros sítios de dados. Nesse sentido, esse termo visa capturar a minimização das distâncias entre as matrizes de partição a serem otimizadas e as respectivas matrizes induzidas. É importante enfatizar que nesse segundo termo foi observado um possível problema de correspondência entre os protótipos locais e os protótipos transmitidos por outros sítios de dados. Particularmente, pode-se notar que não há garantias de que as linhas da matriz de partição a ser otimizada e as respectivas linhas das matrizes induzidas correspondem aos mesmos grupos (ou pelo menos aos grupos mais similares). Esse fato pode dificultar o processo de minimização. Por fim, o valor do coeficiente $\beta$ define o nível de interação entre os sítios de dados. Em outras palavras, o valor de $\beta$ determina o nível de influência que as estruturas descobertas nos outros sítios de dados têm na formação da estrutura local ( $D[i i]$ neste caso). Valores altos de $\beta$ implicam em uma forte colaboração, enquanto que para $\beta=0$ não existe colaboração — neste caso o problema se resume em obter $P$ agrupamentos independentes, limitados aos correspondentes sítios de dados. Levando-se em conta que o usuário informa o número de grupos, $c$, e um valor não negativo para o nível de interação, $\beta$, o algoritmo CFC pode ser sumarizado em duas fases (Pedrycz \& Rai, 2008):

\section{Fase Inicial:}

Execute o algoritmo FCM (Seção 2.3) para cada sítio de dados, produzindo, assim, uma coleção de protótipos $\mathbf{v}_{i}[i i](i=1,2, \ldots, c ; i i=1, \ldots, P)$.

\section{Fase Colaborativa:}

Nesta fase, que se constitui de vários estágios de colaboração, ocorrem as comunicações de protótipos (grânulos de informação) entre os sítios de dados. Para cada um dos estágios de colaboração busca-se minimizar a Eq. (4.2) para cada sítio de dados, $D[i i](i i=1, \ldots, P)$. Esta minimização pode ser realizada por meio de um processo iterativo, que compreende o cálculo da matriz de partição local — Eq. (4.3) - e o cálculo dos protótipos — Eq. (4.4): 


$$
\begin{gathered}
u_{i j}[i i]=\frac{1}{\sum_{p=1}^{c}\left(d_{i j}^{2}\right) /\left(d_{p j}^{2}\right)}\left[1-\sum_{p=1}^{c} \frac{\beta \sum_{\substack{j j=1 \\
j j \neq i i}}^{P} \widetilde{u}_{p j}[i i \mid j j]}{[1+\beta(P-1)]}\right]+\frac{\beta \sum_{\substack{j j=1 \\
j \neq i i}}^{P} \widetilde{u}_{i j}[i i \mid j j]}{[1+\beta(P-1)]} \\
v_{i h}[i i]=\frac{\sum_{j=1}^{N[i i]} u_{i j}^{2}[i i] x_{j h}+\beta \sum_{\substack{j j=1 \\
j j \neq i i}}^{P} \sum_{j=1}^{N[i i]}\left(u_{i j}[i i]-\widetilde{u}_{i j}[i i \mid j j]\right)^{2} x_{j h}}{\sum_{j=1}^{N[i i]} u_{i j}^{2}[i i]+\beta \sum_{\substack{j j=1 \\
j \neq i i}}^{P} \sum_{j=1}^{N[i i]}\left(u_{i j}[i i]-\widetilde{u}_{i j}[i i \mid j j]\right)^{2}}
\end{gathered}
$$

Nestas equações, $i=1,2, \ldots, c ; j=1,2, \ldots, N[i i] ; h=1,2, \ldots, d$ e $d_{i j}^{2}=\left\|\mathbf{x}_{j}-\mathbf{v}_{i}[i i]\right\|^{2}$. A Fase Colaborativa do algoritmo CFC pode ser sumarizada pelo Algoritmo 4.

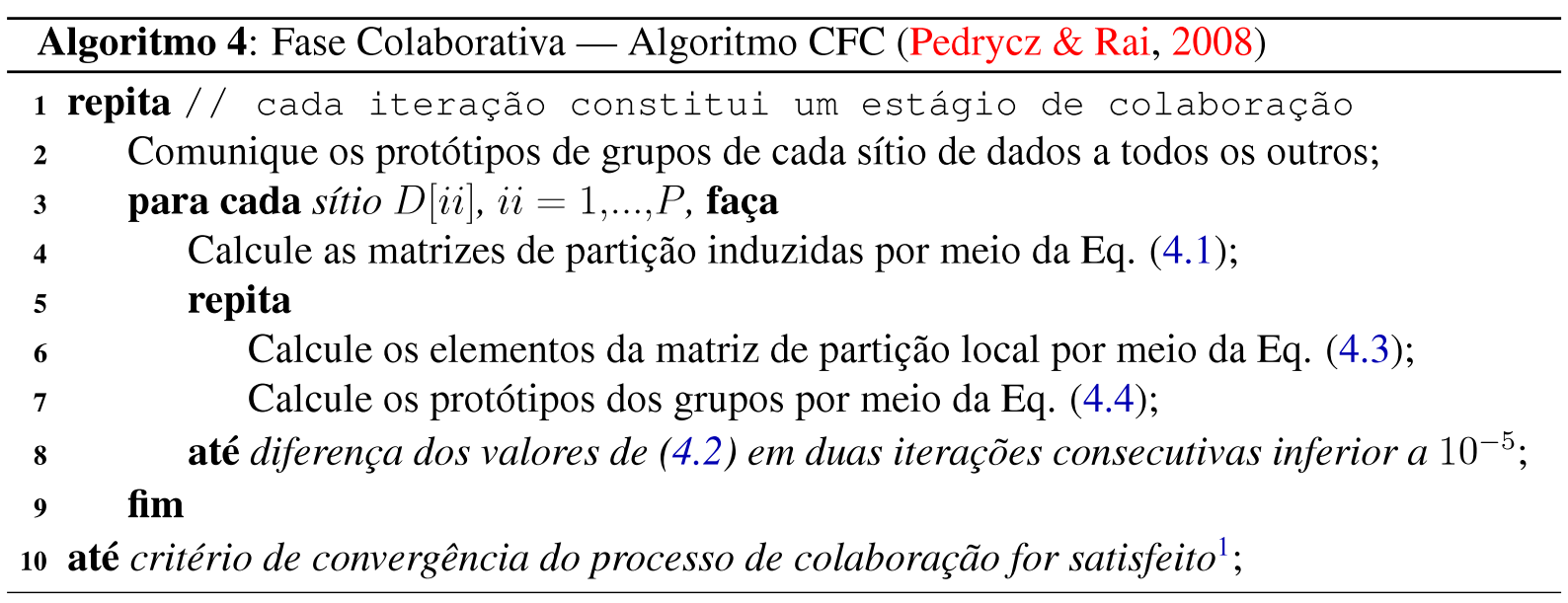

Em (Pedrycz \& Rai, 2008) é também proposta uma forma de avaliar a qualidade do processo de colaboração realizado pelo algoritmo CFC. Especificamente, o índice de qualidade proposto $(W)$ pode ser usado para monitorar a evolução do processo de colaboração durante os estágios de colaboração. Considere uma matriz de partição $\mathbf{U}[i i]=\left[u_{i j}\right], i=1,2, \ldots, c, j=1,2, \ldots N[i i]$, obtida no sítio de dados $D[i i]$. A partir dessa matriz de partição pode-se induzir uma matriz de proximidade, Prox $=[\operatorname{prox}(r, s)](r, s=1,2, \ldots, N[i i])$ dada pela Eq. (4.5):

$$
\operatorname{prox}(r, s)=\sum_{i=1}^{c} \min \left(u_{i r}, u_{i s}\right)
$$

Assumindo a matriz de partição a ser otimizada, $\mathbf{U}[i i]$, e todas as outras matrizes de partição induzidas, $\widetilde{\mathbf{U}}[i i \mid 1], \widetilde{\mathbf{U}}[i i \mid 2], \ldots, \widetilde{\mathbf{U}}[i i \mid i i-1], \widetilde{\mathbf{U}}[i i \mid i i+1], \ldots, \widetilde{\mathbf{U}}[i i \mid P]$, pode-se quantificar a consistência entre a estrutura em formação no sítio de dados $D[i i]$ e as estruturas existentes nos outros sítios por meio da Eq. (4.6):

\footnotetext{
${ }^{1}$ Nesse trabalho o algoritmo é interrompido quando os protótipos não se alterarem significativamente em duas iterações consecutivas.
} 


$$
W[i i]=\frac{1}{\left(N[i i]^{2} / 2\right)} \sum_{\substack{j j=1 \\ j \neq i i}}^{P}\|\operatorname{Prox}(\mathbf{U}[i i])-\operatorname{Prox}(\tilde{\mathbf{U}}[i i \mid j j])\|
$$

$\mathrm{Na}$ Eq. (4.6), a distância entre as matrizes de proximidade é dada pela distância de Hamming, a qual pode ser computada pela Eq. (4.7):

$$
\|\operatorname{Prox}(\mathbf{U}[i i])-\operatorname{Prox}(\widetilde{\mathbf{U}}[i i \mid j j])\|=\sum_{r=1}^{N[i i]} \sum_{s>r}^{N[i i]}|\operatorname{prox}(r, s)[i i]-\operatorname{prox}(r, s)[i i \mid j j]|
$$

A cada estágio de colaboração (veja o Algoritmo 4) é possível calcular o índice (global) $W$, por meio da Eq. (4.8):

$$
W=W[1]+W[2]+\ldots+W[P]
$$

Os proponentes do algoritmo CFC (Pedrycz \& Rai, 2008) consideram que os valores de $W$ obtidos em sucessivos estágios de colaboração podem ser usados como um indicador do progresso e da qualidade do processo de colaboração. Valores baixos desse índice indicam maior consistência entre as $P$ estruturas. Nesse sentido, a minimização de $W$ pode ser usada como um critério de convergência do processo de colaboração realizado pelo algoritmo CFC. Além disso, os autores argumentam que $W$ também pode ser usado para estimar o valor do nível de interação $\beta$. Especificamente, $W$ pode ser tratado como uma função de $\beta$, i.e., $W=W(\beta)$. Neste contexto, um procedimento utilizado consiste em fornecer diferentes valores de $\beta$ ao algoritmo CFC, de maneira que se possa computar, para cada $\beta$, o correspondente valor de $W$ (minimizado após vários estágios de colaboração). O valor ótimo de $\beta$ é aquele que produz o menor valor de $W$. Os experimentos descritos na Seção 5.2 ilustram esse procedimento e também abordam suas limitações. Em resumo, vale antecipar que $W$ apresenta alguns problemas. Por exemplo, uma mesma matriz de proximidade pode ser produzida por matrizes de partição significativamente diferentes. A Figura 4.1 ilustra duas matrizes de partição distintas que, entretanto, fornecem o mesmo valor de proximidade $-\operatorname{prox}(r, s)=0,6$. Neste caso, a Eq. (4.6) sugere que as estruturas de agrupamento representadas pelas matrizes são consistentes, o que não é verdade. Por exemplo, note que, numa interpretação probabilística, o objeto $s$ nitidamente pertence ao grupo $c_{2}$ de acordo com $\mathbf{U}[i i]$, enquanto que $\widetilde{\mathbf{U}}[i i \mid j j]$ não permite identificar claramente o grupo que gerou este objeto. 


\begin{tabular}{|c|c|c|}
\hline & $r$ & $s$ \\
\hline$c_{1}$ & 0,7 & 0,3 \\
\hline$c_{2}$ & 0,3 & 0,7 \\
\hline
\end{tabular}

Matriz de partição $\mathbf{U}[i i]$

\begin{tabular}{|c|c|c|}
\hline & $r$ & $s$ \\
\hline$c_{1}$ & 0,1 & 0,5 \\
\hline$c_{2}$ & 0,9 & 0,5 \\
\hline
\end{tabular}

Matriz de partição $\widetilde{\mathbf{U}}[i i \mid j j]$

Figura 4.1: Matrizes de partição equivalentes para o conceito de matriz de proximidade.

\subsection{Algoritmos Propostos para Agrupamento Colaborativo}

O algoritmo CFC (Pedrycz \& Rai, 2008), apresentado na Seção 4.2, depende de dois parâmetros que devem ser informados pelo usuário: o nível de interação $\beta$ e o número de grupos $c$. Para superar a dependência do primeiro parâmetro $(\beta)$, foram desenvolvidos nesse trabalho dois novos algoritmos, chamados de CFC- $\beta_{d}$ e CFC- $\beta_{f}$. Esses algoritmos são capazes de estimar (a partir dos dados) os níveis de interação entre os pares de sítios. Com respeito ao último parâmetro (c), outros dois algoritmos, chamados de CFCM- $c^{*}$ e CFCM- $\beta_{f}-c^{*}$ e que são capazes de estimar o número de grupos automaticamente a partir dos dados, foram desenvolvidos. Além disso, o algoritmo Parallel Fuzzy C-Means (PFCM) (Rahimi et al., 2004), abordado nas Seções 3.1.2 e 3.1.3, também pode ser útil para agrupamento colaborativo, especialmente em cenários nos quais se assume que os dados distribuídos são de uma mesma população. Contudo, esse algoritmo também necessita da informação do número de grupos $(c)$. Para superar essa limitação, foi desenvolvida uma extensão desse algoritmo, chamada de PFCM- $c^{*}$, a qual é capaz de estimar automaticamente o número de grupos. As Seções 4.3.1, 4.3.2 e 4.3.3 descrevem em detalhes esses novos algoritmos. Conforme esperado, na prática alguns algoritmos podem ser mais interessantes do que outros. Os principais fatores que influeciam na escolha do algoritmo a ser utilizado são: as suposições sobre os dados (i.e., dados que são de uma mesma população ou de diferentes populações) e a dificuldade em se definir os parâmetros dos algoritmos. Deste modo, na Seção 4.4 é apresentada uma árvore de decisão que tem como objetivo auxiliar o usuário interessado em escolher, dentre os algoritmos disponíveis, o mais apropriado para o problema que se tem em mãos. As análises das complexidades de tempo, espaço e comunicação de todos os algoritmos também estão sumarizadas nesta seção.

\subsubsection{Algoritmos CFC- $\beta_{d}$ e CFC- $\beta_{f}$ - Nível de Interação Variável}

O algoritmo CFC (Seção 4.2), requer que o usuário informe o nível de interação $\beta$, o qual é mantido constante (fixo) para todos os pares de sítios durante todos os estágios de colaboração. Com o objetivo de estimar automaticamente (a partir dos dados) os níveis de interação entre pares de sítios dois novos algoritmos foram desenvolvidos. Especificamente, esses novos algoritmos ajustam (estimam) um valor particular $\beta[i i \mid j j], i i, j j=1,2, \ldots, P$, para cada par de 
sítio de dados. Nesse sentido, se as estruturas dos grupos entre dois sítios quaisquer são muito diferentes, então $\beta$ deveria ser baixo, fornecendo uma fraca colaboração entre esses dois sítios. Por outro lado, para pares de sítios muito similares espera-se um valor alto de $\beta$, o que sugere uma forte colaboração entre esses sítios. De maneira mais formal, considere dois sítios de da$\operatorname{dos}(D[i i]$ e $D[j j])$ e seus respectivos protótipos $\left(\mathbf{v}_{i}[i i]\right.$ e $\left.\mathbf{v}_{i}[j j], i=1, \ldots, c\right)$. Suponha que os protótipos $\mathbf{v}_{i}[j j]$ foram enviados para o sítio de dados $D[i i]$. Seguindo a filosofia do algoritmo CFC (Seção 4.2) pode-se calcular, então, a matriz de partição induzida $\widetilde{\mathbf{U}}[i i \mid j j]$ usando a Eq. (4.1). A partir dessa matriz, pode-se computar a função objetivo (induzida), de acordo com a Eq. (4.9):

$$
\widetilde{J}[i i \mid j j]=\sum_{j=1}^{N[i i]} \sum_{i=1}^{c} \widetilde{u}_{i j}^{2}[i i \mid j j]\left\|\mathbf{x}_{j}-\mathbf{v}_{i}[j j]\right\|^{2}
$$

onde $\widetilde{u}_{i j}^{2}[i i \mid j j]$ é um elemento da matriz de partição induzida $\widetilde{\mathbf{U}}[i i \mid j j]$ e $\mathbf{x}_{j} \in D[i i]$. Dado que $J[i i]$ é a função objetivo (2.3) computada a partir dos objetos e protótipos locais do sítio $D[i i]$, o nível de interação $\beta[i i \mid j j]$ entre dois sítios $(D[i i]$ e $D[j j])$ para um dado estágio de colaboração pode ser definido pela Eq. (4.10):

$$
\beta[i i \mid j j]=\min \left\{1, \frac{J[i i]}{\widetilde{J}[i i \mid j j]}\right\}
$$

Nesta equação, o termo $\widetilde{J}[i i \mid j j]$ é tipicamente maior do que o termo $J[i i]$, pois o primeiro é computado com os protótipos enviados pelo $j j$-ésimo sítio de dados, enquanto que o último foi otimizado para o próprio $i$-ésimo sítio. Assim, o nível de interação $\beta[i i \mid j j]$ pode expressar a seguinte idéia: um valor de $\beta[i i \mid j j]$ próximo de 1 sugere que as estruturas dos sítios $D[i i] \mathrm{e}$ $D[j j]$ são bem parecidas e, portanto, devem ter uma forte colaboração entre si, enquanto que um valor de $\beta[i i \mid j j]$ próximo de 0 sugere que as estruturas são muito diferentes e, assim, devem ter uma fraca colaboração entre si.

A idéia capturada pela Eq. (4.10) foi incorporada no algoritmo CFC com o objetivo de dinamicamente ajustar o nível de interação entre todos os possíveis pares de sítios de dados em cada estágio de colaboração. Esse algoritmo modificado é chamado de CFC- $\beta_{d}$. A partir desse algoritmo, pode-se derivar um outro algoritmo computacionalmente mais eficiente. Esse algoritmo, chamado de CFC- $\beta_{f}$, estima os valores de $\beta[i i \mid j j]$ para cada par de sítio de dados somente um vez — antes dos estágios de colaboração — e mantém esses valores fixos durante toda a Fase Colaborativa. É importante ter em mente que ao usar esses algoritmos (bem como o algoritmo original CFC) o usuário implicitamente está assumindo que os dados dos diferentes sítios são provenientes de diferentes populações, pois nesses algoritmos espera-se que somente os sítios de dados que possuem estruturas (relativamente) similares se beneficiem do processo de colaboração. Em outras palavras, assume-se a priori que não há razões para o compartilha- 
mento de informações entre sítios de dados muito diferentes, pois tais informações poderiam comprometer o processo de agrupamento como um todo.

\subsubsection{Algoritmos CFCM- $c^{*}$ e CFCM- $\beta_{f}-c^{*}$ - Número de Grupos Variável}

Os algoritmos apresentados nas Seções 4.2 e 4.3.1 (CFC, CFC- $\beta_{d}$ e CFC- $\left.\beta_{f}\right)$ necessitam da informação do número de grupos $(c)$. Além disso, nesses algoritmos, o valor de $c$ é mantido constante (fixo) em todos os sítios de dados durante todos os estágios de colaboração. Com o objetivo de relaxar essa limitação, foram desenvolvidos dois novos algoritmos que estimam automaticamente (a partir dos dados) o número de grupos para cada um dos sítios. Esses algoritmos são chamados de CFCM- $c^{*}$ e CFCM- $\beta_{f}-c^{*}$.

Considerando a notação adotada na Seção 4.1, assuma $P$ sítios de dados, $D[1], D[2], \ldots, D[P]$, com $N[1], N[2], \ldots, N[P]$ objetos, respectivamente. Busca-se aqui descobrir estruturas com $c[1], c[2], \ldots, c[P]$ grupos nos respectivos sítios. O algoritmo FCM (Seção 2.3) é usado nessa tarefa inicial (de maneira individual, em cada um dos sítios). Dado que os resultados produzidos podem ser claramente identificados pelo índice de seu respectivo sítio, para o ii-ésimo sítio de dados, a matriz de partição computada pelo FCM é denotada por $\mathbf{U}[i i]=\left[u_{i j}[i i]\right], i=1, \ldots, c[i i], j=1, \ldots, N[i i]$ e os protótipos são definidos como $\mathbf{v}_{1}[i i], \mathbf{v}_{2}[i i], \ldots, \mathbf{v}_{c[i i]}[i i]$. Por simplicidade, considere que o agrupamento colaborativo é realizado em um único sítio de dados, e.g., em $D[i i]$. Nesse caso, todos os outros sítios, $D[1], D[2], \ldots, D[i i-1], D[i i+1], \ldots, D[P]$, comunicam seus respectivos protótipos $\mathbf{v}_{i}[j j](i=1,2, \ldots, c[j j] ; j j=1,2, \ldots, i i-1, i i+1, \ldots, P)$ para $D[i i]$. Esses protótipos podem ser vistos como representantes de seus grupos porque sumarizam a informação codificada em diferentes números de objetos. Para tornar esse ponto mais claro e, assim, definir o conceito de representatividade usado nos algoritmos dessa seção, considere a obtenção de uma partição rígida (crisp) a partir de uma matriz de partição fuzzy induzida em um sítio de dados específico, $D[j j]$, pela "classificação" de seus objetos de acordo com suas pertinências aos grupos desse sítio. O $j$-ésimo objeto de $D[j j]$ é classificado ao $i$-ésimo grupo fuzzy de $D[j j]$ se a sua pertinência $u_{i j}[j j]$ for a maior pertinência deste objeto relativamente a qualquer outro grupo, i.e., se $u_{i j}[j j] \geq u_{p j}[j j]$ para todo $p \in\{1, \ldots, c[j j]\}, p \neq i$. Dessa maneira, é possível computar o número de objetos "classificados" em cada grupo fuzzy de $D[j j]$. Considere que este número seja representado por $n_{i}[j j], i=1,2, \ldots, c[j j]$. De maneira aproximada, tal número indiretamente captura o quão representativo é um dado protótipo de $D[j j]$ no que diz respeito ao grupo por este modelado. Contudo, esta abordagem pode ocasionar em uma determinada perda de informação, pois os valores das pertinências do agrupamento fuzzy foram indiretamente discretizados, e pertencem ao conjunto $\{0,1\}$. Em outras palavras, obteve-se uma partição rígida, na qual cada objeto pertence a um único grupo, analogamente ao que se obteria usando o algoritmo $k$-means (Seção 2.2). Para relaxar essa restrição, de maneira simples, cada $n_{i}[j j]$ poderia ser alternativamente computado por meio da medida sigma-count (Pedrycz \& Gomide, 2007). Essa medida utiliza as pertinências dos objetos aos grupos para calcular a representatividade de cada protótipo, fazendo melhor uso da informação proporcionada pelos grupos fuzzy de $D[j j]$. A 
medida sigma-count para o $i$-ésimo grupo fuzzy de $D[j j]$ é dada pela Eq. (4.11):

$$
n_{i}[j j]=\sum_{j=1}^{N[j j]} u_{i j}[j j]
$$

Adicionalmente, é possível incorporar nesta equação um fator de interesse, $\gamma_{i}$, que reflete o quão interessante e/ou confiável são os grupos de uma determinada partição de dados. Por exemplo, pode-se computar $n_{i}[j j]=\gamma_{i} \sum u_{i j}[j j], \gamma_{i} \in[0,1]$. Um alto valor de $\gamma_{i}($ e.g., 0,95$)$ sugeriria que o grupo $i$ é muito interessante para a colaboração, enquanto que um baixo valor sugeriria o contrário. Assim, um especialista de domínio poderia eventualmente tornar alguns grupos mais interessantes para a colaboração do que outros. Como um caso particular, pode-se assumir $\gamma_{1}, \gamma_{2}, \ldots, \gamma_{c[j j]}=\gamma$, sendo $\gamma$ uma medida única que atribui igual interesse para todos os grupos da partição. Alternativamente, pode-se também estimar automaticamente (a partir dos dados) esse fator a partir do nível de interação $\beta[i i \mid j j]$ - Eq. (4.10). Mais precisamente, pode-se fazer com que $\gamma=\beta[i i \mid j j]$.

É sabido que algoritmos para agrupamento de dados impõem/induzem grupos tanto em dados naturalmente agrupados quanto em dados distribuídos aleatoriamente (Jain \& Dubes, 1988a). Este fato é normalmente negligenciado na maioria dos trabalhos da literatura, principalmente pelo fato de que a execução de experimentos controlados permite desprezar tais efeitos aleatórios na maior parte dos casos. No entanto, partições de dados aleatórias podem impactar negativamente, e significativamente, o processo de agrupamento colaborativo, prejudicando outras partições que possuem estrutura de grupos nos dados. Nesses termos, idealmente os conjuntos de dados distribuídos aleatoriamente devem ser explicitamente considerados no modelo de um algoritmo colaborativo. Mais especificamente, assuma que para um sítio de dados específico $(D[j j])$ todos os conjuntos de posições em uma determinada região de interesse do espaço $d$-dimensional sejam igualmente prováveis. Em outras palavras, considere que cada um $\operatorname{dos} N[j j]$ pontos (objetos) foram inseridos aleatoriamente na região de interesse, levandose ao que se denomina hipótese de posições aleatórias (Jain \& Dubes, 1988a). Claramente, as partições obtidas em $D[j j]$ são meramente um artefato encontrado pelo algoritmo de agrupamento de dados, e não representam nenhuma estrutura de grupos encontrada nos dados. Como consequência, os protótipos encontrados nesse sítio são ruins para o processo de agrupamento colaborativo como um todo. Por isso, idealmente deveria ser possível validar cada partição encontrada nos sítios, quantificando-se a aleatoriedade subjacente aos dados de cada sítio. Isso pode ser feito, por exemplo, através da simulação de Monte Carlo. Mais precisamente, inicialmente deve-se estabelecer uma distribuição base de alguma estatística de interesse - e.g., $J$ da função objetivo (2.3) — sob a hipótese nula de nenhuma estrutura (ou aleatoriedade) nos dados. Nesse sentido, uma abordagem simples envolve a geração de $m-1$ conjuntos de dados aleatórios (usando-se distribuição Uniforme) nas mesmas condições do conjunto de dados do sítio $D[j j]$, i.e., com o mesmo número de objetos, mesmo número de atributos e, para cada atributo, respeitando-se seus valores máximos e mínimos. Em seguida, um valor de $J$, considerando- 
se um número fixo de grupos $(c[j j])$ - que poderia ter sido definido a priori ou estimado a partir dos dados de $D[j j]$ - é calculado para cada um dos $m-1$ conjuntos de dados gerados aleatoriamente. Note que se leva em conta, portanto, $m$ conjuntos de dados, ou seja, $m-1$ gerados aleatoriamente mais o disponível em $D[j j]$. Em seguida, um teste estatístico de hipótese é executado. Este teste destina-se a rejeitar a hipótese de aleatoriedade (ou nenhuma estrutura) ao nível de significância $r / m$ se o valor de $J$ do conjunto de dados utilizados no processo de colaboração ( $D[j j]$ neste caso) é um dos menores valores de $r$ obtidos no processo de simulação de Monte Carlo. Para fins de colaboração, não é preciso necessariamente assumir um valor especial para o nível de significância (e.g., $r / m=5 \%$ ), o qual permite decidir se a hipótese nula é rejeitada ou não, ou seja, que decida se a estrutura de grupos encontrada em $D[j j]$ é válida ou não ao nível de significância $r / m$. Em vez disso, com um pouco de abuso de terminologia, pode-se dizer que a significância estatística da partição encontrada em $D[j j]$ pode ser transmitida por meio da variável $n_{i}[j j]$ - Eq. (4.11). Desconsiderando, por enquanto, o fator de interesse, pode-se simplesmente fazer $n_{i}[j j]=(1-r * / m) \sum u_{i j}[j j]$, onde $r *$ é a classificação da partição encontrada em $D[j j]$ de acordo com os $m$ valores obtidos para $J$. Por exemplo, considere uma partição de dados que é a melhor entre 100 partições de dados $(m=100)$, dentre as quais 99 foram obtidas a partir de dados puramente aleatórios. Neste caso, uma partição desse tipo tem o menor valor de $J$ e é classificada como sendo a número $l(r *=1)$. Esta partição pode ser considerada excepcionalmente boa (sob a hipótese nula de nenhuma estrutura com valor $p=0,01$ ) e teria uma significância estatística de $99 \%$, tendo maior peso no processo de agrupamento colaborativo que outra partição induzida a partir de dados aleatórios. Para quantificar o nível de aleatoriedade dos dados, é possível incorporar a significância estatistica no processo de colaboração no fator de interesse, de acordo com a Eq. (4.12):

$$
n_{i}[j j]=\left(1-r^{*} / m\right) \gamma \sum_{j=1}^{N[j j]} u_{i j}[j j]
$$

Considere que o agrupamento colaborativo é realizado em um determinado número de iterações, ou estágios de colaboração, $t_{\max }$. Dado um sítio de dados qualquer (e.g., $\left.D[i i]\right)$, o $t$-ésimo estágio de colaboração ocorrendo nesse sítio pode ser esquematizado pela Figura 4.2. Os resultados obtidos pelo sítio $D[i i]$ ilustrados nessa figura podem ser produzidos por meio do Algoritmo 5. Esse algoritmo essencialmente descreve o algoritmo FCM, exceto pelos passos 5-12 e 15. Nos Passos 5-12 é realizada a inserção dos objetos virtuais (os quais originalmente não estão disponíveis em $D[i i]$ ). Esses objetos virtuais, que serão considerados no processo de agrupamento em $D[i i]$, são os protótipos comunicados por outros sítios de dados. Além disso, cada objeto virtual recebe um peso $\left(w_{K}\right)$, o qual tem como objetivo capturar, por exemplo, a representatividade, interesse ou significância de cada um desses objetos virtuais. Esses pesos são usados para calcular os protótipos de grupos no Passo 15 - Eq. (4.14). É importante enfatizar que o Algoritmo 5 não requer nenhum parâmetro adicional para fins de colaboração além 
dos parâmetros para capturar a representatividade, interesse ou significância, os quais podem ser automaticamente estimados (se assim for desejado). De forma mais ampla, o agrupamento colaborativo ilustrado na Figura 4.2 é realizado para cada sítio de dados para $t=1, \ldots, t_{\text {max }}$. Especificamente, em $t=0$, o algoritmo FCM (Seção 2.3) é executado para cada sítio de dados (sem colaboração). Então, para $t=1, \ldots, t_{\max }$, cada um dos sítios de dados recebem os protótipos e seus pesos comunicados pelos outros sítios, de maneira que o agrupamento em formação seja (em um certo nível) influenciado por tais informações. Para cada sítio de dados, o número de grupos pode ser definido de antemão se este conhecimento de domínio estiver disponível. Uma outra alternativa é estimar esse número automaticamente (e.g., usando o Algoritmo 3 descrito na Seção 2.4.4). Nesse sentido, duas diferentes abordagens podem ser aplicadas. Na primeira, pode-se estimar o número de grupos fuzzy para cada um dos $P$ sítios de dados, $D[1], \ldots, D[P]$, usando apenas as informações disponíveis em seus respectivos conjuntos de $\operatorname{dados} \mathbf{x}_{j}[1] \in \Re^{d} ; j=1, \ldots, N[1] ; \mathbf{x}_{j}[2] \in \Re^{d} ; j=1, \ldots, N[2] ; \ldots ; \mathbf{x}_{j}[P] \in \Re^{d} ; j=1, \ldots, N[P]$, portanto, identificando $c[1], c[2], \ldots, c[P]$ grupos a serem informados como parâmetros fixos ao Algoritmo 5. Neste caso, se os sítios de dados são atualizados constantemente (e.g., como em fluxo contínuo de dados - data streams), qualquer uma dessas estimativas pode ser revista. $\mathrm{Na}$ segunda abordagem, pode-se estimar o número de grupos em cada sítio de dados $D[i i]$, $i i=1, \ldots, P$, levando-se em conta a informação provida pelos outros sítios. Ou seja, pode-se considerar não somente os objetos de $D[i i]$, mas também os protótipos transmitidos (objetos virtuais). Neste caso, o Algoritmo 5 pode ser combinado ao Algoritmo 3. Os novos algoritmos CFCM- $c^{*}$ e CFCM- $\beta_{f}-c^{*}$ levam em conta essa segunda abordagem. Em outras palavras, esses algoritmos são baseados no Algoritmo 3, mas no seu Passo 6, o FCM é substituído pelo CFCM (Algoritmo 5). Além disso, é importante destacar que uma adaptação adicional é necessária: a multiplicação do numerador e do denominador da Silhueta Fuzzy (Simplificada) - Eq. (2.8) pelo peso $\left(w_{K}\right)$ dos objetos. Para computar tais pesos, o Algoritmo CFCM- $c^{*}$ usa a Eq. (4.11), que por considerar apenas a representatividade sugere que tal algoritmo seja adequado para a indução de partições como se os dados estivessem centralizados em um único conjunto de dados. Essa característica sugere que o CFCM- $c^{*}$ é útil para dados provenientes de uma mesma população. Neste caso, é desejável a identificação, em cada um dos sítios de dados, de todos os grupos presentes nos diferentes sítios. O algoritmo CFCM- $\beta_{f}-c^{*}$, por sua vez, computa os pesos dos objetos usando a Eq. (4.12) $\operatorname{com} \gamma=\beta[i i \mid j j]$. Esse $\beta[i i \mid j j]$ é estimado uma única vez e é mantido fixo durante todos os estágios de colaboração - analogamente ao algoritmo CFC- $\beta_{f}$ (Seção 4.3.1). Assim, o algoritmo CFCM- $\beta_{f}-c^{*}$ assume a mesma característica que os algoritmos $\mathrm{CFC}, \mathrm{CFC}-\beta_{d}$, e CFC- $\beta_{f}$ e, portanto, é adequado para cenários nos quais os dados são provenientes de diferentes populações. Note que esses algoritmos permitem o uso de diferentes índices de validade relativos - alternativos à SFS (Seção 2.4.4) — desde que os pesos dos objetos possam ser incorporados no cálculo do índice. 


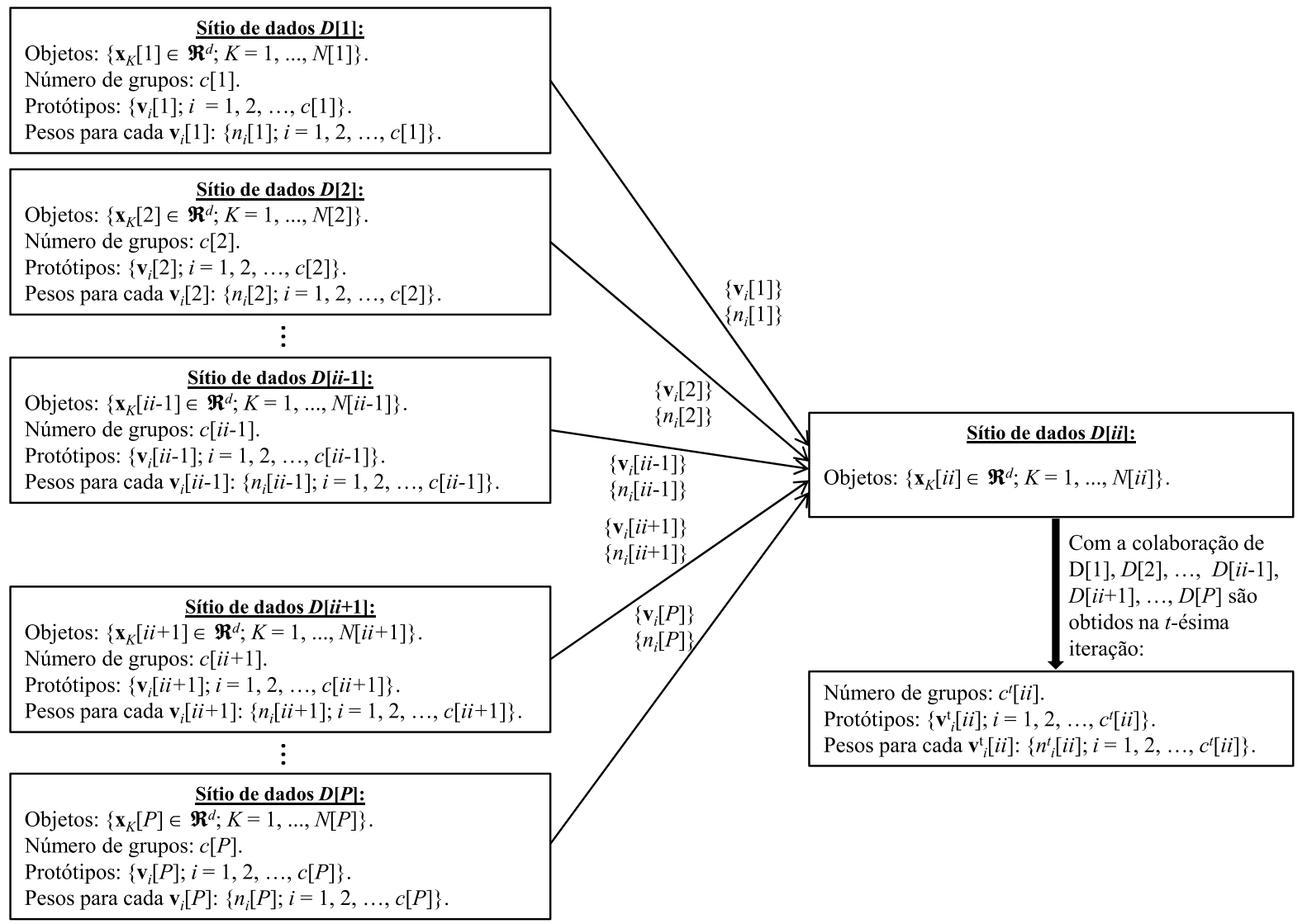

Figura 4.2: Agrupamento colaborativo ocorrendo no sítio de dados $D[i i]$. Todos os outros sítios comunicam seus protótipos e seus respectivos pesos a esse sítio. Como resultado, após o $t$-ésimo estágio de colaboração, tem-se um conjunto de protótipos, $\mathbf{v}_{i}^{t}[i i]$, e seus respectivos pesos, $n_{i}^{t}[i i]\left(i=1,2, \ldots, c^{t}[i i]\right)$, os quais podem ser comunicados a todos os outros sítios no estágio de colaboração $t+1$. Esse mesmo esquema também ocorre nos sítios $D[1], D[2], \ldots$, $D[i i-1], D[i i+1], \ldots, D[P]$. 


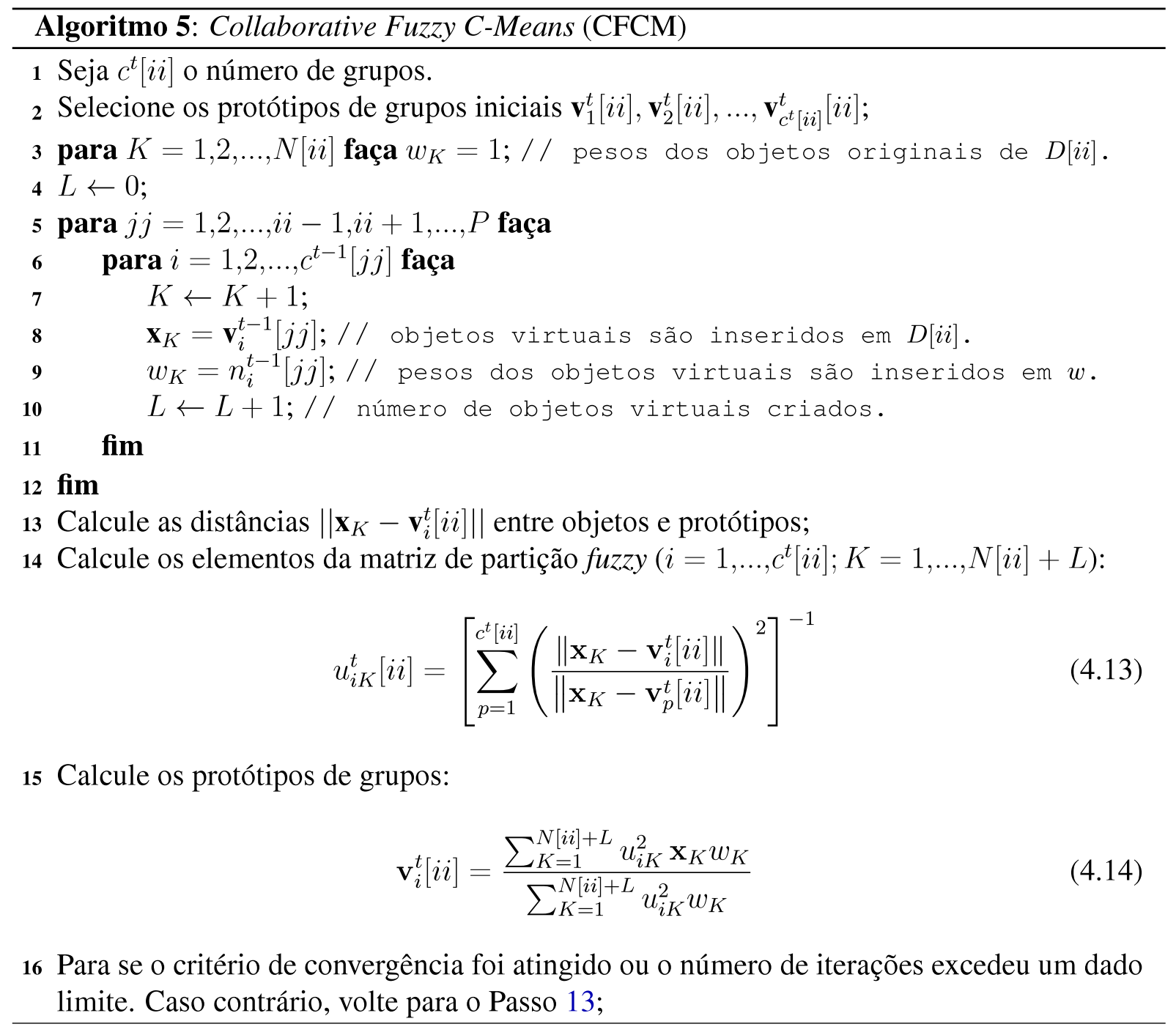




\subsubsection{Algoritmo PFCM- $c^{*}$ — Número de Grupos Variável}

O algoritmo Parallel Fuzzy C-Means (PFCM) (Rahimi et al., 2004) é uma extensão do algoritmo FCM (Seção 2.3) capaz de processar os dados de forma paralela. Esse algoritmo encontra, para um número fixo de grupos $(c)$, uma partição fuzzy que é precisamente a mesma que seria encontrada se todos os dados (originalmente distribuídos em diferentes sítios) estivessem centralizados em um único local. Devido a essa característica e a forma como compartilha as informações entre os sítios, o algoritmo PFCM é potencialmente útil para agrupamento fuzzy colaborativo, especialmente em cenários nos quais se assume que os dados distribuídos são de uma mesma população. Essencialmente, esse algoritmo agrupa dados distribuídos por meio da comunicação de dois termos parciais entre os sítios de dados. Esses termos são vetores que correspondem aos numeradores e denominadores parciais usados na Eq. (2.5) do algoritmo FCM (Seção 2.3). A agregação desses vetores (a partir da soma de seus elementos) resulta em um vetor de protótipos que é transmitido a todos os sítios de dados. Assumindo esses protótipos como protótipos iniciais, os dados de cada um dos sítios podem ser reagrupados (baseando-se no algoritmo FCM), produzindo-se, assim, um novo conjunto de termos parciais, os quais podem ser novamente agregados para serem usados em uma próxima iteração do algoritmo (i.e., num próximo estágio de colaboração). Esse procedimento é realizado até que os protótipos não se alterem significativamente em duas iterações consecutivas. Conforme já discutido, o algoritmo PFCM requer que o número de grupos, $c$, seja informado pelo usuário. Com o objetivo de superar essa limitação, um novo algoritmo foi desenvolvido, o qual essencialmente substitui o FCM pelo PFCM no Passo 6 do Algoritmo 3. Além disso, também é empregada a versão distribuída da SFS (Seção 2.4.4), a qual necessita apenas das distâncias entre objetos e protótipos. Dado que, para o PFCM, os protótipos são os mesmos para todos os sítios de dados, pode-se facilmente calcular as silhuetas (parciais) para cada sítio - usando a Eq. (2.8) - e agregá-las de forma a obter a silhueta global (exata, sem qualquer aproximação) ${ }^{2}$. Esse novo algoritmo capaz de automaticamente estimar o número de grupos é aqui chamado de PFCM- $c^{*}$.

\subsection{Seleção dos Algoritmos}

Na prática, normalmente não é uma tarefa fácil selecionar, dentre vários algoritmos, o algoritmo mais apropriado para uma aplicação em particular. Para auxiliar na escolha do algoritmo para agrupamento fuzzy colaborativo potencialmente mais apropriado para uma determinada aplicação, dois fatores sao bastante relevantes: (i) suposições sobre os dados (i.e., dados que são de uma mesma população ou de diferentes populações); e (ii) a dificuldade em se definir parâmetros fundamentais dos algoritmos. Nesse sentido, a Figura 4.3 ilustra uma árvore de decisão que visa auxiliar o usuário na escolha do algoritmo mais adequado para o problema que se tem em mãos. Em particular, os algoritmos CFCM- $c^{*}$ e CFCM- $\beta_{f}-c^{*}$ são capazes de estimar automaticamente o número de grupos para cada um dos sítios de dados. Claramente essa

\footnotetext{
${ }^{2}$ Note que existem outros critérios de validade relativos para os quais essa propriedade também é válida - e.g. (Xie \& Beni, 1991).
} 
característica é mais interessante para os algoritmos do Cenário 1, no qual assume-se que os dados distribuídos são de diferentes populações. Ao assumir que os dados são de uma mesma população (Cenário 2), o algoritmo $\mathrm{PFCM}-c^{*}$ pode ser preferível ao $\mathrm{CFCM}-c^{*}$ se o número de grupos é desconhecido, pois o PFCM- $c^{*}$ está em maior conformidade com as suposições sobre os dados - conforme será ilustrado por meio dos resultados experimentais reportados na Seção 5.3.2. No Cenário 1, se o número de grupos é conhecido a priori, três algoritmos podem ser usados. Contudo, é importante notar que o algoritmo CFC requer que o usuário defina o nível de interação $\beta$, o que pode ser uma tarefa difícil na prática - e.g., ver Seção 5.2.1. Os algoritmos CFC- $\beta_{d}$ e CFC- $\beta_{f}$, por sua vez, estimam esse parâmetro automaticamente a partir dos dados sendo, portanto, em princípio mais recomendados. Finalmente, as Tabelas 4.1, 4.2 e 4.3 sumarizam, respectivamente, as análises das complexidades de tempo, espaço e comunicação. Levando-se em conta as complexidades computacionais de cada algoritmo, pode-se concluir que: (i) para o Cenário 1, com número de grupos conhecido, tem-se três alternativas de algoritmos, sendo que todos eles apresentam complexidades computacionais idênticas; (ii) Para o Cenário 2 e assumindo-se que o número de grupos é desconhecido, pode-se perceber que há diferenças entre os algoritmos estudados. Em particular, o $\mathrm{CFCM}-c^{*}$ possui uma complexidade de tempo assintótica maior do que o PFCM- $c^{*}$. 


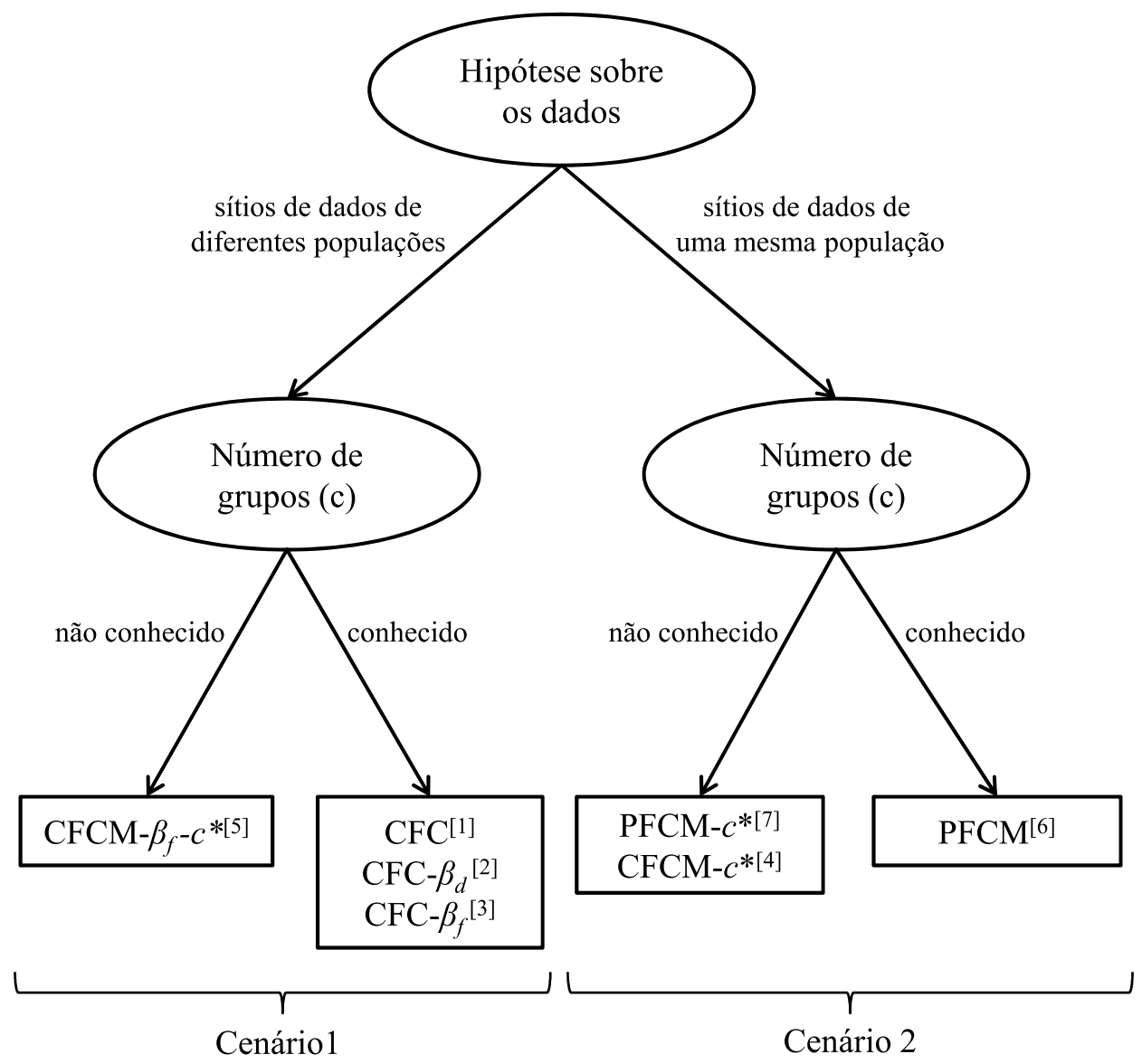

Figura 4.3: Árvore de decisão para a escolha dos algoritmos.

[1] CFC (Pedrycz \& Rai, 2008) - Seção 4.2.

[2] CFC- $\beta_{d}$ - Seção 4.3.1.

[3] CFC- $\beta_{f}$ - Seção 4.3.1.

[4] CFCM- $c^{*}$ - Seção 4.3.2.

[5] CFCM- $\beta_{f}-c^{*}$ - Seção 4.3.2.

[6] PFCM (Rahimi et al., 2004) — Seção 3.1.2.

[7] PFCM-c* — Seção 4.3.3. 
Tabela 4.1: Complexidade de tempo dos algoritmos considerando o mesmo número de objetos $(N)$ e grupos $(c)$ para todos os sítios de dados $-n_{t}$ é o número de iterações, $t$ o número de estágios de colaboração e $n_{p}$ o número de diferentes partições geradas.

\begin{tabular}{|c|c|}
\hline Algoritmo & Complexidade de Tempo \\
\hline $\mathrm{CFC}^{[1]}$ & $O\left(\left(\left(c^{2} \cdot N \cdot d\right)+\left(c^{2} \cdot N \cdot P\right)+(c \cdot N \cdot d \cdot P)\right) \cdot\left(n_{t} \cdot t \cdot P\right)\right)$ \\
\hline CFC $-\beta_{d}{ }^{22]}$ & $O\left(\left(\left(c^{2} \cdot N \cdot d\right)+\left(c^{2} \cdot N \cdot P\right)+(c \cdot N \cdot d \cdot P)\right) \cdot\left(n_{t} \cdot t \cdot P\right)\right)$ \\
\hline $\mathrm{CFC}-\beta_{f}^{[3]}$ & $O\left(\left(\left(c^{2} \cdot N \cdot d\right)+\left(c^{2} \cdot N \cdot P\right)+(c \cdot N \cdot d \cdot P)\right) \cdot\left(n_{t} \cdot t \cdot P\right)\right)$ \\
\hline $\mathrm{CFCM}-c^{*[4]}$ & $O\left(\left(\left(c_{\max }{ }^{4} \cdot d \cdot P \cdot n_{t} \cdot n_{p}\right)+\left(c_{\max }{ }^{3} \cdot N \cdot d \cdot n_{t} \cdot n_{p}\right)\right) \cdot(t \cdot P)\right)$ \\
\hline CFCM- $\beta_{f}-c^{*[5]}$ & $O\left(\left(\left(c_{\max }{ }^{4} \cdot d \cdot P \cdot n_{t} \cdot n_{p}\right)+\left(c_{\max }{ }^{3} \cdot N \cdot d \cdot n_{t} \cdot n_{p}\right)+\left(c_{\max } \cdot N \cdot d \cdot P\right)\right) \cdot(t \cdot P)\right)$ \\
\hline $\mathrm{PFCM}^{[6]}$ & $O\left(c^{2} \cdot N \cdot d \cdot P \cdot n_{t}\right)$ \\
\hline $\mathrm{PFCM}-c^{*[7]}$ & $O\left(c_{\max }{ }^{3} \cdot N \cdot d \cdot P \cdot n_{t} \cdot n_{p}\right)$ \\
\hline
\end{tabular}

Tabela 4.2: Complexidade de espaço dos algoritmos considerando um único sítio de dados com $N$ objetos e $c$ grupos.

\begin{tabular}{|c|c|}
\hline Algoritmo & Complexidade de Espaço \\
\hline $\mathrm{CFC}^{[1]}$ & $O((c \cdot N \cdot P)+(c \cdot d \cdot P))$ \\
\hline $\mathrm{CFC}-\beta_{d}{ }^{[2]}$ & $O((c \cdot N \cdot P)+(c \cdot d \cdot P))$ \\
\hline $\mathrm{CFC}-\beta_{f}{ }^{[3]}$ & $O((c \cdot N \cdot P)+(c \cdot d \cdot P))$ \\
\hline $\mathrm{CFCM}-c^{*[4]}$ & $O\left(\left(c_{\max }{ }^{2} \cdot P\right)+\left(c_{\max } \cdot d \cdot P\right)+\left(c_{\max } \cdot N\right)\right)$ \\
\hline CFCM- $\beta_{f}-c^{*[5]}$ & $O\left(\left(c_{\max }{ }^{2} \cdot P\right)+\left(c_{\max } \cdot d \cdot P\right)+\left(c_{\max } \cdot N\right)\right)$ \\
\hline $\mathrm{PFCM}^{[6]}$ & $O((c \cdot N \cdot P)+(c \cdot d))$ \\
\hline PFCM- $c^{*[7]}$ & $O\left(\left(c_{\max } \cdot N \cdot P\right)+\left(c_{\max } \cdot d\right)\right)$ \\
\hline
\end{tabular}

Tabela 4.3: Complexidade de comunicação dos algoritmos considerando o mesmo número de objetos $(N)$ e grupos $(c)$ para todos os sítios de dados $-n_{t}$ é o número de iterações, $t$ o número de estágios de colaboração e $n_{p}$ o número de diferentes partições geradas.

\begin{tabular}{|c|c|}
\hline Algoritmo & Complexidade de Comunicação \\
\hline $\mathrm{CFC}^{[1]}$ & $O(c \cdot d \cdot t \cdot P)$ \\
\hline $\mathrm{CFC}-\beta_{d}[2]$ & $O(c \cdot d \cdot t \cdot P)$ \\
\hline $\mathrm{CFC}-\beta_{f}[3]$ & $O(c \cdot d \cdot t \cdot P)$ \\
\hline $\mathrm{CFCM}^{[3]} c^{*[4]}$ & $O\left(c_{\max } \cdot d \cdot t \cdot P\right)$ \\
\hline $\mathrm{CFCM}-\beta_{f}-c^{*[5]}$ & $O\left(c_{\max } \cdot d \cdot t \cdot P\right)$ \\
\hline $\mathrm{PFCM}^{[6]}$ & $O\left(c \cdot d \cdot P \cdot n_{t}\right)$ \\
\hline PFCM- $c^{*[7]}$ & $O\left(c_{\max }{ }^{2} \cdot d \cdot P \cdot n_{t} \cdot n_{p}\right)$ \\
\hline
\end{tabular}




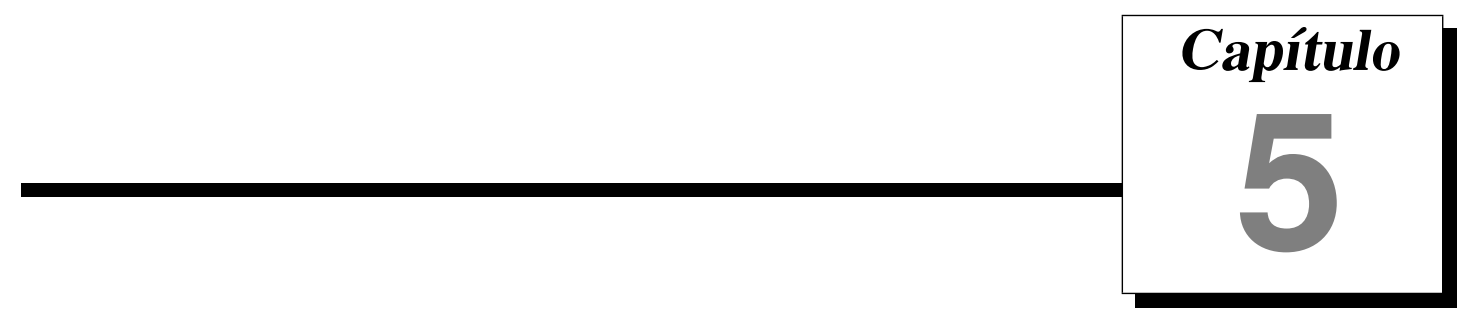

\section{Avaliação Experimental}

Nesse capítulo são apresentados e discutidos os experimentos realizados nesse trabalho. Em particular, a Seção 5.1 descreve os conjuntos de dados utilizados. A Seção 5.2, por sua vez, envolve uma análise experimental dos algoritmos CFC (Seção 4.2), CFC- $\beta_{d}$ e CFC- $\beta_{f}$ (Seção 4.3.1). Esses algoritmos foram estudados e comparados levando-se em conta a dificuldade em se determinar alguns dos parâmetros requeridos (nível de interação $\beta$ e/ou número de grupos $c$ ). $\mathrm{Na}$ Seção 5.3 são abordados exemplos ilustrativos que destacam as diferenças de desempenho entre os algoritmos, levando-se em conta dois cenários específicos de aplicação baseados na árvore de decisão da Figura 4.3. Esses cenários consideram as suposições sobre os dados (i.e., dados que são de uma mesma população ou de diferentes populações). Além disso, são apresentados exemplos que ilustram o comportamento dos algoritmos na presença de dados aleatórios e de dados multivariados. Por fim, na Seção 5.4, são apresentadas as considerações finais sobre os experimentos realizados.

\subsection{Conjuntos de Dados}

Seis conjuntos de dados foram utilizados nos experimentos (um desses consiste de dados reais e os outros cinco de dados sintéticos). O conjunto de dados reais foi obtido a partir do repositório da University of California, Irvine (Asuncion \& Newman, 2007). Esse conjunto, chamado de Wisconsin Breast Cancer, consiste de 683 objetos descritos por nove atributos. Cada um desses objetos possui uma entre duas classes possíveis (benigno ou maligno), havendo 444 objetos da classe benigno e 239 da classe maligno. Esses dados são de amostras coletadas periodicamente de casos clínicos da University of Wisconsin Hospitals. Nesse sentido, esse conjunto reflete o agrupamento cronológico dos dados. Os conjuntos de dados sintéticos, por sua vez, são descritos a seguir: 
Sin1: Inspirado no trabalho de Pedrycz \& Rai (2008) este conjunto é composto por cinco sítios de dados bi-dimensionais $(D[1], D[2], \ldots, D[5])$. Cada sítio contém 600 objetos, divididos em 3 grupos gerados segundo uma mistura de três distribuições Gaussianas, as quais são descritas pelos seguintes vetores médios representativos de seus centros:

$$
\begin{aligned}
& D[1]: \mathbf{v}_{1}=[4,0 ; 4,5], \mathbf{v}_{2}=[8,5 ; 10,0], \mathbf{v}_{3}=[10,0 ; 4,0] ; \\
& D[2]: \mathbf{v}_{1}=[4,5 ; 4,5], \mathbf{v}_{2}=[10,5 ; 4,0], \mathbf{v}_{3}=[10,5 ; 10,0] ; \\
& D[3]: \mathbf{v}_{1}=[4,5 ; 6,0], \mathbf{v}_{2}=[7,0 ; 9,0], \mathbf{v}_{3}=[10,0 ; 6,0] ; \\
& D[4]: \mathbf{v}_{1}=[6,0 ; 4,0], \mathbf{v}_{2}=[6,0 ; 10,0], \mathbf{v}_{3}=[10,0 ; 10,0] ; \\
& D[5]: \mathbf{v}_{1}=[4,0 ; 8,0], \mathbf{v}_{2}=[8,0 ; 8,0], \mathbf{v}_{3}=[10,0 ; 5,0] ;
\end{aligned}
$$

Sin2: Esse conjunto é composto por dois sítios de dados bi-dimensionais ( $D[1], D[2])$. Cada sítio de dados contém 200 objetos, divididos em 2 grupos gerados de acordo com uma mistura de duas distribuições Gaussianas, as quais são descritas pelos seguintes vetores médios representativos de seus centros:

$$
\begin{aligned}
& D[1]: \mathbf{v}_{1}=[3,0 ; 2,0], \mathbf{v}_{2}=[8,0 ; 6,0] ; \\
& D[2]: \mathbf{v}_{1}=[6,0 ; 3,0], \mathbf{v}_{2}=[11,0 ; 7,0] ;
\end{aligned}
$$

Sin3: Conjunto composto de dois sítios de dados bi-dimensionais $(D[1], D[2])$. Cada um com 30 objetos, divididos em 3 grupos gerados segundo uma mistura de três distribuições Gaussianas, descritas pelos vetores médios representativos de seus centros:

$$
\begin{aligned}
& D[1]: \mathbf{v}_{1}=[2,0 ; 2,0], \mathbf{v}_{2}=[2,0 ; 5,0], \mathbf{v}_{3}=[5,0 ; 5,0] ; \\
& D[2]: \mathbf{v}_{1}=[5,0 ; 2,0], \mathbf{v}_{2}=[5,0 ; 5,0], \mathbf{v}_{3}=[2,0 ; 2,0] ;
\end{aligned}
$$

Sin4: Composto por dois sítios de dados bi-dimensionais $(D[1]$ e $D[2])$. Cada um contendo 400 objetos, divididos em 2 grupos gerados a partir de uma mistura de duas distribuições Gaussianas descritas pelos vetores médios representativos de seus centros:

$$
\begin{aligned}
& D[1]: \mathbf{v}_{1}=[10,0 ; 4,0], \mathbf{v}_{2}=[4,0 ; 10,0] ; \\
& D[2]: \mathbf{v}_{1}=[10,0 ; 10,0], \mathbf{v}_{2}=[4,0 ; 4,0] ;
\end{aligned}
$$

Sin5: Composto por dois sítios de dados bi-dimensionais ( $D[1]$ e $D[2])$. Cada sítio com 600 objetos, divididos em 3 grupos gerados a partir de uma mistura de três distribuições Gaussianas descritas pelos seguintes vetores médios representativos de seus centros:

$$
\begin{aligned}
& D[1]: \mathbf{v}_{1}=[10,0 ; 4,0], \mathbf{v}_{2}=[4,0 ; 4,5], \mathbf{v}_{3}=[8,5 ; 10,0] ; \\
& D[2]: \mathbf{v}_{1}=[10,0 ; 10,0], \mathbf{v}_{2}=[6,0 ; 4,0], \mathbf{v}_{3}=[6,0 ; 10,0] ;
\end{aligned}
$$

Para todos esses conjuntos de dados foi utilizada a matriz de covariâncias $\left[\begin{array}{ll}1 & 0 \\ 0 & 1\end{array}\right]$ para gerar os grupos em cada um dos sítios de dados, com exceção do conjunto de dados $\operatorname{Sin} 3$, para o qual foi usado a matriz de covariâncias $\left[\begin{array}{cc}0,3 & 0 \\ 0 & 0,3\end{array}\right]$. 


\subsection{Análise dos Algoritmos CFC, CFC- $\beta_{d}$ e CFC- $\beta_{f}$}

Nessa seção são apresentadas as análises realizadas com os algoritmos CFC (Seção 4.2), CFC- $\beta_{d}$ e CFC- $\beta_{f}$ (Seção 4.3.1). Resumidamente, na Seção 5.2.1, é avaliado um procedimento iterativo sugerido pelos autores do artigo (Pedrycz \& Rai, 2008) para se estimar o nível de interação $\beta$ no algoritmo CFC. Na Seção 5.2.2, por sua vez, são abordados experimentos comparativos entre esse algoritmo (CFC) e os algoritmos CFC- $\beta_{d}$ e CFC- $\beta_{f}$ (levando-se em conta que esses dois últimos estimam automaticamente os níveis de interação entre os sítios). Por fim, a Seção 5.2.3 envolve uma análise experimental que enfatiza a limitação desses algoritmos ao fixarem um mesmo número de grupos $(c)$ para todos os sítios de dados. Em especial, ao se considerar que os dados distribuídos nos diferentes sítios são provenientes de diferentes populações.

\subsubsection{Nível de Interação $\beta$ Estimado Iterativamente - Algoritmo CFC}

Os autores do artigo (Pedrycz \& Rai, 2008) sugeriram um procedimento iterativo baseado na minimização do índice de qualidade $W$ (Seção 4.2) para se estimar o nível de interação $\beta$ no algoritmo CFC. A avaliação desse procedimento, bem como a análise do processo de colaboração realizado pelo algoritmo nos conjuntos de dados $\operatorname{Sin} 1$ e $\operatorname{Sin} 2$, são o foco dos experimentos reportados nessa seção. O procedimento iterativo para estimação do nível de interação $\beta$ envolve várias execuções do algoritmo $\mathrm{CFC}$, cada qual com um valor específico de $\beta$. $\mathrm{O}$ índice de qualidade $W$ é computado para cada uma dessas execuções e o valor de $\beta$ que produz o menor valor de $W$ é selecionado como sendo o $\beta$ ótimo (ver Seção 4.2). Inicialmente, foi reproduzido o experimento reportado em (Pedrycz \& Rai, 2008) para o conjunto de da$\operatorname{dos} \operatorname{Sin} 1 \operatorname{com} c=3$ (que corresponde ao número de componentes da mistura de Gaussianas). A Figura 5.1 ilustra o gráfico com os valores de $W_{F}$ ( $W$ final, minimizado após o processo de colaboração) para cada execução do algoritmo CFC (cada qual com um valor particular de $\beta=\{0,001 ; 0,05 ; 0,10 ; \ldots ; 1\})$. Nesse gráfico, nota-se que para $\beta>0,10$ os valores de $W_{F}$ decresceram monotonicamente $\operatorname{com} \beta$. Em particular, $W_{F}$ teve uma acentuada diminuição entre $\beta=0,10$ e $\beta=0,25$ e, para valores de $\beta$ superiores a 0,25 , ocorreram melhorias menos significativas. Em (Pedrycz \& Rai, 2008) os autores assumiram $\beta=0,25$ como sendo o $\beta$ ótimo, o que poderia ser naturalmente considerado ao se levar em conta que para $\beta>0,25$ os valores de $W_{F}$ não diminuem muito (ver Figura 5.1). No entanto, é importante salientar que com $\beta=1$ alcançou-se $W_{F}=1,69$, que é aproximadamente $15 \%$ menor do que $W_{F}=2,04$ obtido com $\beta=0,25$. Assim, assumindo-se que o nível de interação $\beta$ ótimo é o que minimiza $W$, poderia ser mais adequado (para Sin1), em princípio, selecionar $\beta=1$ como sendo o valor ótimo.

Visando reproduzir o experimento reportado em (Pedrycz \& Rai, 2008), fixou-se $\beta=0,25$. A Tabela 5.1 indica as coordenadas $(x, y)$ dos protótipos obtidos. Na primeira coluna estão os protótipos gerados após a Fase Inicial do algoritmo (sem colaboração) e na segunda coluna estão os protótipos gerados após a Fase Colaborativa (20 estágios de colaboração). Claramente nota-se que os protótipos não mudaram muito após o processo de colaboração, sugerindo que 
o algoritmo CFC realizou apenas um ajuste fino em função das informações comunicadas entre os sítios de dados. A Figura 5.2 ilustra o gráfico do comportamento de $W$, que diminuiu de 4,39 para 2,04 durante os 20 estágios de colaboração. Esses valores apresentaram, respectivamente, uma diferença de apenas $0,44 \%$ e $0,30 \%$ em relação aos valores publicados em (Pedrycz \& Rai, 2008). Nesse sentido, pode-se sugerir que o comportamento do algoritmo CFC neste experimento aproximou-se bastante do comportamento reportado no experimento da Seção 9.1 do artigo em questão.

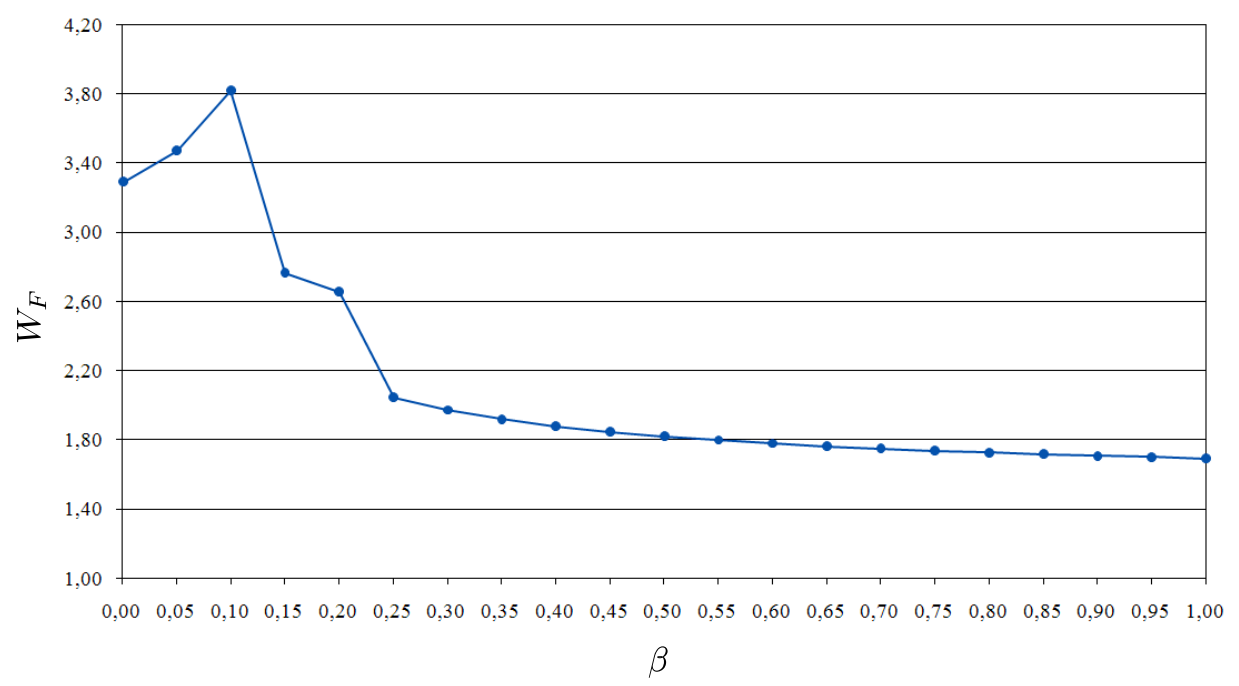

Figura 5.1: Valores de $W_{F}$ de acordo $\operatorname{com} \beta$ informado ao algoritmo CFC - Conjunto Sin1.

Tabela 5.1: Protótipos obtidos com o algoritmo CFC $(\beta=0,25)$ - Conjunto Sin1.

\begin{tabular}{|c|c|c|c|}
\hline \multicolumn{3}{|c|}{ Após Fase Inicial } & \multicolumn{1}{|c|}{ Após Fase Colaborativa } \\
\hline \multicolumn{4}{|c|}{$D[1]$} \\
\hline 8,53 & 10,07 & 8,52 & 10,05 \\
\hline 3,94 & 4,40 & 3,93 & 4,50 \\
\hline 10,00 & 4,02 & 9,95 & 4,01 \\
\hline \multicolumn{3}{|c|}{$D[2]$} \\
\hline 4,52 & 4,46 & 4,45 & 4,56 \\
\hline 10,51 & 3,82 & 10,46 & 3,88 \\
\hline 10,54 & 10,12 & 10,46 & 10,16 \\
\hline \multicolumn{4}{|c|}{$D[3]$} \\
\hline 10,09 & 5,87 & 10,04 & 5,65 \\
\hline 4,35 & 5,94 & 4,41 & 6,06 \\
\hline 7,09 & 9,05 & 7,41 & 8,97 \\
\hline \multicolumn{4}{|c|}{$D[4]$} \\
\hline 6,07 & 3,89 & 5,41 & 5,39 \\
\hline 9,96 & 10,07 & 8,44 & 10,14 \\
\hline 5,89 & 9,98 & 6,89 & 3,88 \\
\hline \multicolumn{4}{|c|}{$D[5]$} \\
\hline 8,11 & 8,18 & 7,96 & 8,42 \\
\hline 10,07 & 4,98 & 9,98 & 5,02 \\
\hline 3,99 & 8,14 & 4,00 & 7,91 \\
\hline
\end{tabular}




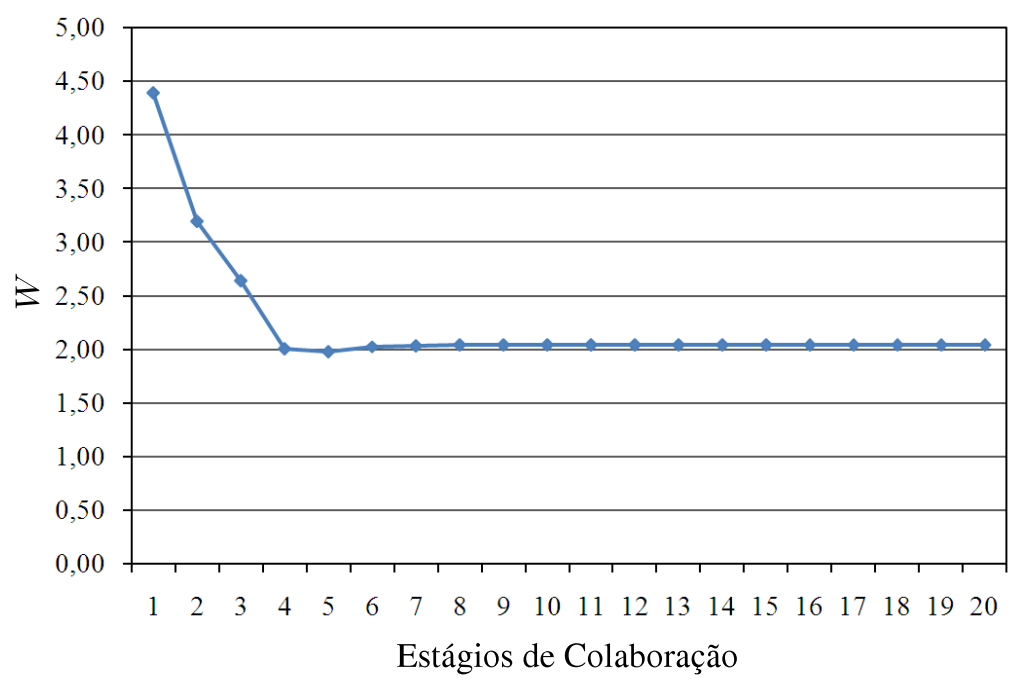

Figura 5.2: Comportamento de $W$ - Algoritmo CFC $(\beta=0,25)$ - Conjunto Sin1.

Experimentos análogos foram realizados usando-se o conjunto de dados Sin2. A Figura 5.3 apresenta o gráfico com os valores de $W_{F}$ para os diferentes valores de $\beta$ fornecidos ao algoritmo CFC ( $c=2$ ). Por este gráfico pode-se constatar que $W_{F}$ diminuiu com o aumento de $\beta$, similarmente ao experimento anterior (ver Figura 5.1). De fato, este comportamento é esperado, pois quanto maior o valor de $\beta$, mais forte é a colaboração entre os sítios de dados, o que tende a tornar as estruturas dos sítios mais parecidas entre si (em função de ajustes nos protótipos). Como consequência, os valores de $W$ tendem a ser menores. Contudo, vale ressaltar que não é uma alternativa completamente viável e útil informar valores altos de $\beta$ para arbitrariamente se alcançar valores menores de $W$. Por exemplo, uma forte colaboração pode não ser útil quando os sítios de dados forem muito diferentes. Nesse caso, levando-se em conta que o algoritmo CFC assume que os dados são provenientes de diferentes populações, uma forte colaboração poderia afetar negativamente o processo de colaboração. Nesse sentido, uma idéia interessante seria estimar o nível de interação com base nos próprios dados, como é feito nos algoritmos CFC- $\beta_{d}$ e CFC- $\beta_{f}$ (abordados na Seção 5.2.2).

A Figura 5.4 apresenta o gráfico do comportamento de $W$ para $\beta=0,95$. O valor mínimo de $W(0,20)$ foi alcançado no segundo estágio de colaboração. A Figura 5.5 ilustra os grupos obtidos, sendo que pi indica a posição inicial dos protótipos (sem colaboração — após a Fase Inicial) e $p f$ a posição final (após a Fase Colaborativa - 15 estágios de colaboração). Notase nessas partições que os protótipos se reposicionaram como consequência do processo de colaboração realizado pelo algoritmo. No entanto, apesar do valor do índice $W$ ter diminuído aproximadamente $20 \%$, apenas pequenas mudanças (na direção esperada) foram observadas nesses protótipos. Em outras palavras, mesmo com uma forte colaboração $(\beta=0,95)$, não ocorreram mudanças significativas nos grupos em cada um dos sítios de dados. 


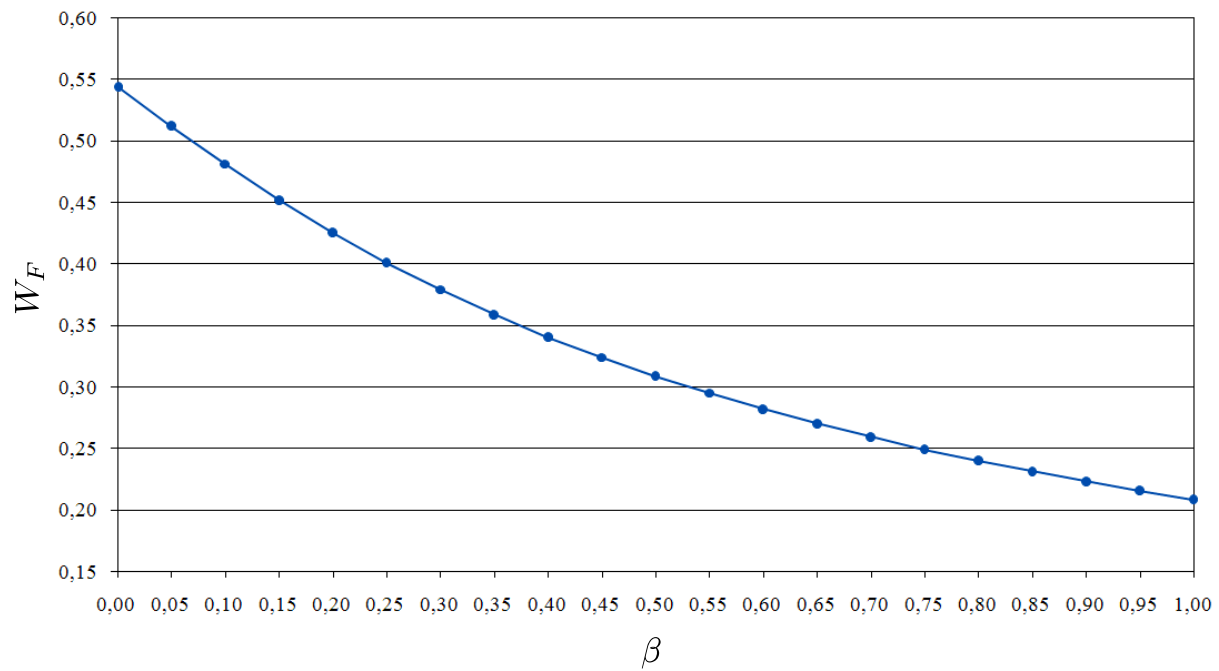

Figura 5.3: Valores de $W_{F}$ de acordo $\operatorname{com} \beta$ informado ao algoritmo CFC - Conjunto Sin2.

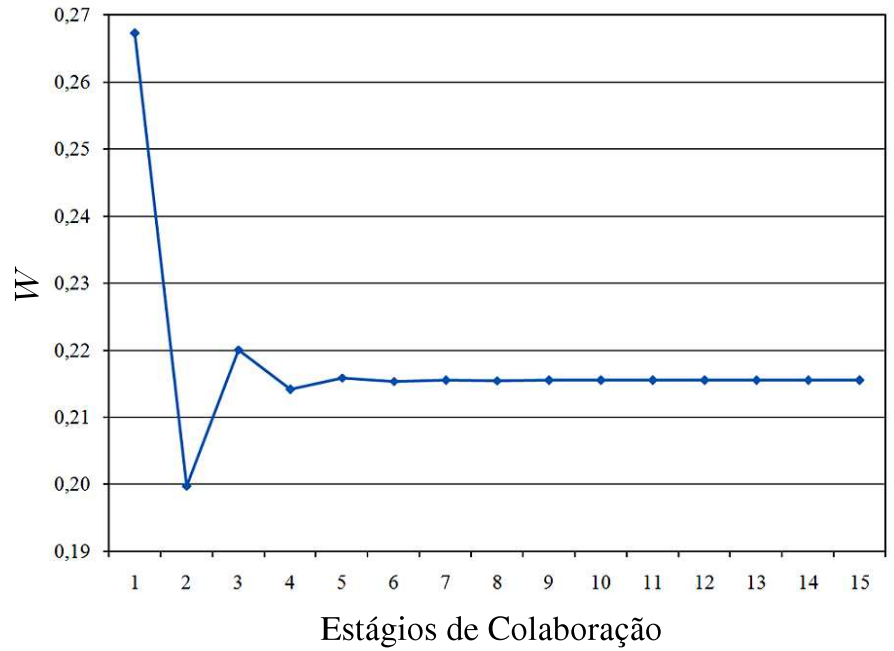

Figura 5.4: Comportamento de $W$ - Algoritmo CFC $(\beta=0,95)$ — Conjunto Sin2.
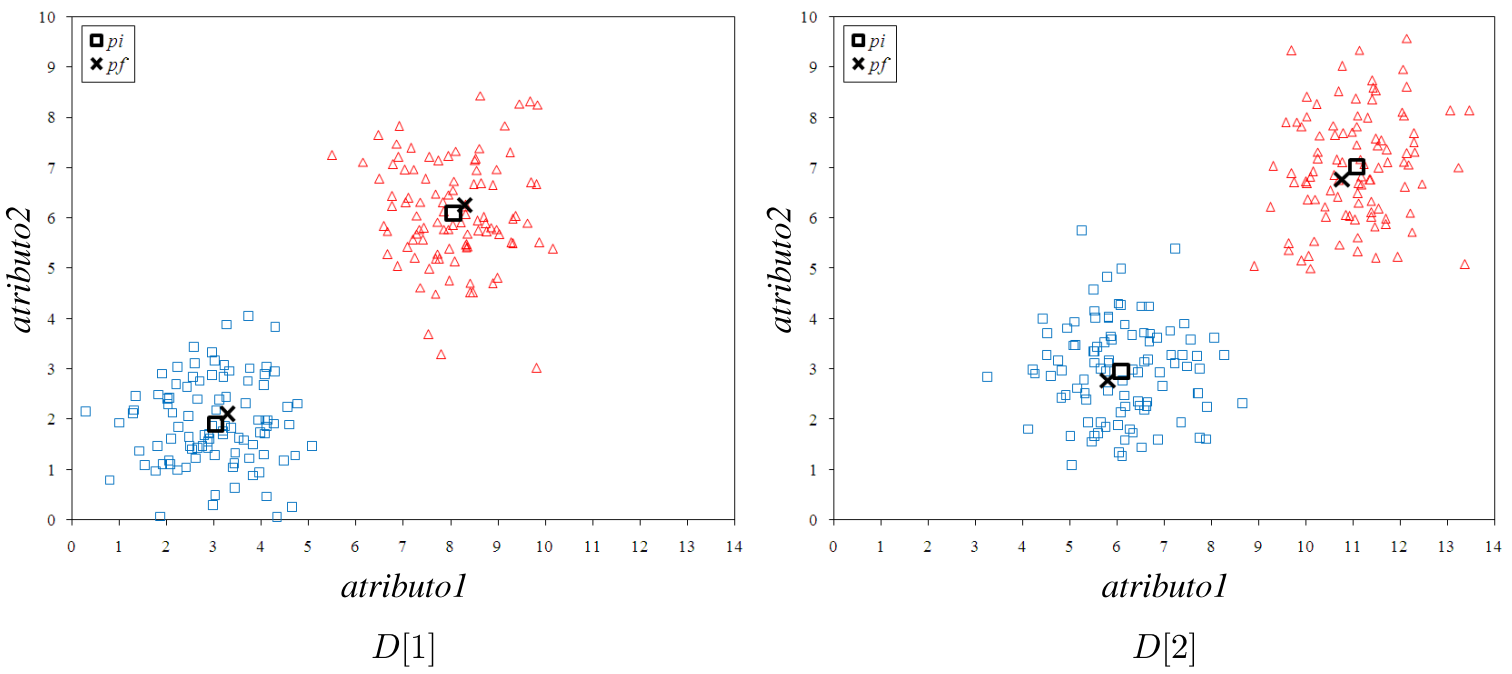

Figura 5.5: Grupos obtidos com o algoritmo CFC — Conjunto Sin2. 


\subsubsection{Nível de Interação $\beta$ Estimado Automaticamente - Algoritmos CFC- $\beta_{d}$ e CFC- $\beta_{f}$}

Nesta seção são apresentados os experimentos realizados com os algoritmos CFC- $\beta_{d}$ e CFC$\beta_{f}$ (Seção 4.3.1). Esses algoritmos estimam automaticamente (a partir dos dados) um nível de interação $\beta$ específico para cada par de sítios de dados. Em particular, os experimentos reportados envolvem um estudo comparativo desses algoritmos em relação ao algoritmo CFC (analisado na seção anterior). A Figura 5.6 ilustra o comportamento do índice $W$ para o algoritmo CFC- $\beta_{d}$ no conjunto de dados Sin1. Nota-se nesse gráfico que o valor de $W$ diminuiu de 4,12 para 1,91. Esses valores são menores do que os obtidos com o algoritmo CFC nesse mesmo conjunto de dados (respectivamente, 4,39 e 2,04). Neste caso, assumindo-se que valores mais baixos de $W$ indicam uma maior consistência entre os sítios de dados (Seção 4.2), pode-se considerar que o uso da Eq. (4.10) para se estimar o nível de interação $\beta[i i \mid j j], i i, j j=1, \ldots, P$, entre os sítios de dados melhorou o processo de colaboração. A Tabela 5.2 indica as coordenadas $(x, y)$ dos protótipos após a Fase Inicial do algoritmo CFC- $\beta_{d}$ (sem colaboração) e após a Fase Colaborativa (20 estágios de colaboração). Nota-se que os protótipos dessa tabela são muito parecidos com aqueles reportados na Tabela 5.1 (referente ao algoritmo CFC). Esta constatação sugere que o algoritmo CFC- $\beta_{d}$ manteve as características do algoritmo CFC, mas com a vantagem de não ser necessária a informação do parâmetro $\beta$.

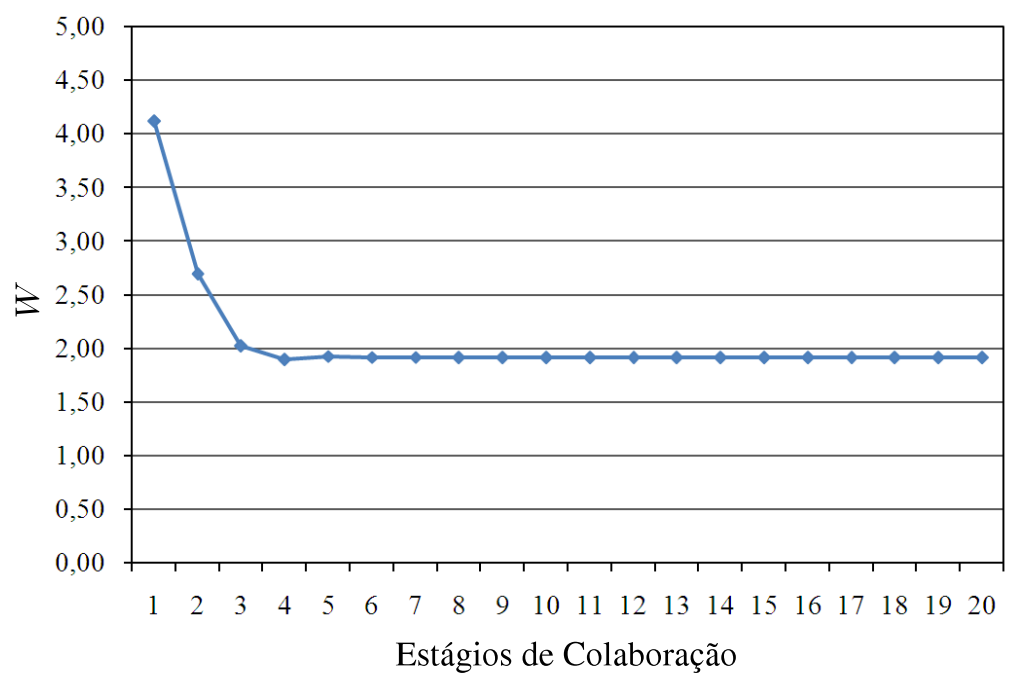

Figura 5.6: Comportamento de $W$ - Algoritmo CFC- $\beta_{d}$ - Conjunto Sin1. 
Tabela 5.2: Protótipos obtidos com o algoritmo CFC- $\beta_{d}$ - Conjunto Sin1.

\begin{tabular}{|c|c|c|c|}
\hline \multicolumn{2}{|c|}{ Após Fase Inicial } & \multicolumn{2}{|c|}{ Após Fase Colaborativa } \\
\hline \multicolumn{2}{|c|}{$D[1$} & 1] & \\
\hline 8,53 & 10,07 & 8,53 & 10,05 \\
\hline 3,94 & 4,40 & 3,93 & 4,49 \\
\hline 10,00 & 4,02 & 9,96 & 4,01 \\
\hline \multicolumn{4}{|c|}{$D[2]$} \\
\hline 4,52 & 4,46 & 4,46 & 4,54 \\
\hline 10,51 & 3,82 & 10,47 & 3,87 \\
\hline 10,54 & 10,12 & 10,47 & 10,15 \\
\hline \multicolumn{4}{|c|}{$D[3]$} \\
\hline 10,09 & 5,87 & 10,02 & 5,53 \\
\hline 4,35 & 5,94 & 4,43 & 6,09 \\
\hline 7,09 & 9,05 & 7,58 & 8,95 \\
\hline \multicolumn{4}{|c|}{$D[4]$} \\
\hline 6,07 & 3,89 & 5,42 & 5,21 \\
\hline 9,96 & 10,07 & 8,45 & 10,12 \\
\hline 5,89 & 9,98 & 7,06 & 3,90 \\
\hline \multicolumn{4}{|c|}{$D[5]$} \\
\hline 8,11 & 8,18 & 7,99 & 8,39 \\
\hline 10,07 & 4,98 & 9,99 & 5,02 \\
\hline 3,99 & 8,14 & 3,99 & 7,94 \\
\hline
\end{tabular}

Os valores de $\beta[i i \mid j j]$ calculados pela Eq. (4.10) podem expressar (em função dos protótipos) a consistência (ou similaridade) entre os sítios de dados. Tendo isso em mente, foi realizada uma análise comparativa, empregando-se o conjunto de dados Sin1 nos seguintes experimentos:

- Experimento I: foi usado o algoritmo CFC com nível de interação $\beta=0,25$ (informado);

- Experimento II: foi usado o algoritmo CFC- $\beta_{d}$ com nível de interação $\beta[i i \mid j j]$ (estimado);

Nesses dois experimentos o processo de colaboração foi monitorado usando-se a Eq. (4.10). No Experimento I esse monitoramento é denotado por $\beta^{M}[i i \mid j j]-M$ representa que a Eq. (4.10) foi usada apenas para monitorar o processo de colaboração do algoritmo CFC com nível de interação $\beta=0,25$ (informado pelo usuário). No Experimento II, por sua vez, este monitoramento é denotado por $\beta^{E}[i i \mid j j]-E$ representa que a Eq. (4.10), além de monitorar o processo de colaboração, foi também incorporada no algoritmo sendo usada para estimar os níveis de interação entre os pares de sítios em cada estágio de colaboração. As Figuras 5.7 - 5.11 ilustram o comportamento de $\beta^{M}[i i \mid j j]$ no Experimento I e $\beta^{E}[i i \mid j j]$ no Experimento II. Em particular, cada gráfico refere-se ao $i i$-ésimo sítio de dados em relação aos $j j$-ésimos sítios, $j j \neq i i$. Por exemplo, a Figura 5.7 ilustra as curvas de $\beta^{M}[i i \mid j j]$ e de $\beta^{E}[i i \mid j j]$ referentes ao sítio de dados $D[1]$ — em função dos sítios $D[2], D[3], D[4]$ e $D[5]$ durante 20 estágios de colaboração. 


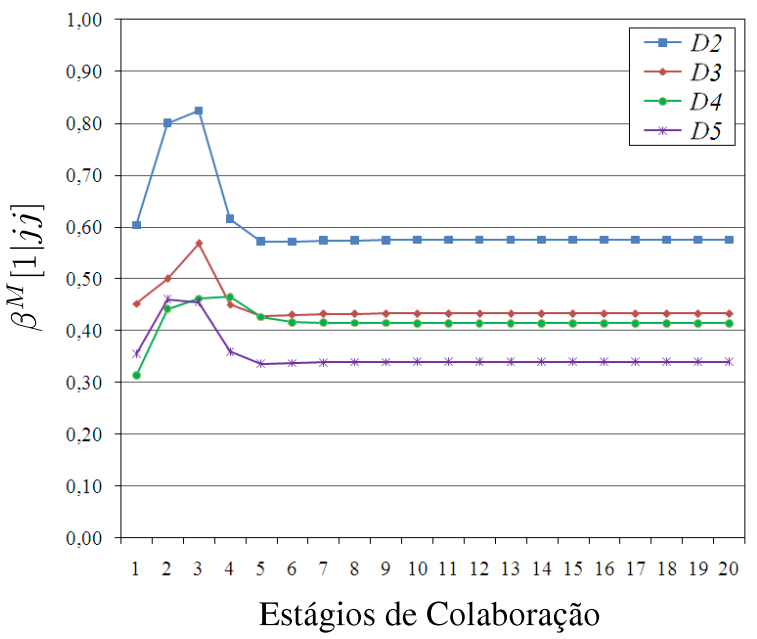

Experimento I

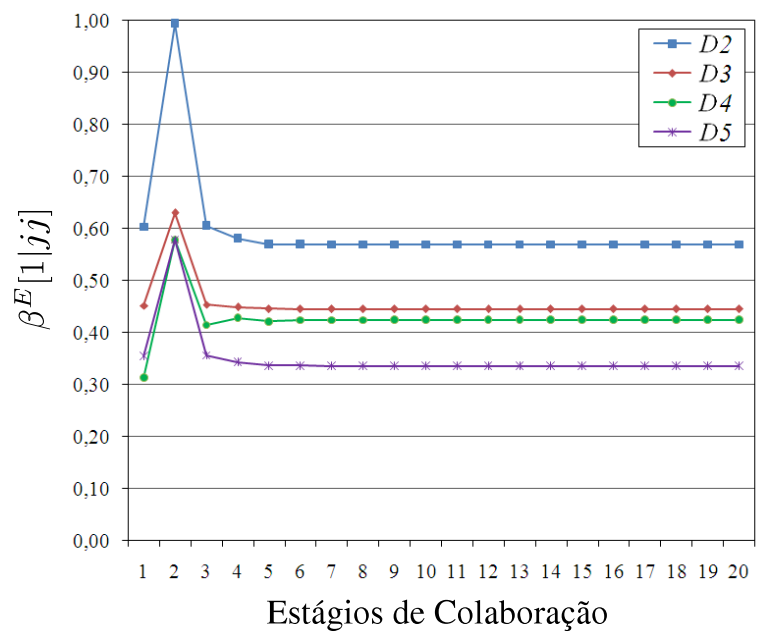

Experimento II

Figura 5.7: Monitoramento do processo de colaboração no sítio $D[1]$.

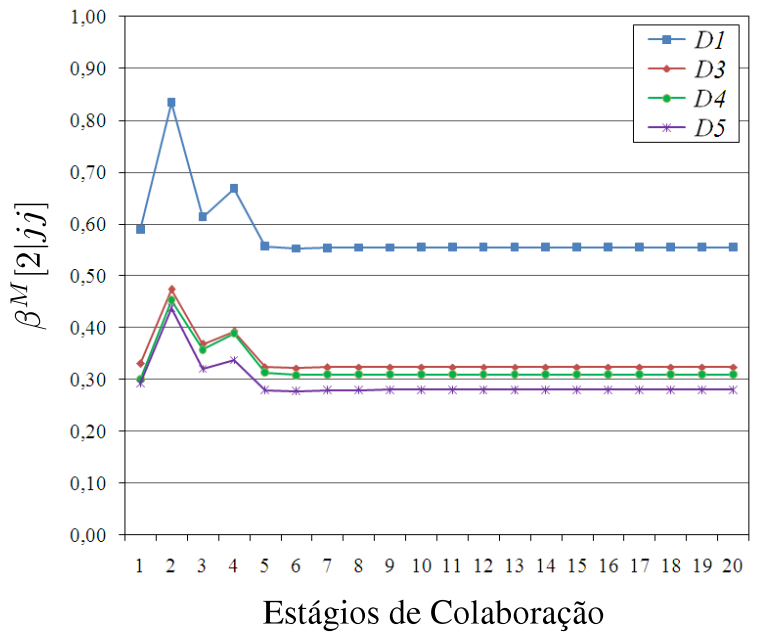

Experimento I

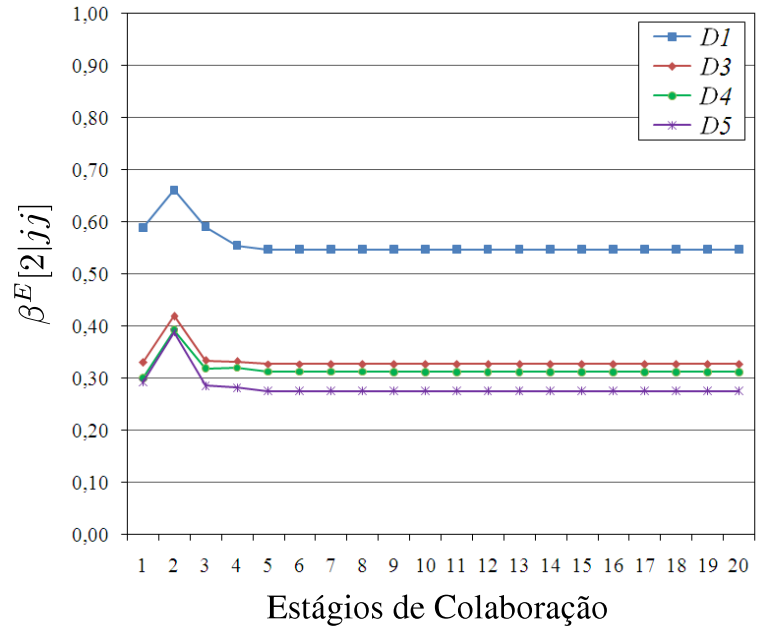

Experimento II

Figura 5.8: Monitoramento do processo de colaboração no sítio $D[2]$.

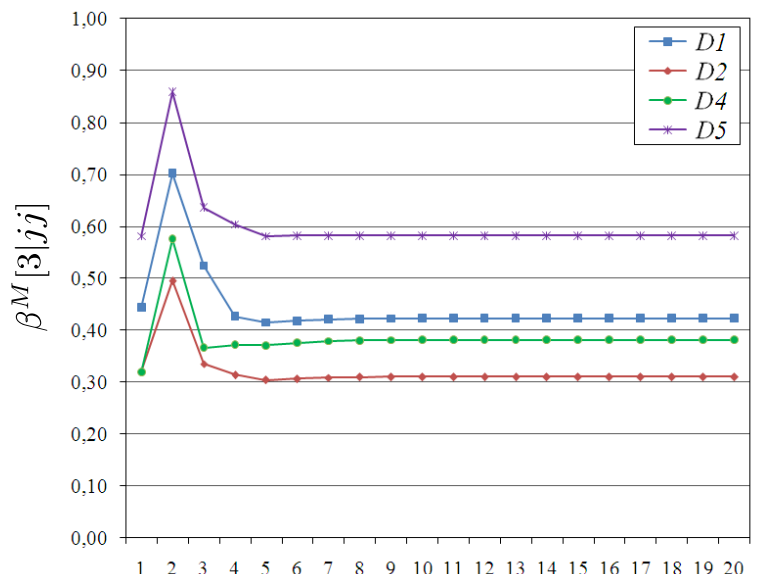

Estágios de Colaboração

Experimento I

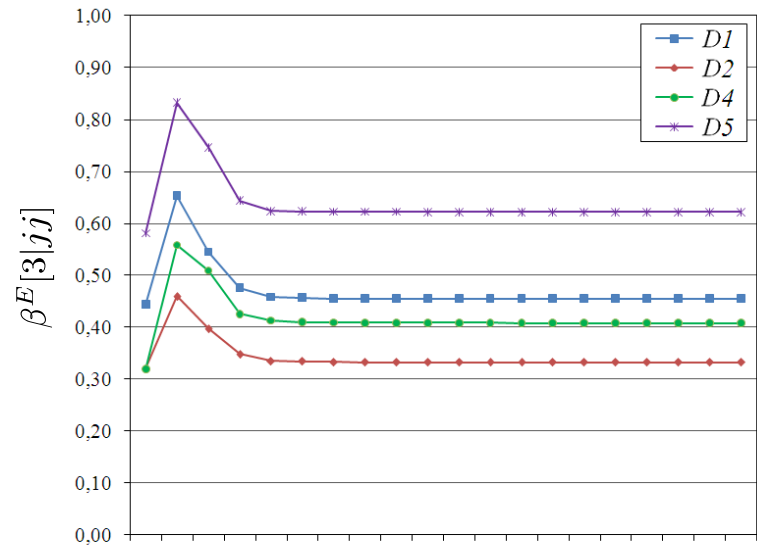

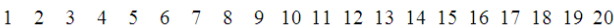
Estágios de Colaboração

Experimento II

Figura 5.9: Monitoramento do processo de colaboração no sítio $D[3]$. 

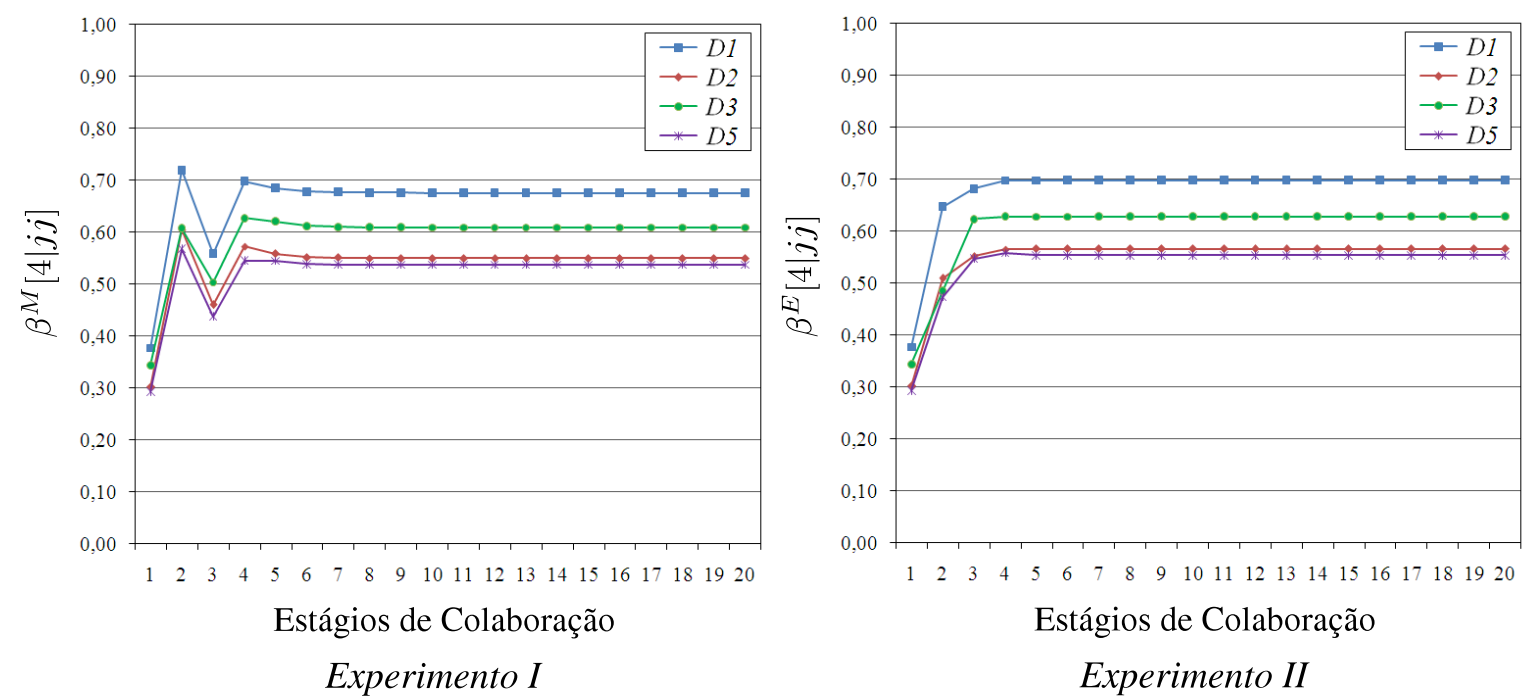

Figura 5.10: Monitoramento do processo de colaboração no sítio $D[4]$.
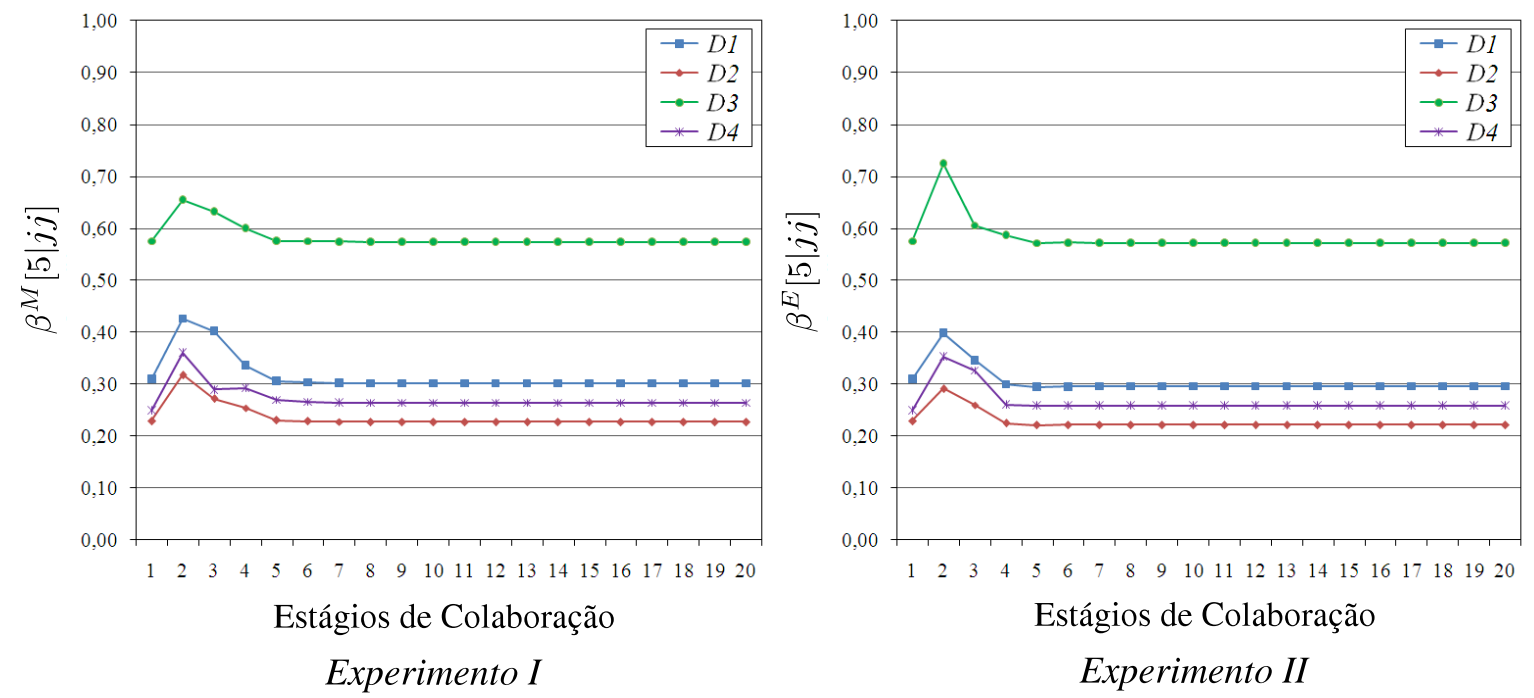

Figura 5.11: Monitoramento do processo de colaboração no sítio $D[5]$.

As Figuras $5.7-5.11$ mostram que os valores de $\beta^{M}[i i \mid j j]$ e $\beta^{E}[i i \mid j j], i i, j j=1, \ldots, P$, convergem para valores muito parecidos. Esse fato reforça a suposição de que o algoritmo CFC- $\beta_{d}$ manteve as características de seu algoritmo predecessor (CFC), porém com a vantagem de não ser mais necessária a informação do parâmetro $\beta$. Além disso, ao observar com maior atenção as figuras, pode-se notar que os valores finais de $\beta[i i \mid j j]$ convergem para valores muito semelhantes aos valores iniciais. A exceção fica por conta do sítio $D[4]$ (ver Figura 5.10), para o qual os valores iniciais e finais foram significativamente diferentes, sugerindo que este sítio possui estrutura de grupos bem diferente de todos os outros. Por tal razão, em $D[4]$ ocorreram mudanças mais significativas nos protótipos em relação as mudanças ocorridas nos protótipos dos outros sítios (conforme mostra a Tabela 5.2).

Os resultados observados no Experimento I e no Experimento II motivaram a concepção do algoritmo CFC- $\beta_{f}$ (Seção 4.3.1). Esse algoritmo estima, assim como o algoritmo CFC- $\beta_{d}$, os níveis de interação para cada par de sítios. Entretanto, tais valores são mantidos fixos du- 
rante a Fase Colaborativa procurando assegurar, assim, uma maior conformidade com os dados (que não mudam no decorrer do processo de colaboração). Esse algoritmo foi empiricamente avaliado nos conjuntos de dados $\operatorname{Sin} 1$ e Sin2. A Tabela 5.3 indica as coordenadas $(x, y)$ dos protótipos antes e depois do processo de colaboração no conjunto Sin1. Nota-se que, mantendo-se fixo $\beta[i i \mid j j]$, obteve-se protótipos muito similares aos protótipos obtidos pelos algoritmos CFC e CFC- $\beta_{d}$ - compare essa tabela com as Tabelas 5.1 e 5.2. A Tabela 5.4 apresenta os valores de $\beta[i i \mid j j]$, $i i, j j=1, \ldots, P$, estimados (e mantidos fixos) pelo algoritmo CFC- $\beta_{f}$. Por esta tabela nota-se que os níveis de interação entre os sítios de dados não são simétricos, i.e., $\beta[i i \mid j j] \neq \beta[j j \mid i i], i i \neq j j$. Além disso, esses valores reforçam a suposição de que o sítio $D[4]$ é relativamente menos semelhante comparativamente aos demais, apresentando valores relativamente menores para $\beta[4 \mid j j], j j=\{1,2,3,5\}$, para a maioria dos sítios (ver quinta coluna da Tabela 5.4). Por fim, a Figura 5.12 apresenta o comportamento de $W$ em 20 estágios de colaboração. Por este gráfico nota-se que o algoritmo CFC- $\beta_{f}$ obteve $W_{F}=2,02$ (valor de $W$ minimizado após o processo de colaboração). Este valor está dentro do intervalo formado pelos valores obtidos pelos algoritmos CFC e CFC- $\beta_{d}$ (que foram, respectivamente, 2,04 e 1,91). Portanto, para o conjunto Sin1, o algoritmo CFC- $\beta_{f}$ apresentou um desempenho muito similar aos seus predecessores.

Tabela 5.3: Protótipos obtidos com o algoritmo CFC- $\beta_{f}-$ Conjunto Sin1.

\begin{tabular}{|c|c|c|c|}
\hline \multicolumn{3}{|c|}{ Após Fase Inicial } & Após Fase Colaborativa \\
\hline \multicolumn{4}{|c|}{$D[1]$} \\
\hline 8,53 & 10,07 & 8,53 & 10,04 \\
\hline 3,94 & 4,40 & 3,93 & 4,51 \\
\hline 10,00 & 4,02 & 9,94 & 4,00 \\
\hline \multicolumn{4}{|c|}{$D[2]$} \\
\hline 4,52 & 4,46 & 4,45 & 4,56 \\
\hline 10,51 & 3,82 & 10,42 & 3,87 \\
\hline 10,54 & 10,12 & 10,47 & 10,15 \\
\hline \multicolumn{4}{|c|}{$D[3]$} \\
\hline 10,09 & 5,87 & 10,02 & 5,52 \\
\hline 4,35 & 5,94 & 4,44 & 6,12 \\
\hline 7,09 & 9,05 & 7,61 & 8,95 \\
\hline \multicolumn{4}{|c|}{$D[4]$} \\
\hline 6,07 & 3,89 & 5,40 & 5,53 \\
\hline 9,96 & 10,07 & 8,49 & 10,14 \\
\hline 5,89 & 9,98 & 6,84 & 3,83 \\
\hline \multicolumn{4}{|c|}{$D[5]$} \\
\hline 8,11 & 8,18 & 8,02 & 8,38 \\
\hline 10,07 & 4,98 & 9,99 & 5,01 \\
\hline 3,99 & 8,14 & 4,00 & 7,96 \\
\hline
\end{tabular}


Tabela 5.4: Valores de $\beta[i i \mid j j]$ para todos os pares de sítios — Conjunto Sin1.

\begin{tabular}{|c|c|c|c|c|c|}
\hline & $D[1]$ & $D[2]$ & $D[3]$ & $D[4]$ & $D[5]$ \\
\hline$D[1]$ & 0,00 & 0,60 & 0,45 & 0,31 & 0,35 \\
\hline$D[2]$ & 0,59 & 0,00 & 0,33 & 0,30 & 0,29 \\
\hline$D[3]$ & 0,44 & 0,32 & 0,00 & 0,32 & 0,58 \\
\hline$D[4]$ & 0,38 & 0,30 & 0,34 & 0,00 & 0,29 \\
\hline$D[5]$ & 0,31 & 0,23 & 0,57 & 0,25 & 0,00 \\
\hline
\end{tabular}

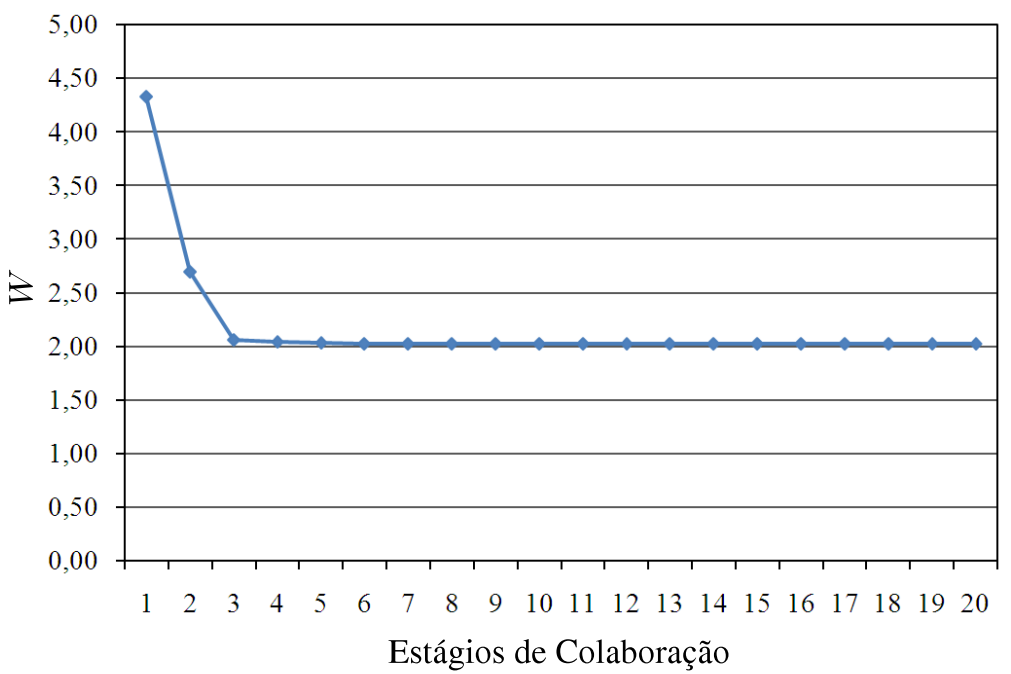

Figura 5.12: Comportamento de $W-$ Algoritmo CFC- $\beta_{f}-$ Conjunto Sin1.

A Figura 5.13 ilustra o comportamento de $W$ para o algoritmo CFC- $\beta_{f}$ no conjunto $\operatorname{Sin} 2$ $(c=2)$. Os níveis de interação $\beta[1 \mid 2]=0,20$ e $\beta[2 \mid 1]=0,22$ foram estimados (e mantidos fixos) durante o processo de colaboração. Após 20 estágios de colaboração o algoritmo obteve $W_{F}=0,42$. Esse valor foi aproximadamente o dobro do que aquele obtido pelo algoritmo CFC. No entanto, o algoritmo CFC foi executado $\operatorname{com} \beta=0,95$, o que implica em uma forte colaboração entre os sítios de dados e, consequentemente, a obtenção de valores muito baixos de $W$. Novamente é importante enfatizar que nem sempre uma forte colaboração entre os sítios de dados é desejável - especialmente ao assumir que os dados distribuídos entre os sítios são provenientes de diferentes populações.

A Figura 5.14 ilustra os grupos obtidos pelo algoritmo CFC- $\beta_{f}$. Nessas partições, $p i$ indica a posição inicial dos protótipos após a Fase Inicial e $p f$ a posição dos protótipos depois da Fase Colaborativa. Nota-se que os protótipos não se alteraram de forma significativa depois do processo de colaboração. No entanto, os protótipos se reposicionaram na direção esperada, sendo similares aos protótipos obtidos pelo algoritmo CFC com $\beta=0,95$ (ver Figura 5.5). Nesse sentido, o algoritmo CFC- $\beta_{f}$ apresentou um comportamento similar ao algoritmo CFC, com a vantagem de não ser necessária a informação do nível de interação $\beta$, o qual é estimado automaticamente, para cada par de sítios, e mantido fixo durante os estágios de colaboração - assim, o CFC- $\beta_{f}$ é também mais eficiente que o CFC- $\beta_{d}$ (que estima esses valores a cada estágio de colaboração). Finalmente, convém enfatizar que os três algoritmos abordados nessa seção (CFC, CFC- $\beta_{d}$ e CFC- $\beta_{f}$ ) necessitam da informação do número de grupos (c), o qual é fixo para todos os sítios de dados durante todos os estágios de colaboração. O experimento 
descrito a seguir ilustra a importância de se estimar o número correto de grupos.

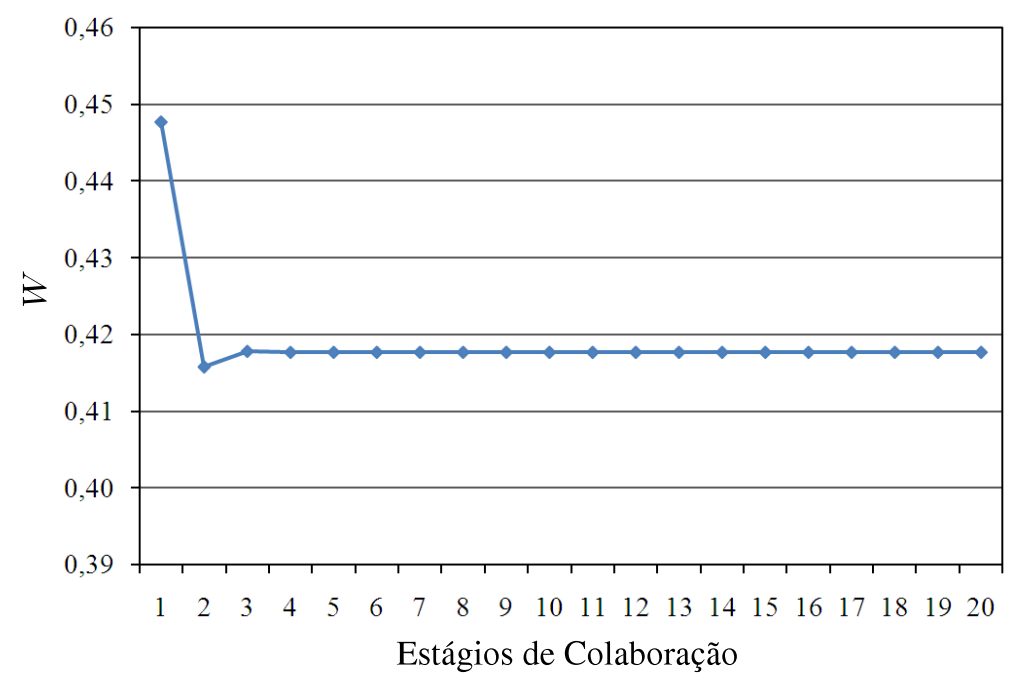

Figura 5.13: Comportamento de $W$ - Algoritmo CFC- $\beta_{f}-$ Conjunto Sin2.
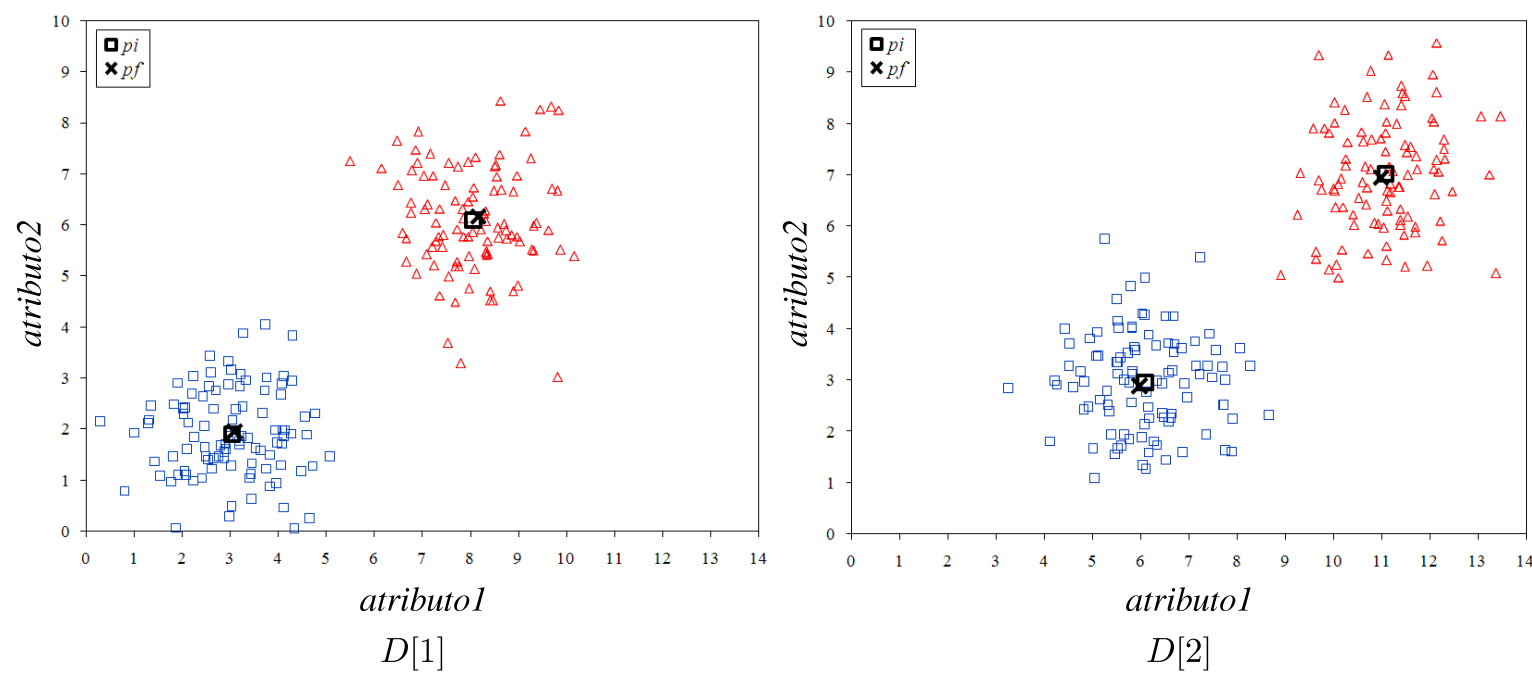

Figura 5.14: Grupos obtidos com o algoritmo CFC- $\beta_{f}-$ Conjunto Sin2.

\subsubsection{Colaboração com Mudanças na Estrutura dos Dados — Algoritmos CFC, CFC- $\beta_{d}$ e CFC- $\beta_{f}$}

O experimento reportado nessa seção teve como objetivo analisar o comportamento dos algoritmos CFC, CFC- $\beta_{d}$ e CFC- $\beta_{f}$ diante de mudanças ocorridas na estrutura de dados de um dos sítios envolvidos na colaboração. Os impactos provenientes dessas mudanças foram avaliados pela movimentação dos protótipos durante o processo de colaboração. O conjunto de dados usado, $\operatorname{Sin} 3$, consiste dos sítios $D[1]$ e $D[2]$ ilustrados na Figura 5.15. No decorrer do experimento a estrutura do sítio $D[2]$ passou a ser dinamicamente incrementada com novos objetos, tornando-se mais parecida com a estrutura do sítio $D[1]$. Essas mudanças nos dados podem ocorrer em situações reais como, por exemplo, ao longo de vários processos de colaboração 

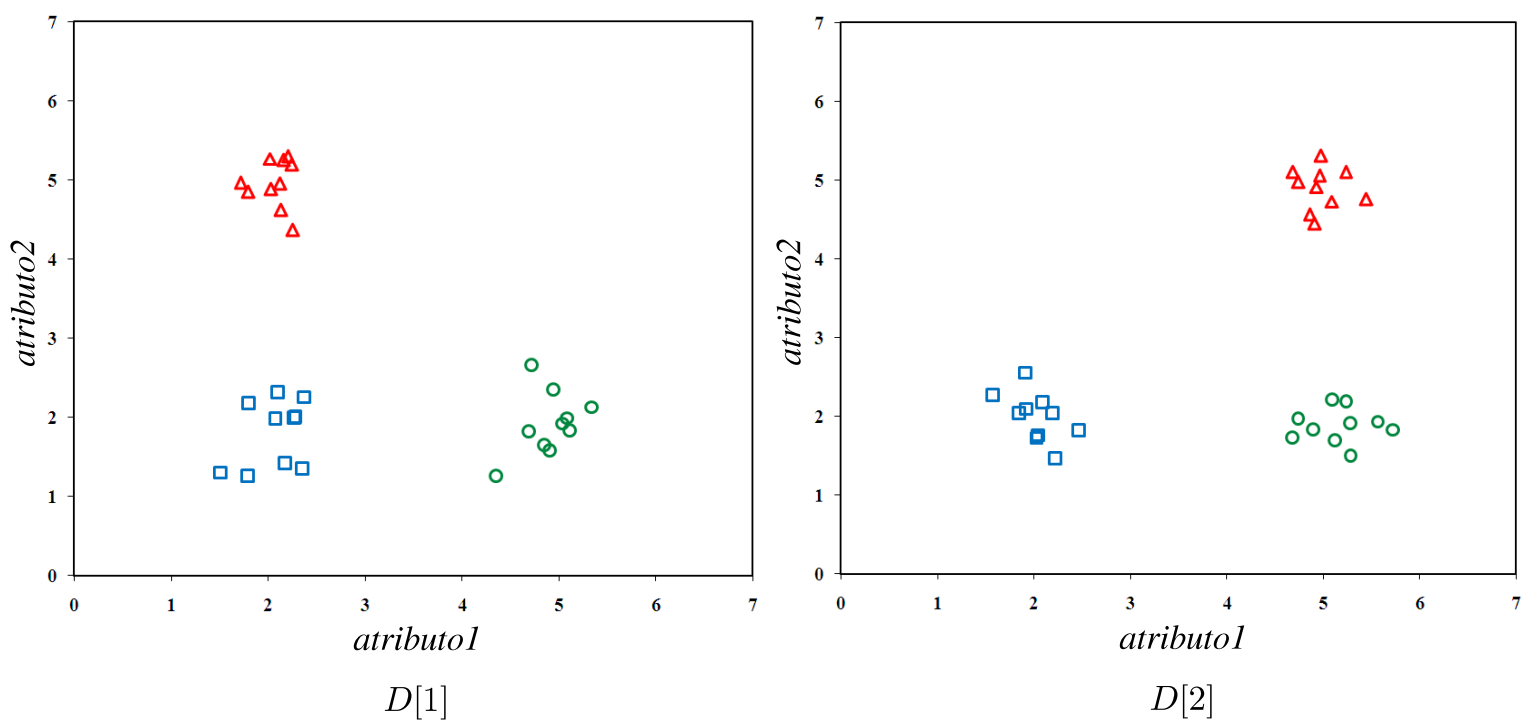

Figura 5.15: Sítios de dados do conjunto $\operatorname{Sin} 3$.

entre duas agências de uma instituição financeira. Neste caso, em especial, pode-se dizer que, enquanto a primeira agência (sítio $D[1]$ ) manteve em um determinado período o mesmo número de clientes, a segunda agência (sítio $D[2]$ ), nesse mesmo período, passou a contar com novos clientes (com características semelhantes àquelas apresentadas por clientes da primeira agência - e.g., compras com cartão e renda). Nesse sentido, a partir do sítio $D[2]$ pode-se derivar os sítios $D[2]_{0}, D[2]_{1}, \ldots, D[2]_{5}$, nos quais cada sub-índice indica o número de novos objetos (clientes) incrementados no decorrer do tempo. Por exemplo, $D[2]_{0}$ não teve nenhum objeto incrementado, correspondendo, assim, ao próprio sítio $D[2]$. $D[2]_{1}$, por sua vez, teve um objeto incrementado (de acordo com distribuição Gaussiana usada para gerar $D[1]$, descrita pelo vetor médio $[2,0 ; 5,0]$ e variância para cada coordenada igual a 0,3$)$. Os outros sítios $\left(D[2]_{2}, \ldots, D[2]_{5}\right)$ foram obtidos de forma análoga ao sítio $D[2]_{1}$, i.e., incrementando-se um novo objeto ao sítio antecessor (e.g., o sítio $D[2]_{2}$ foi gerado a partir do incremento de mais um objeto ao sítio $\left.D[2]_{1}\right)$. O algoritmo CFC- $\beta_{f}$ foi executado em 6 diferentes instantes de tempo. Para cada instante de tempo foi considerado um par de sítios, ou seja, o sítio $D[1]$ e um outro sítio dentre aqueles gerados a partir de $D[2]\left(D[2]_{0}, D[2]_{1}, \ldots, D[2]_{5}\right)$. De maneira mais específica, para o Instante 1 usou-se os sítios $D[1]$ e $D[2]_{0}$. Para o Instante 2 usou-se os sítios $D[1]$ e $D[2]_{1}$ e, assim, sucessivamente até o Instante 6, no qual foram usados os sítios $D[1]$ e $D[2]_{5}$. Os resultados do comportamento do algoritmo CFC- $\beta_{f}(\operatorname{com} c=3)$ nos diferentes instantes de tempo podem ser sumarizados pelas Figuras 5.16 - 5.18. Essas figuras ilustram os grupos dos sítios de dados indicando os protótipos em dois momentos distintos: pi refere-se aos protótipos iniciais, obtidos após a Fase Inicial do algoritmo (sem colaboração), e $p f$ refere-se aos protótipos finais, obtidos após a Fase Colaborativa (20 estágios de colaboração). Dado que não foram observadas mudanças significativas nos protótipos do sítio $D[1]$ em nenhum dos instantes de tempo, podese concluir que este sítio não se beneficiou do processo de colaboração (ver Figura 5.16). Por outro lado, os sítios de dados $D[2]_{0}, D[2]_{1}, \ldots, D[2]_{5}$ apresentaram diferentes resultados. Para o sítio de dados $D[2]_{0}$ (Instante 1), observa-se na Figura 5.17 que não houve mudanças significativas entre os protótipos iniciais ( $p i)$ e protótipos finais $(p f)$. Contudo, quanto mais objetos 
foram incrementados, a cada instante de tempo, revelando uma nova estrutura em $D[2]$ - mais parecida com a do sítio $D[1]$ —, maior foi a diferença entre os protótipos iniciais e finais. Esse fato pode ser constatado pela Figura 5.18 (sítio $D[2]_{5}$ — Instante 6), na qual o protótipo posicionado inicialmente nas coordenadas $(4,7 ; 4,9)$ após o processo de colaboração se posicionou em $(3,6 ; 4,9)$, ficando mais próximo dos cinco objetos incrementados. É importante ressaltar que resultados muito similares a esses foram encontrados ao se utilizar os algoritmos CFC e CFC- $\beta_{d}$. Portanto, o processo de colaboração realizado por esses algoritmos buscou manter um certo grau de consistência entre os sítios de dados. No entanto, esse experimento também ilustra problemas que podem surgir quando o número de grupos é pré-determinado a priori. Em particular, a Figura 5.18 sugere fortemente a presença de 4 grupos, enquanto que os algoritmos foram executados assumindo-se a existência de apenas 3 grupos.

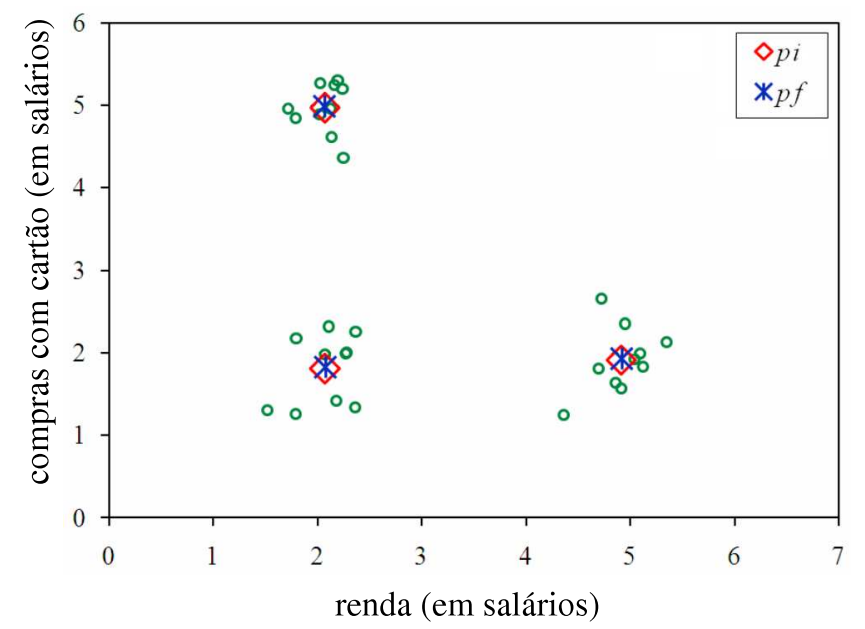

Figura 5.16: As posições dos protótipos iniciais e finais para o sítio $D[1]$ em todos os instantes de tempo permaneceram muito próximas das posições ilustradas nesta figura.

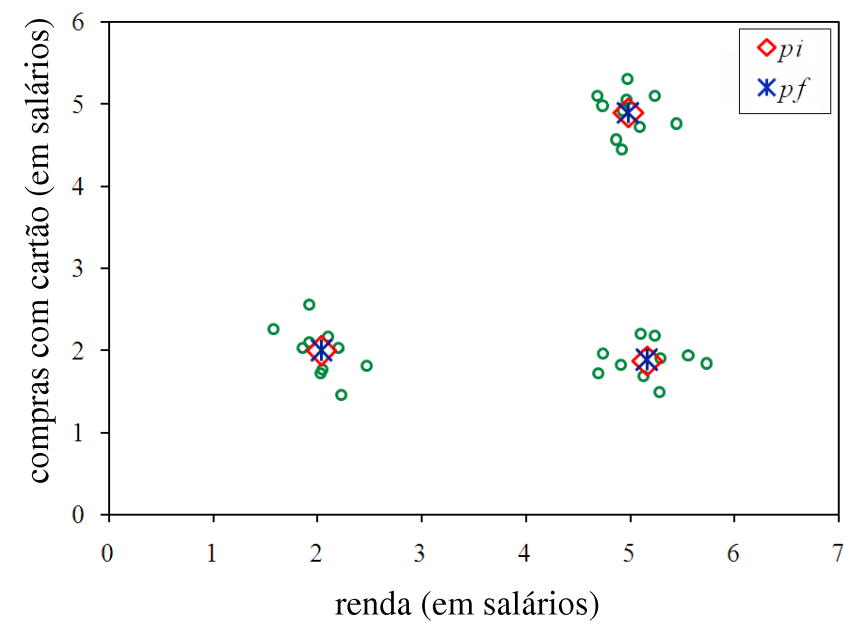

Figura 5.17: Grupos do sítio de dados $D[2]_{0}$ — Instante 1. 


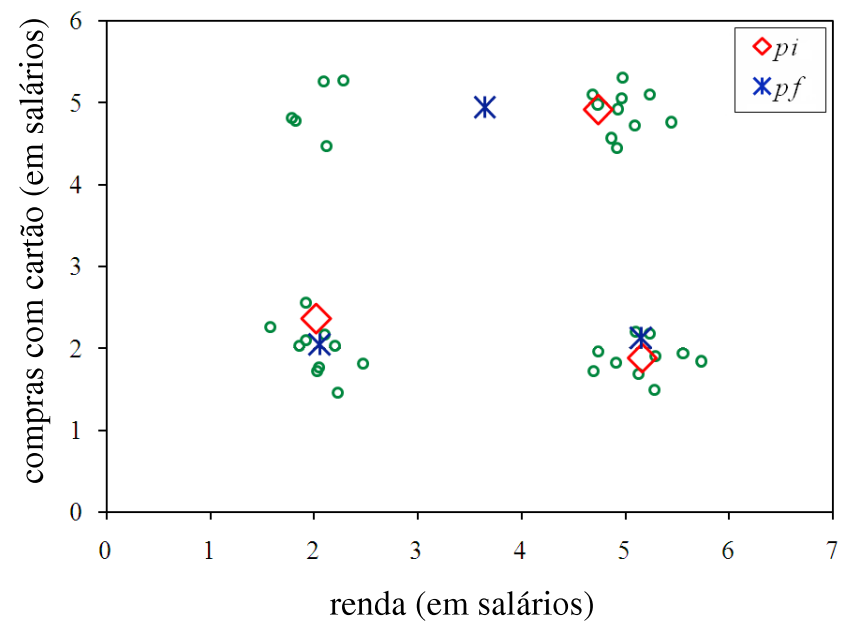

Figura 5.18: Grupos do sítio de dados $D[2]_{5}$ — Instante 6.

\subsection{Exemplos Ilustrativos - Cenários de Aplicação}

Embora existam várias medidas para se avaliar resultados de agrupamento, incluindo inúmeros critérios de validade - ver, por exemplo, (Vendramin et al., 2010) —, a avaliação desses resultados é, em geral, uma tarefa complicada. Esse fato é particularmente válido ao se considerar algoritmos para agrupamento fuzzy colaborativo. Na realidade, não existem ainda metodologias consolidadas para se avaliar algoritmos dessa natureza. De maneira mais específica, não há ainda um consenso fundamentado sobre qual deveria ser o resultado esperado da aplicação de um algoritmo de agrupamento fuzzy colaborativo em um determinado número de sítios de dados (em especial, quando os dados são provenientes de diferentes populações). Nesse sentido, essa seção apresenta alguns exemplos ilustrativos que destacam as diferenças de desempenho entre os algoritmos estudados levando-se em conta suposições sobre os dados (ver Seção 4.4). Embora a análise realizada é inerentemente subjetiva (assim como é a definição do que é um grupo), ela pode ser útil ao usuário interessado em aplicar algoritmos de agrupamento fuzzy colaborativo na prática. Tal análise se baseia nos cenários de aplicação ilustrados pela Figura 4.3. Em particular, o Cenário 1 compreende os algoritmos apropriados quando os dados que estão distribuídos nos diferentes sítios são provenientes de diferentes populações. Por outro lado, o Cenário 2 envolve os algoritmos adequados quando os dados distribuídos são de uma mesma população. As Seções 5.3.1 e 5.3.2 abordam, respectivamente, esses cenários. Além disso, a Seção 5.3.3 aborda experimentos que ilustram o comportamento dos algoritmos desenvolvidos na presença de dados aleatórios. Por fim, a Seção 5.3.4 envolve uma análise experimental em conjuntos de dados multivariados.

\subsubsection{Experimentos com Dados Bi-Dimensionais Provenientes de Diferen- tes Populações}

O primeiro exemplo dessa seção envolve o conjunto de dados Sin4. Contudo, antes de aplicar qualquer algoritmo para agrupamento fuzzy colaborativo, buscou-se explorar a estrutura de grupos presente em cada um dos sítios de dados desse conjunto. Nesse sentido, aplicando- 
se, em cada um dos sítios de dados (individualmente), o algoritmo FCM- $c^{*}$ (Algoritmo 3 Seção 2.4.4) $\operatorname{com} c_{\max }=6$ e $n_{p}=100$, juntamente com a Silhueta Fuzzy Simplificada (SFS), obteve-se (como esperado) as partições de dados ilustradas na Figura 5.19. Da mesma forma, aplicando-se o algoritmo FCM-c* no conjunto de dados formado por $D[1] \cup D[2]$ (i.e., os dados dos dois sítios foram centralizados em um único repositório) obteve-se como resultado a partição ilustrada na Figura 5.20. É importante salientar que esse último resultado também é esperado para os algoritmos que assumem que os dados são provenientes de uma mesma população (Cenário 2 - Seção 5.3.2), o que não se constitui no presente cenário avaliado. Ao assumir que os dados são provenientes de diferentes populações (Cenário 1), os algoritmos sugeridos de acordo com a Figura 4.3 são: CFC, CFC- $\beta_{d}$, CFC- $\beta_{f}$ e CFCM- $\beta_{f}-c^{*}$. Desses algoritmos, somente o último é capaz de automaticamente estimar o número de grupos. Para os demais, foi fornecido o número correto de grupos $(c=2)$.
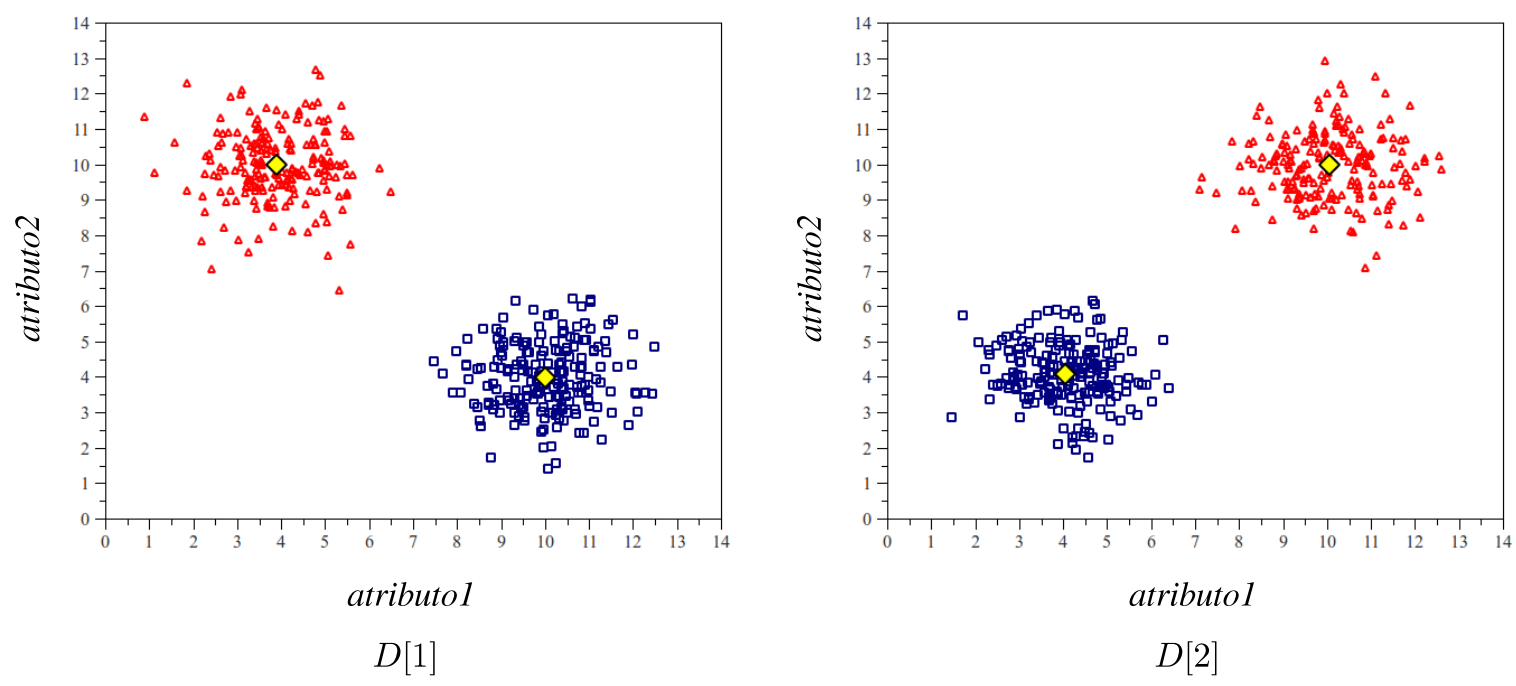

Figura 5.19: Grupos obtidos em cada sítio pelo algoritmo FCM-c* - Conjunto Sin4.

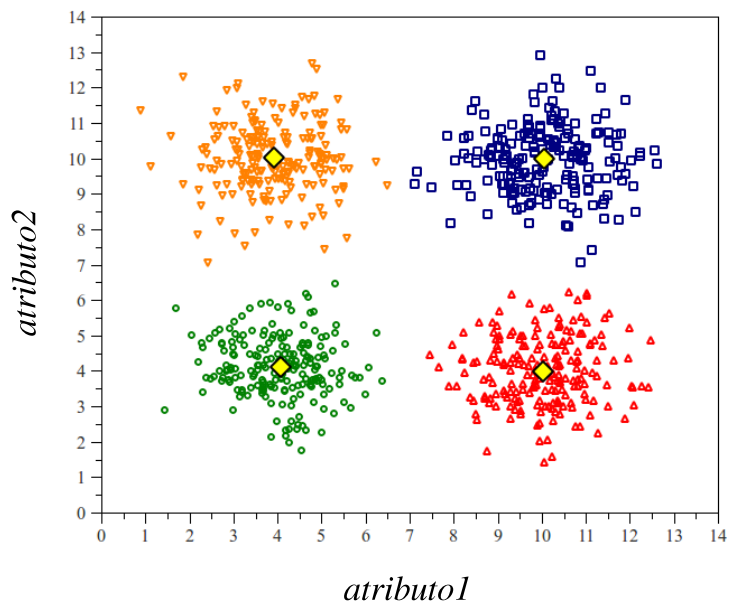

Figura 5.20: Grupos obtidos pelo algoritmo FCM- $c^{*}$ — Conjunto $\operatorname{Sin} 4(D[1] \cup D[2])$.

A Figura 5.21 ilustra os resultados obtidos pelo algoritmo CFC- $\beta_{f}$ após quatro estágios de colaboração. Nota-se que os grupos obtidos são praticamente idênticos àqueles ilustrados na Figura 5.19, exceto por pequenas diferenças com relação à posição dos protótipos. Essa ob- 
servação sugere que não houve atividade colaborativa significante (de maneira que o sítio $D[1]$ não foi afetado pelo agrupamento muito diferente presente no sítio $D[2]$, e vice-versa). Esse fato é desejado, neste caso, ao assumir que os dados são de diferentes populações. Resultados muito similares a este foram também alcançados usando-se os algoritmos $\mathrm{CFC}(\operatorname{com} \beta=0,10$, otimizado a partir dos dados executando-se o algoritmo para diferentes valores de $\beta$ ) e CFC$\beta_{d}$. Todos esses algoritmos (CFC, CFC- $\beta_{d}$ e $\mathrm{CFC}-\beta_{f}$ ) requerem que o número de grupos seja fornecido pelo usuário. Quando o número de grupos não é conhecido pode-se usar o algoritmo CFCM- $\beta_{f}-c^{*}$, o qual estima tal valor automaticamente a partir dos dados. Esse algoritmo foi também aplicado no conjunto de dados $\operatorname{Sin} 4$, usando-se $c_{\max }=6, n_{p}=100$, e a SFS como índice de validade. Os protótipos obtidos após dois estágios de colaboração foram muito semelhantes àqueles ilustrados na Figura 5.19. Esse algoritmo também estimou automaticamente (a partir dos dados, antes do início do processo de colaboração) os níveis de interação entre os sítios. É importante lembrar que esses níveis de interação são assimétricos — ver Eq. (4.10). Para evitar essa potencial dificuldade, foi usada a média entre cada par de sítios. Assim, para o conjunto de dados $\operatorname{Sin} 4$, em particular, foi estimado $\beta[1 \mid 2]=\beta[2 \mid 1]=(\beta[1 \mid 2]+\beta[2 \mid 1]) / 2=0,09$. Esse baixo nível de interação é esperado para esse conjunto de dados, o que indica a grande diferença existente entre as estruturas de grupos dos sitios $D[1]$ e $D[2]$. Como resultado, os grupos encontrados pelo algoritmo CFCM- $\beta_{f}-c^{*}$ foram os mesmos ilustrados na Figura 5.19, o que é esperado ao assumir que os dados são de diferentes populações. Particularmente, neste caso, o algoritmo esteve em conformidade com a idéia de que grupos de populações muito diferentes não deveriam ser representados em cada um dos sítios de dados.

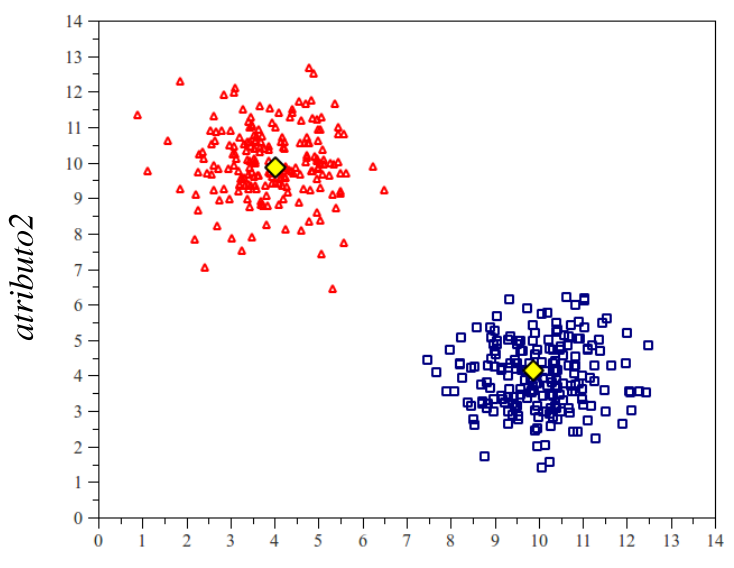

atributol

$D[1]$

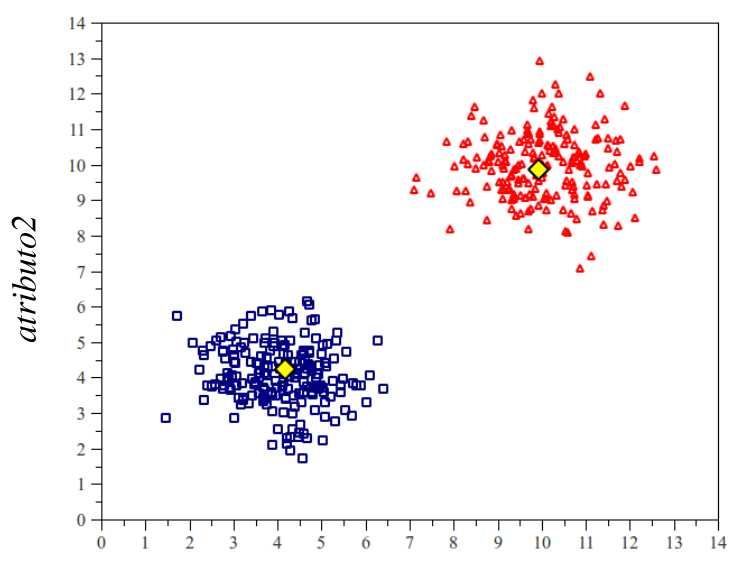

atributol

$D[2]$

Figura 5.21: Grupos obtidos pelo algoritmo CFC- $\beta_{f}$ - Conjunto Sin4.

Análise análoga também foi feita usando-se o conjunto de dados Sin5. Antes de aplicar os algoritmos para agrupamento fuzzy colaborativo, primeiramente foi aplicado o algoritmo FCM- $c^{*}\left(c_{\max }=6\right.$ e $\left.n_{p}=100\right)$ em cada sítio de dados $(D[1]$ e $D[2])$. A Figura 5.22 ilustra os grupos obtidos. Note que esses sítios de dados contém um certo número de objetos em regiões comuns do espaço bi-dimensional. Mais precisamente, esse sítios foram gerados a partir da seleção de alguns objetos do conjunto de dados da Figura 5.23, que representa, de fato, a união 
dos objetos dos sítios $D[1]$ e $D[2]$. A partir desse ponto de vista, é razoável afirmar que, de certa forma, esses sítios têm algum grau de similaridade entre si. A Figura 5.23 também ilustra os grupos que deveriam ser obtidos pelos algoritmos de agrupamento fuzzy colaborativo que assumem que os dados são provenientes de uma mesma população (Cenário 2). No entanto, considerando o Cenário 1 (que assume que os dados são de diferentes populações) a situação é diferente, i.e., os algoritmos de agrupamento fuzzy colaborativo deveriam ser capazes de identificar apenas estruturas de grupos em comum presentes nos sítios de dados. Isto é essencialmente o que se espera do processo de colaboração nesse cenário. Em outras palavras, grupos que são (parcialmente) similares deveriam ser refinados pelo processo de colaboração, enquanto que grupos completamente diferentes não deveriam ser afetados. Tendo isso em mente, os resultados dos algoritmos no conjunto Sin5 são apresentados a seguir.
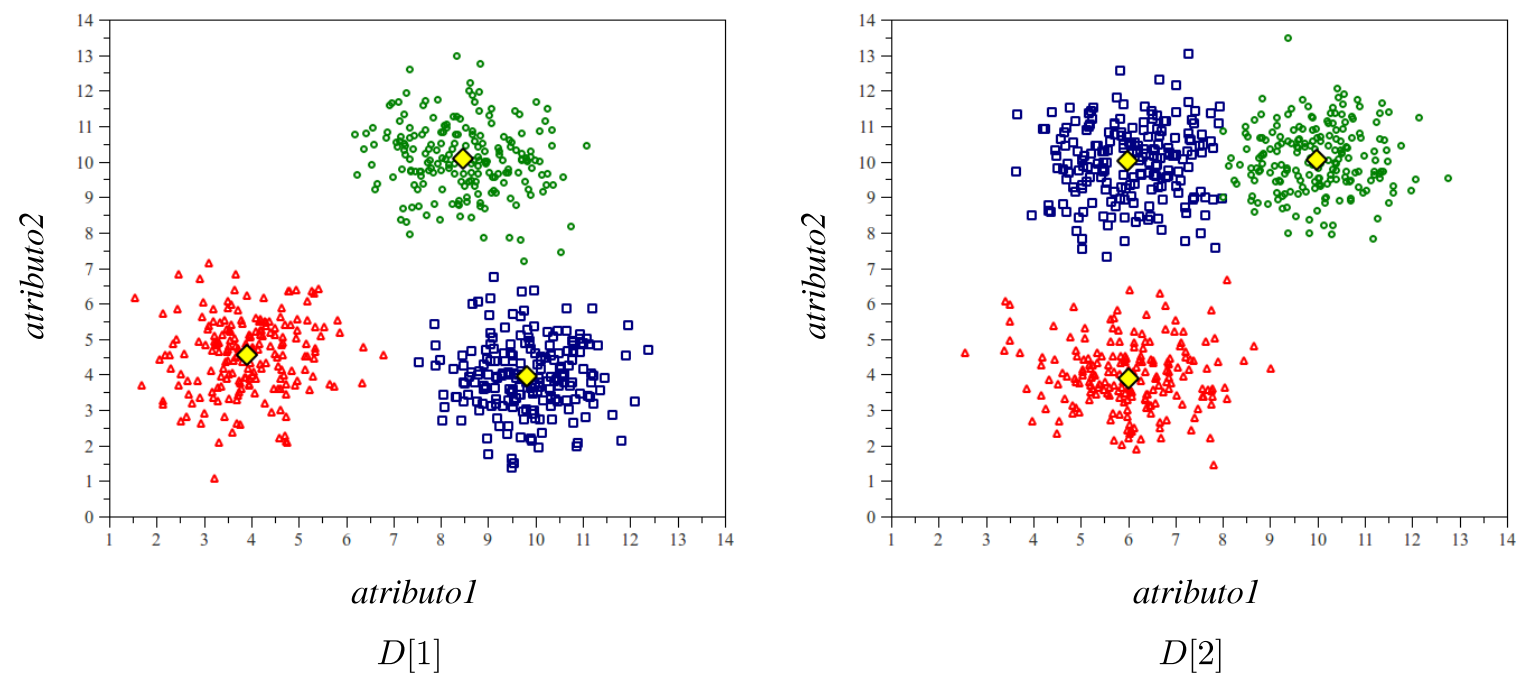

Figura 5.22: Grupos obtidos em cada sítio pelo algoritmo FCM-c* — Conjunto Sin5.

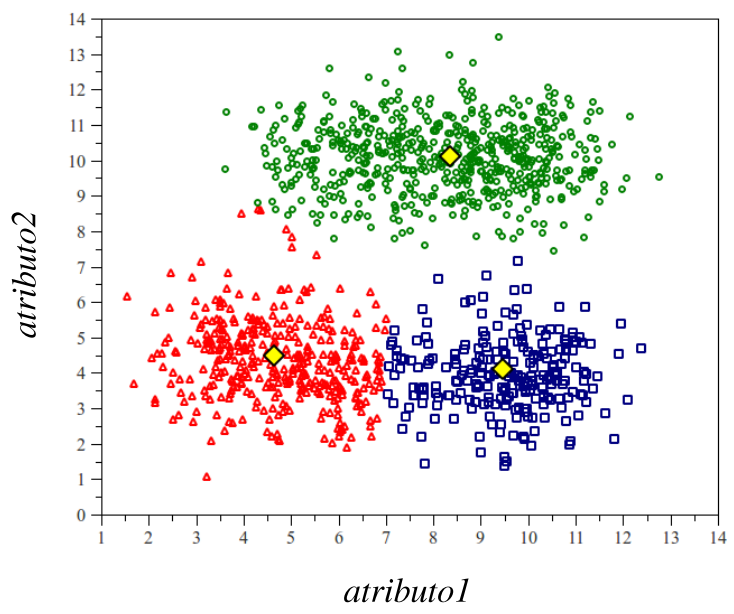

Figura 5.23: Grupos obtidos pelo algoritmo FCM- $c^{*}-$ Conjunto $\operatorname{Sin} 5(D[1] \cup D[2])$.

Os algoritmos CFC ( $\beta=0,35$, otimizado a partir dos dados) e CFC- $\beta_{d}$ (ambos $\operatorname{com} c=$ 3) não ocasionaram mudanças significativas nos protótipos de grupos após onze estágios de colaboração (i.e., os protótipos convergiram sem que houvesse ajustes significativos). A Figura 5.24 apresenta os grupos obtidos pelo algoritmo $\mathrm{CFC}$, os quais são muito similares àqueles 
ilustrados na Figura 5.22. Portanto, esse algoritmo fez apenas pequenos ajustes nos protótipos como resultado do processo de colaboração. Vale destacar que o algoritmo CFC- $\beta_{d}$ apresentou resultados muito parecidos. Por outro lado, o algoritmo $\mathrm{CFC}-\beta_{f}(\operatorname{com} c=3$ ) exibiu diferentes resultados, os quais são ilustrados na Figura 5.25. Essa figura indica que o grupo de objetos representados por triângulos em $D[1]$, com protótipo de coordenadas $(8,5 ; 10,1)$, influenciou o resultado no sítio de dados $D[2]$, de tal maneira que dois grupos desse sítio foram unidos durante o processo de colaboração - compare $D[2]$ da Figura $5.25 \mathrm{com} D[2]$ da Figura 5.22. Uma possível explicação para esse comportamento é que os objetos com atributo $2>7$ e atributol em torno de 8,5 em $D[2]$ poderiam ser considerados como sub-representados, por exemplo, devido a alguma particularidade do processo de amostragem. Por outro lado, objetos com características similares são encontrados em torno das coordenadas $(8,5 ; 10,1)$ em $D[1]$ (ver novamente Figura 5.25). O protótipo que representa esses objetos influenciou os resultados de agrupamento em $D[2]$, fazendo o algoritmo encontrar um único grupo formado por triângulos. Pode-se argumentar que, fazendo isso, o algoritmo CFC- $\beta_{f}$ está atuando como se os dados fossem de uma mesma população. Essa análise é correta para os grupos representados por triângulos na Figura 5.25. Contudo, explica apenas parte dos resultados encontrados por este algoritmo nesse sítio de dados, i.e., ainda é preciso considerar por quê o algoritmo dividiu o outro grupo de $D[2]$. Uma explicação plausível se baseia em duas observações: (i) o algoritmo é limitado a induzir um número fixo de grupos (três nesse caso); e (ii) os objetos representados por quadrados em $D[2]$ podem, de fato, serem considerados como pertencentes a um outro grupo (e.g., o grupo formado por quadrados na Figura 5.23). Note, entretanto, que em $D[2]$ da Figura 5.25, o protótipo do grupo formado por quadrados é muito diferente do respectivo protótipo na Figura 5.23, o qual deveria ser idealmente obtido por um algoritmo que (ao contrário do CFC- $\beta_{f}$ ) assume que os dados são de uma mesma população (como será abordado na próxima seção). Essa discussão pode ser melhor elaborada ao considerar os resultados obtidos pelo algoritmo CFCM- $\beta_{f}-c^{*}\left(c_{\max }=6, n_{p}=100\right)$. A Figura 5.26 ilustra os protótipos obtidos depois de quatro estágios de colaboração desse algoritmo $(\beta[1 \mid 2]=\beta[2 \mid 1]=0,35)$. Seguindo resultados semelhantes àqueles obtidos pelo algoritmo $\mathrm{CFC}-\beta_{f}$, os dois grupos superiores em $D[2]$ foram unidos - compare $D[2]$ da Figura $5.26 \mathrm{com} D[2]$ da Figura 5.22. Além disso, o grupo formado por círculos em $D[1]$ da Figura 5.26, representado pelo protótipo de coordenadas $(9,8 ; 3,9)$, não apresentou evidências suficientes (peso do protótipo) para ser representado em $D[2]$ da Figura 5.26. Esse resultado é interessante ao assumir dados de diferentes populações, de maneira que grupos muito diferentes, presentes em outros sítios, não deveriam exercer uma forte influência em um sítio de dados em particular. É importante notar também que o protótipo do grupo formado por triângulos em $D[2]$ da Figura 5.26 foi pouco ajustado pelo processo de colaboração, sendo muito similar ao protótipo do grupos formado por quadrados em $D[1]$. 

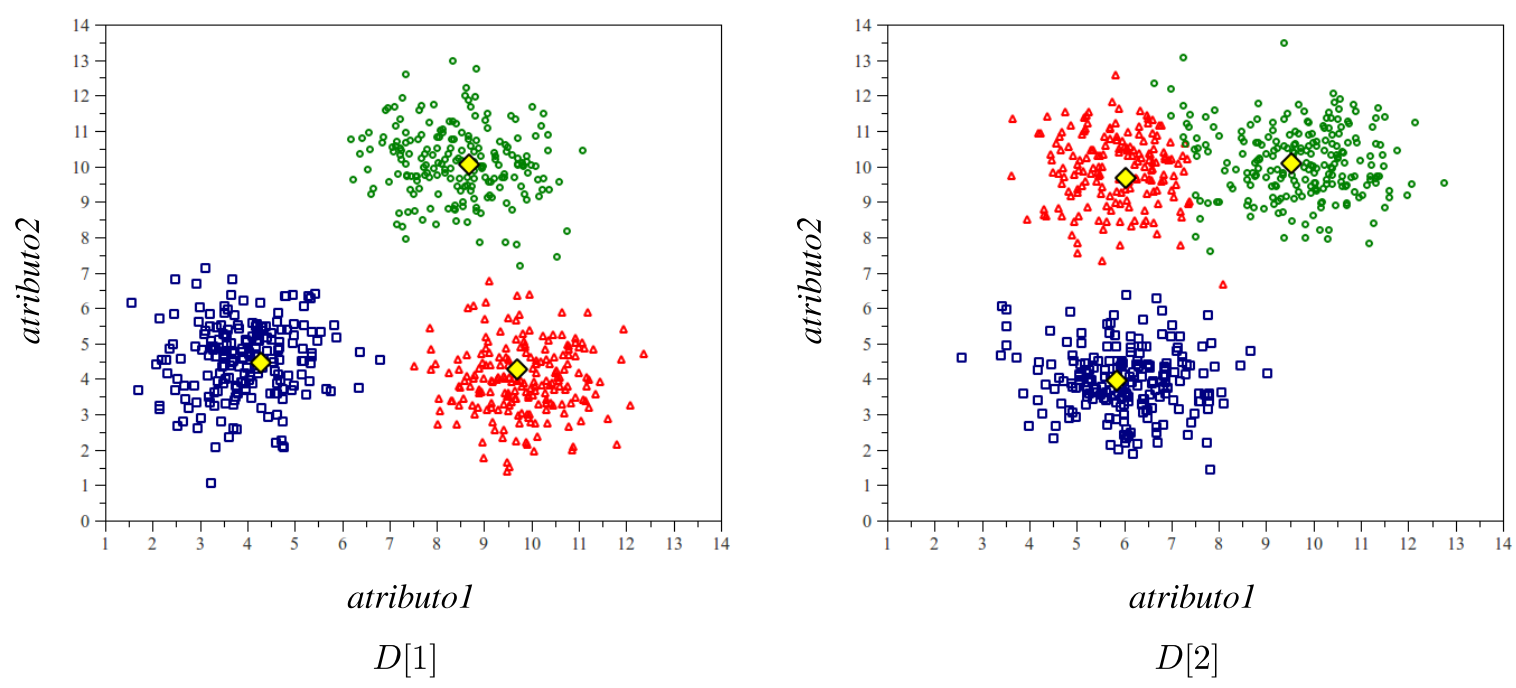

Figura 5.24: Grupos obtidos pelo algoritmo CFC - Conjunto Sin5.
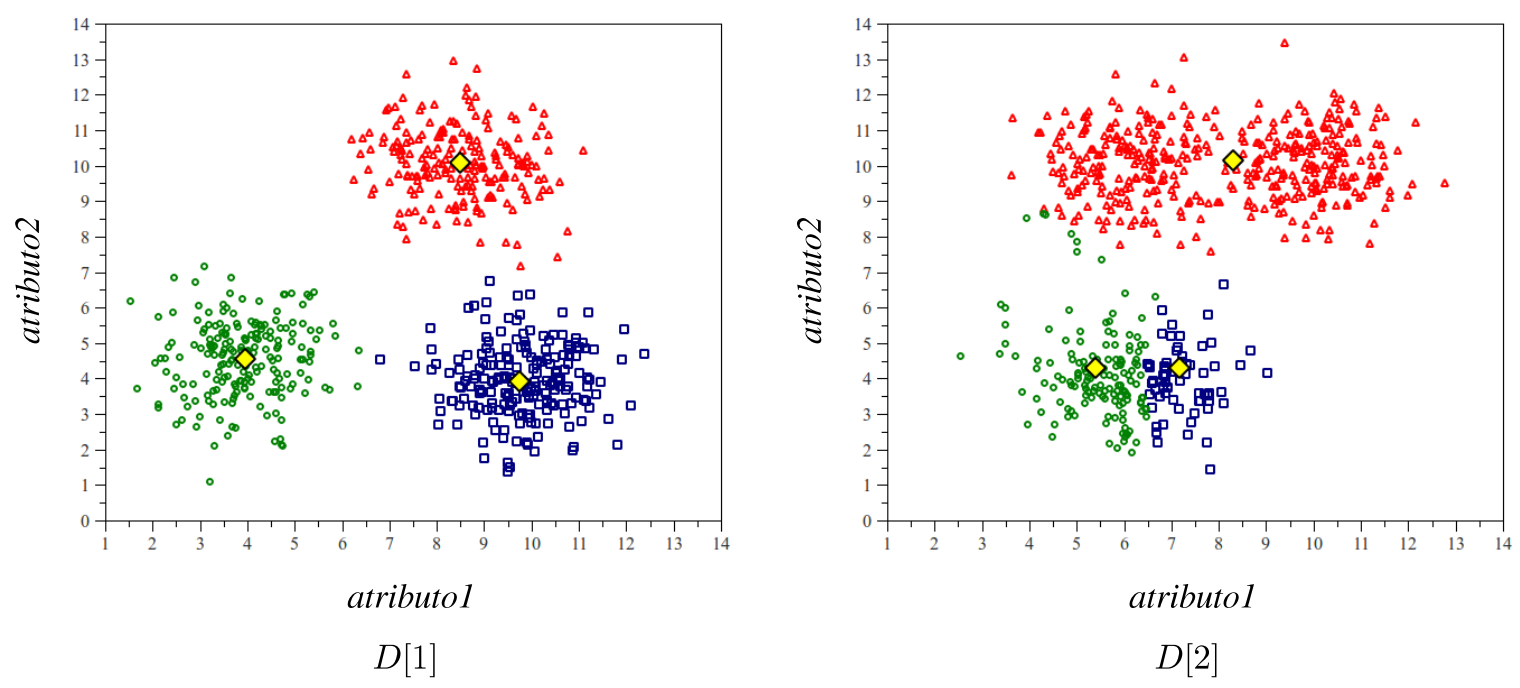

Figura 5.25: Grupos obtidos pelo algoritmo CFC- $\beta_{f}$ - Conjunto Sin5.
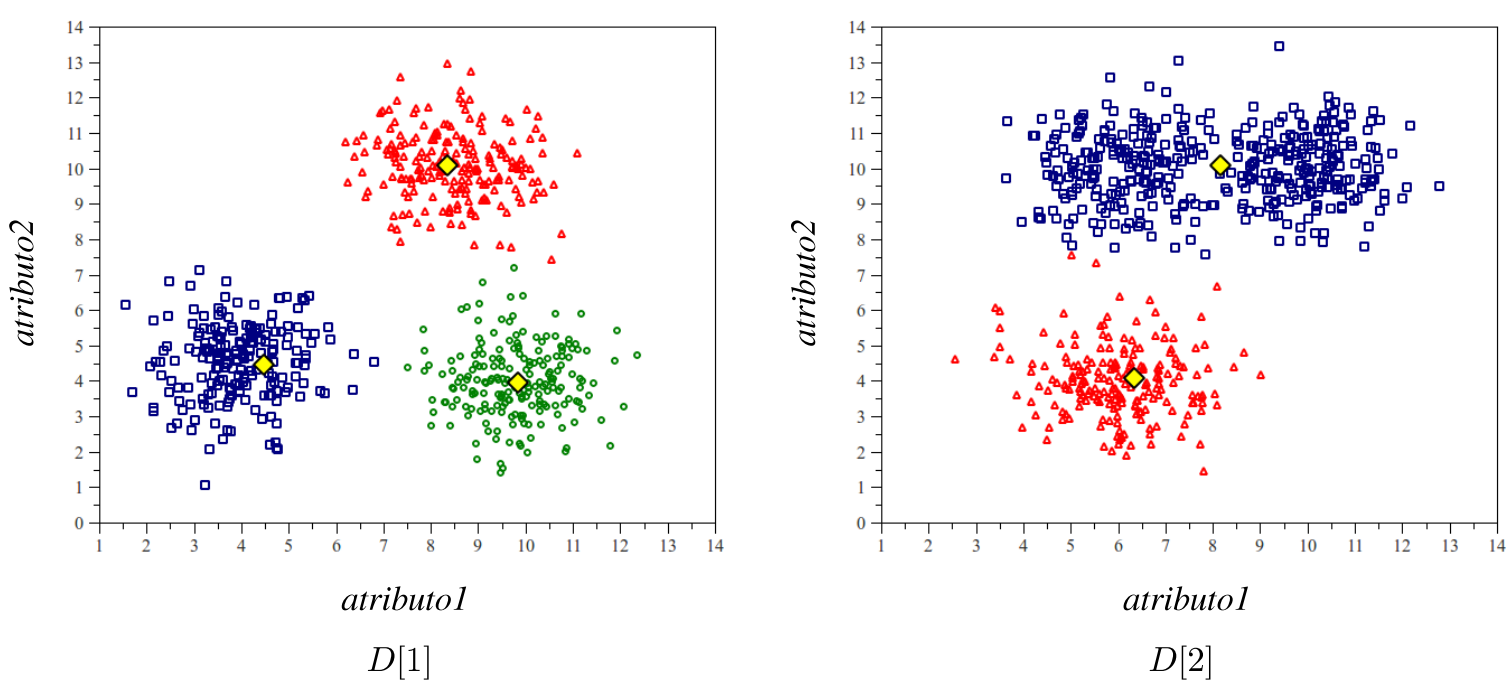

Figura 5.26: Grupos obtidos pelo algoritmo CFCM- $\beta_{f}-c^{*}$ — Conjunto Sin5. 


\subsubsection{Experimentos com Dados Bi-Dimensionais Provenientes de uma Mesma População}

Levando-se em conta a árvore de decisão da Figura 4.3, serão abordados aqui os algoritmos PFCM, PFCM- $c^{*}$ e CFCM- $c^{*}$. Contudo, os resultados alcançados com o algoritmo PFCM (com o número correto de grupos) são naturalmente iguais àqueles obtidos com o algoritmo PFCM- $c^{*}$. Por esta razão, apenas esse último algoritmo (que não requer a informação do número de grupos) foi considerado nessa análise. As Figuras 5.27 e 5.28 ilustram os protótipos obtidos pelo PFCM- $c^{*}$, respectivamente, nos sítios de dados Sin4 e Sin5. Conforme esperado, esses protótipos são precisamente os mesmos que poderiam ser encontrados se os dados estivessem centralizados em um único repositório (ver Figuras 5.20 e 5.23), portanto, representando apropriadamente a suposição de que os dados distribuídos nos diferentes sítios são de uma mesma população. Neste caso, (como esperado) todos os grupos presentes nos dados foram representados em cada um dos sítios. A Figura 5.29, por sua vez, ilustra os grupos encontrados pelo algoritmo $\mathrm{CFCM}-c^{*}$ ao ser aplicado no conjunto Sin4. Os protótipos desses grupos (como esperado) são muito similares àqueles ilustrados na Figura 5.20. Por outro lado, os resultados desse algoritmo para o conjunto Sin 5 merecem explicações adicionais. A Figura 5.30 mostra que quatro grupos (ao invés de três) foram encontrados no sítio $D[1]$ - compare a Figura 5.30 com a Figura 5.23. Dois fatores independentes podem ter contribuído para esse resultado: (i) o algoritmo CFCM- $c^{*}$ assume que o número de grupos pode ser diferente em cada sítio de dados; e (ii) ao sumarizar grupos por meio de seus protótipos (e seus correspondentes pesos) pode haver alguma perda significativa de informação, o que possivelmente pode ocasionar em estimativas não precisas do número de grupos. Note também que, assim como o algoritmo $\mathrm{CFCM}-c^{*}$, o algoritmo PFCM- $c^{*}$ também é baseado na comunicação de protótipos, mas envia os mesmos protótipos para todos os sítios de dados envolvidos no processo de colaboração. Portanto, esse algoritmo mantém implicitamente uma correspondência entre os protótipos de grupos de cada um dos sítios de dados. Essa característica o torna particularmente mais interessante e favorável para o Cenário 2.
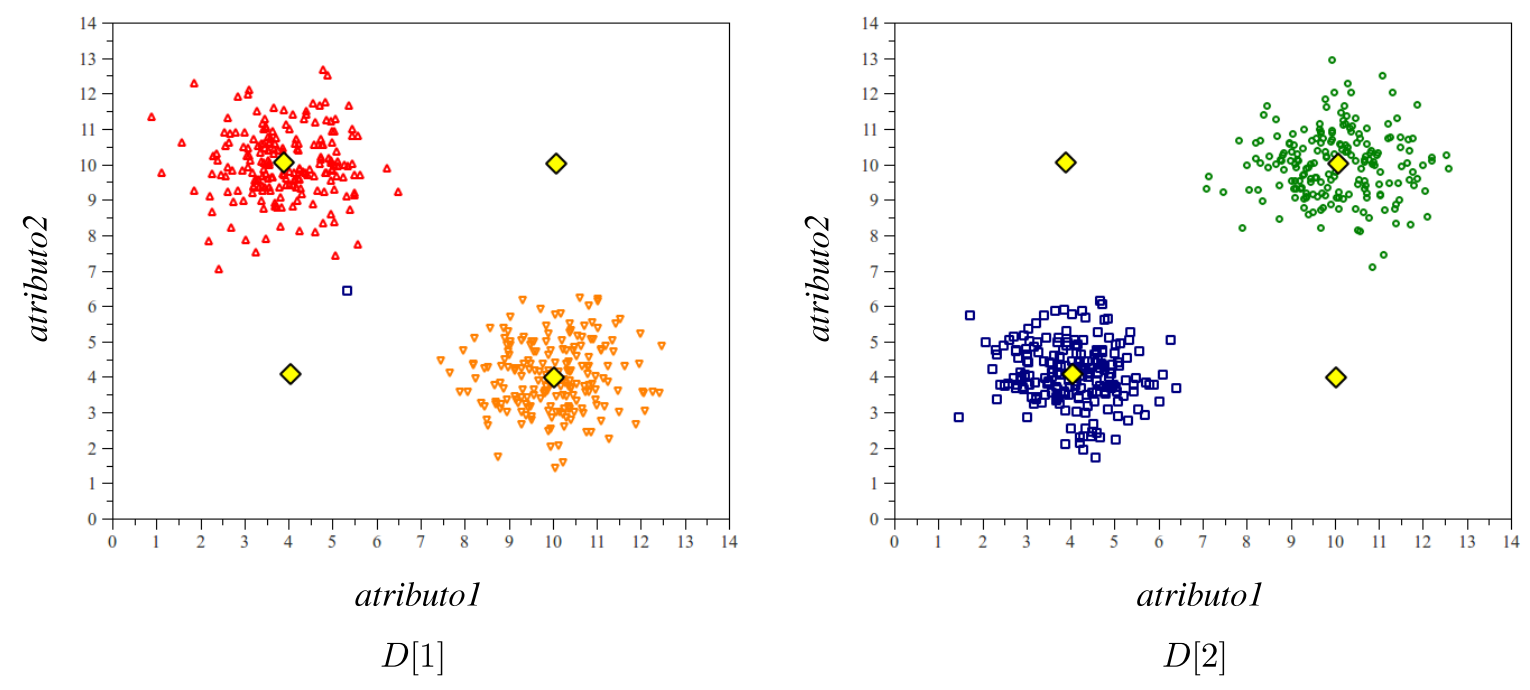

Figura 5.27: Grupos obtidos pelo algoritmo PFCM-c* — Conjunto Sin4. 

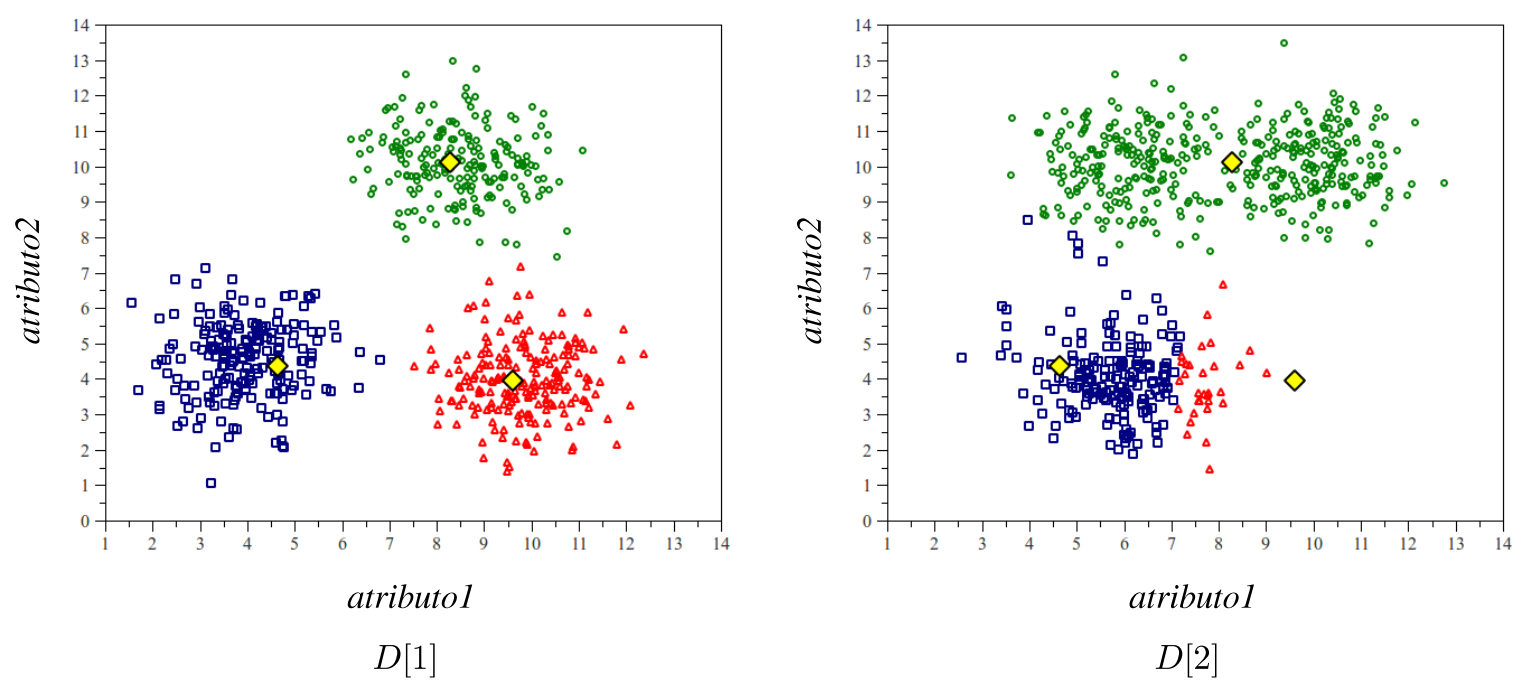

Figura 5.28: Grupos obtidos pelo algoritmo PFCM-c* — Conjunto Sin5.
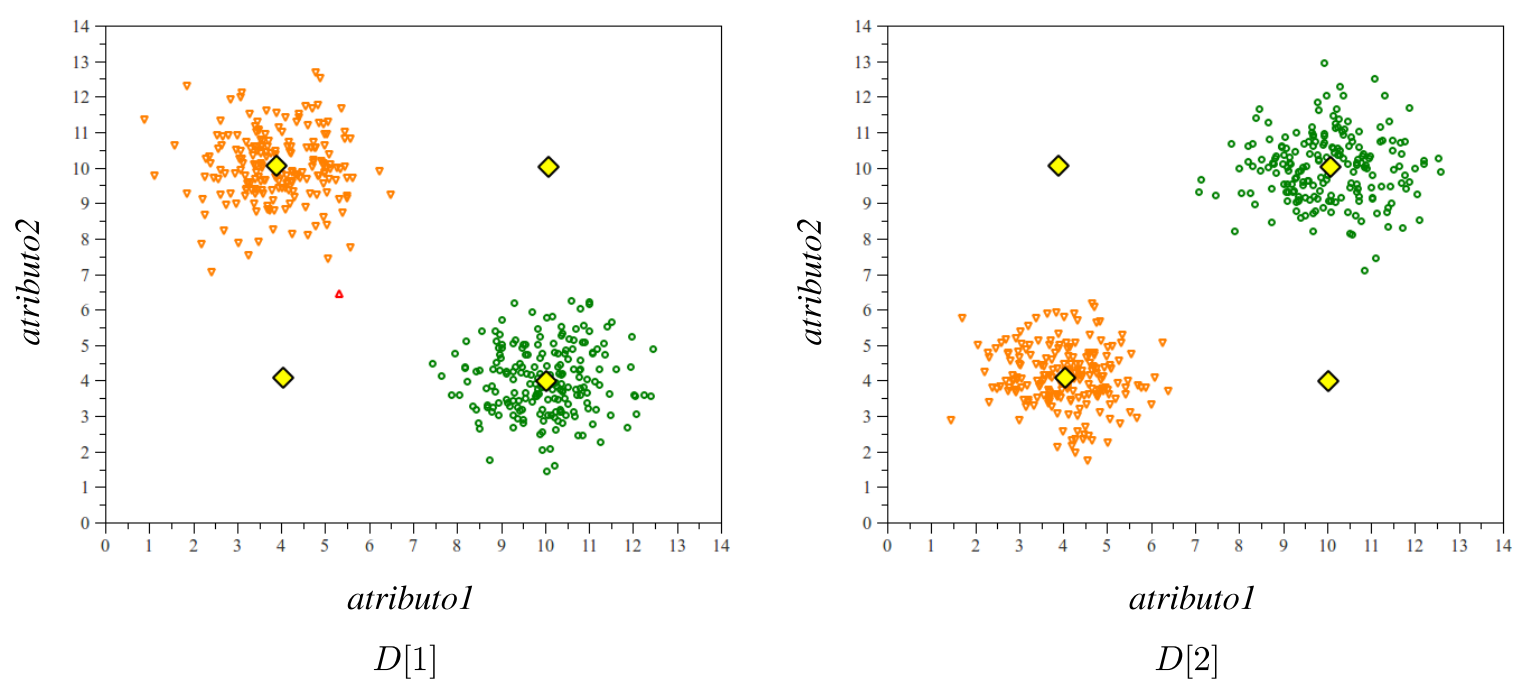

Figura 5.29: Grupos obtidos pelo algoritmo CFCM-c* — Conjunto Sin4.
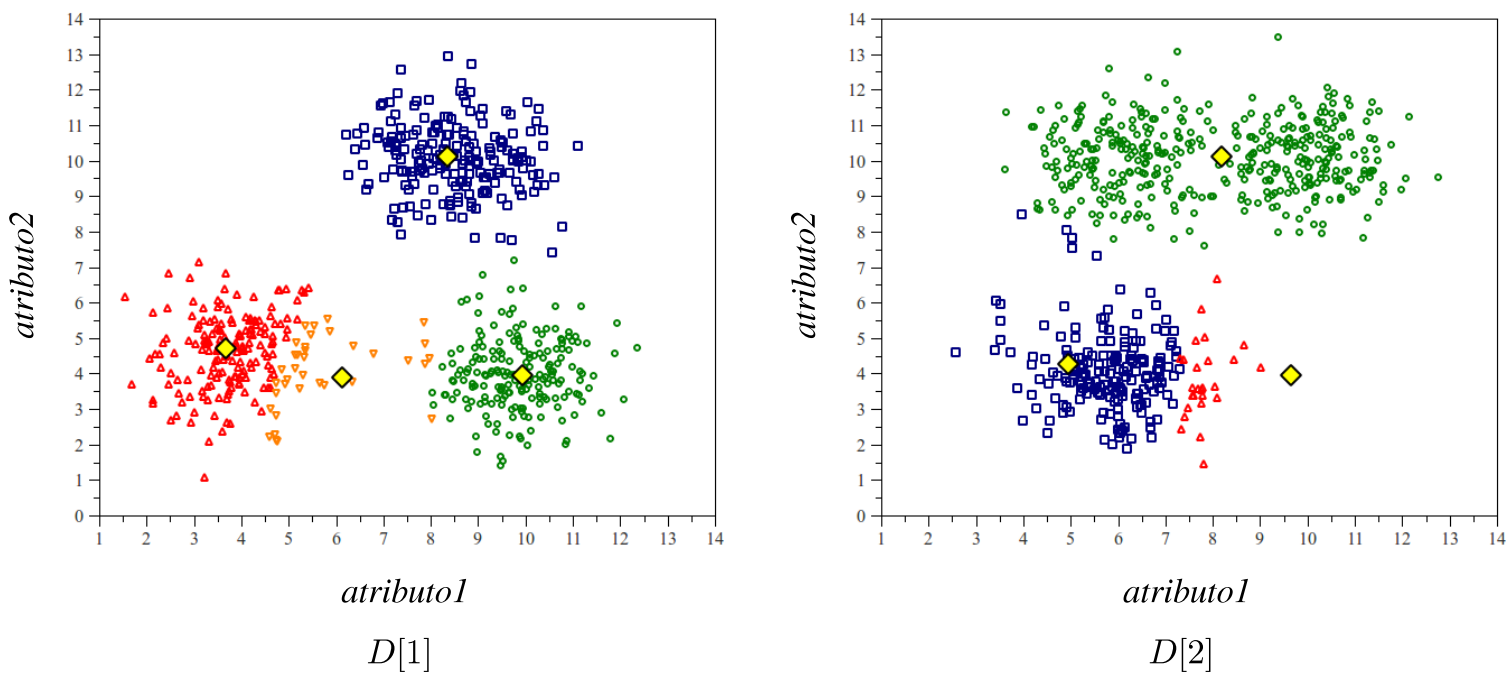

Figura 5.30: Grupos obtidos pelo algoritmo CFCM-c* — Conjunto Sin5. 


\subsubsection{Experimentos Envolvendo Sítios com Dados Completamente Aleatórios}

Dados aleatórios podem afetar negativamente o processo de agrupamento colaborativo, especialmente quando se assume que os dados distribuídos nos diferentes sítios são provenientes de diferentes populações. Nesse cenário, sítios contendo dados aleatórios poderiam prejudicar significativamente os resultados em outros sítios (com estrutura de grupos). Com o objetivo de ilustrar o comportamento dos algoritmos desenvolvidos em tal cenário, o conjunto Sin4 foi modificado. O sítio $D[2]$ desse conjunto foi substituído por um sítio sem estrutura de grupos, composto por 400 objetos gerados aleatoriamente por meio de uma distribuição de probabilidades uniforme dos dados. A Figura 5.31 ilustra os protótipos obtidos pelo algoritmo CFCM-c* $\left(c_{\max }=6\right.$ e $\left.n_{p}=100\right)$ aplicado no conjunto $\operatorname{Sin} 4$ modificado. Esse algoritmo não é dotado de mecanismos apropriados para lidar com dados aleatórios. Dessa forma, nesse exemplo, foram encontrados quatro protótipos no sítio $D[1]$, onde, de fato, deveriam haver apenas dois grupos. Mais precisamente, os protótipos localizados nas coordenadas $(3,02 ; 3,12)$ e $(10,60 ; 10,67)$ em $D[1]$ são apenas um artefato do processo de agrupamento, pois o sítio $D[2]$ não possui estrutura de grupos em seus dados. Neste caso, os dados aleatórios presentes em $D[2]$ prejudicaram o agrupamento em $D[1]$. Este tipo de resultado é justamente o que se espera evitar ao se aplicar algoritmos para agrupamento fuzzy colaborativo em sítios com dados provenientes de diferentes populações (Cenário 1). A Figura 5.32 apresenta os protótipos induzidos pelo algoritmo CFCM- $\beta_{f}-c^{*}\left(c_{\max }=6\right.$ e $\left.n_{p}=100\right)$. Esse algoritmo é menos sensível à dados aleatórios e foi capaz de lidar apropriadamente com esse tipo de problema no conjunto Sin4 modificado. Nesse sentido, como esperado, somente dois grupos foram identificados em $D[1]$.
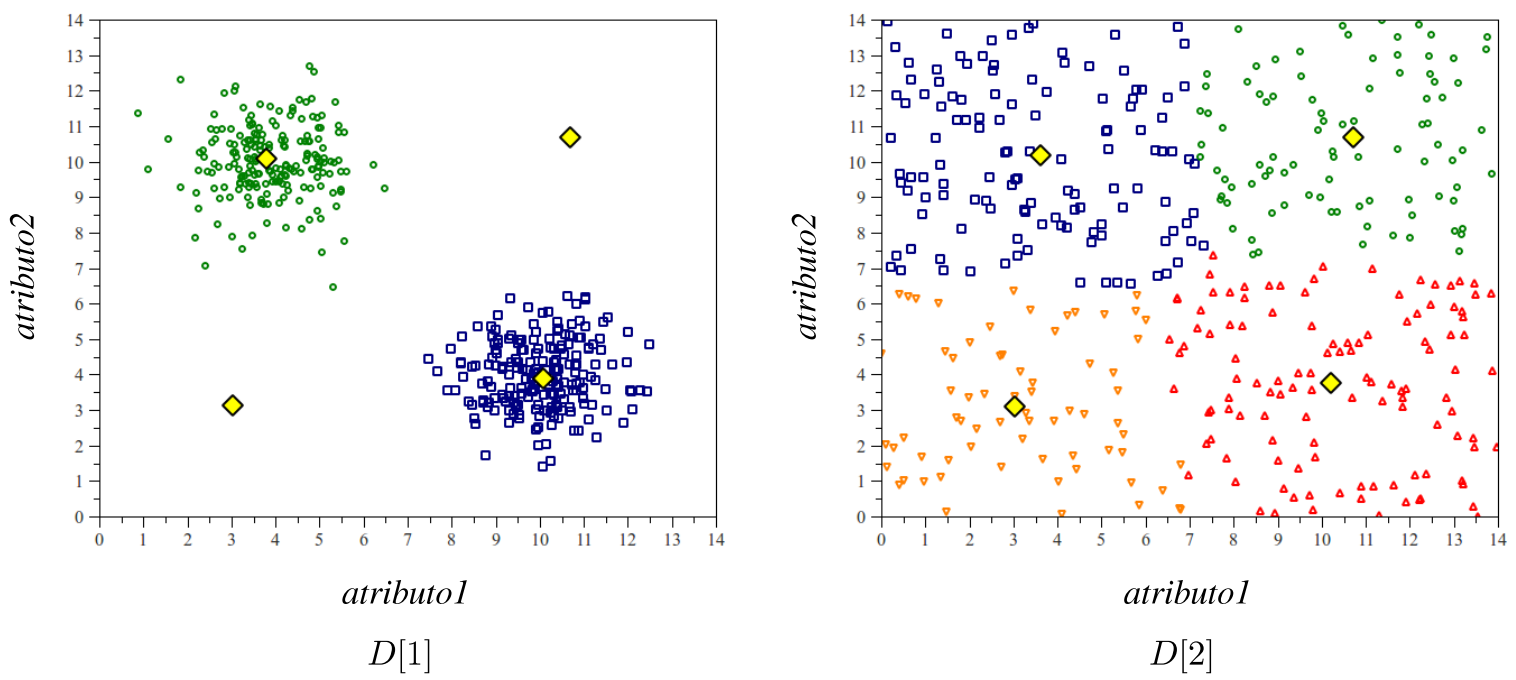

Figura 5.31: Grupos obtidos pelo CFCM-c* — Conjunto Sin4 - D [2] sem estrutura de grupos. 

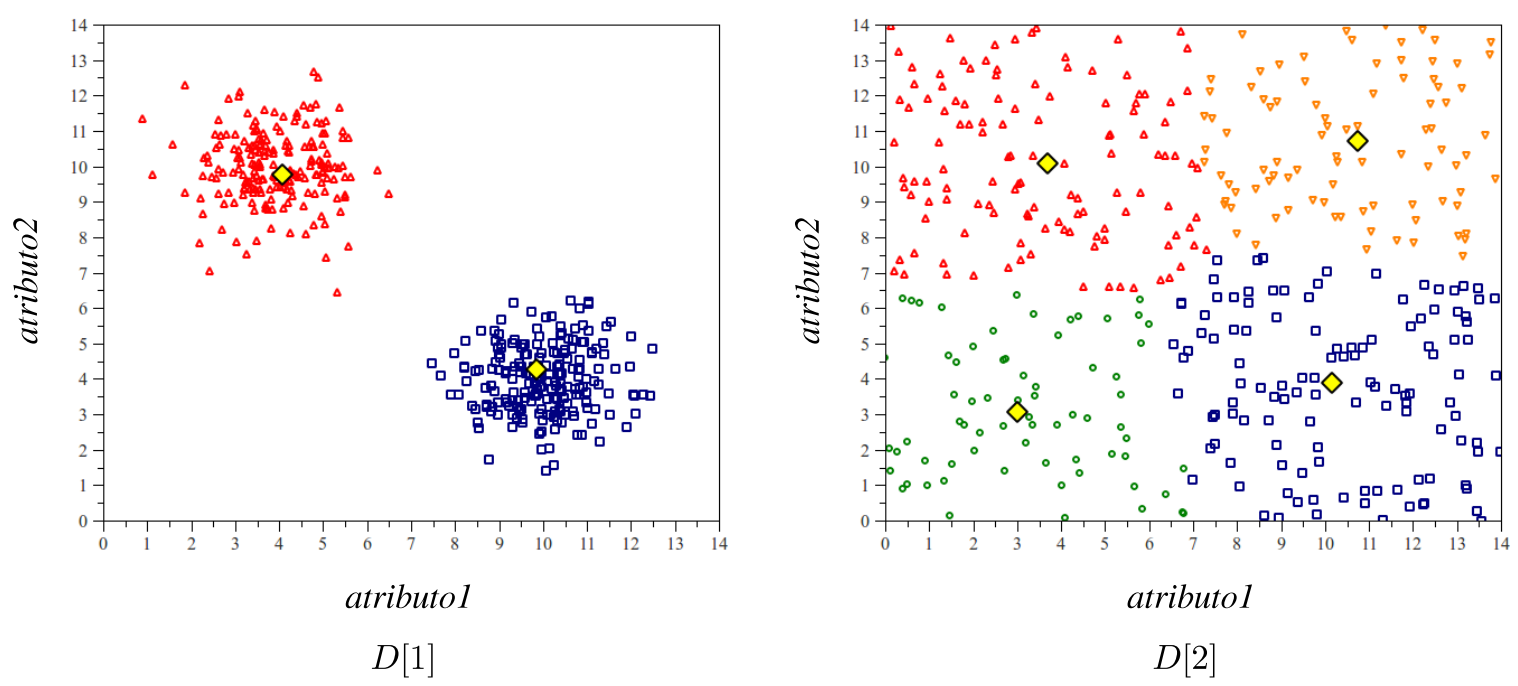

Figura 5.32: Grupos obtidos pelo CFCM- $\beta_{f}-c^{*}-$ Conjunto Sin4 $-D[2]$ sem estrutura de grupos.

\subsubsection{Experimentos Envolvendo Dados Multivariados}

Essa seção envolve uma análise experimental dos algoritmos desenvolvidos em conjuntos de dados multivariados. Nesse sentido, foram usadas aqui modificações dos conjuntos Sin4 e Sin5, inserindo-se novos atributos nesses conjuntos (Seção 5.3.4.1). Além disso, foi usado também o conjunto de dados reais Wisconsin Breast Cancer (Asuncion \& Newman, 2007), o qual é composto por nove atributos (Seção 5.3.4.2).

\subsubsection{Conjunto de Dados Sintéticos}

Nessa seção são reportados os experimentos com os conjuntos de dados Sin 4 e $\operatorname{Sin} 5$ modificados. O conjunto de dados Sin4, em particular, passou a conter oito dimensões com a inserção de seis novos atributos. Em cada um dos sítios de dados ( $D[1]$ e $D[2])$, os 400 objetos foram divididos em dois grupos, sem sobreposição, de acordo com uma mistura de Gaussianas, cujos os vetores médios estão descritos na Tabela 5.5. O algoritmo CFCM- $\beta_{f}-c^{*}$ foi aplicado nesse conjunto (chamado de Sin4-8). A Tabela 5.6 apresenta os protótipos dos dois grupos encontrados por esse algoritmo em cada um dos sítios. Nota-se que esses protótipos são muito similares aos vetores médios descritos na Tabela 5.5, indicando que não houve uma forte colaboração entre os sítios $D[1]$ e $D[2]$, muito provavelmente porque tais sítios são muito diferentes entre si (de fato, o algoritmo obteve $\beta[i i \mid j j]=\beta[j j \mid i i]=0,02$ ). Esse comportamento é esperado para os algoritmos do Cenário 1 (os quais assumem que os dados são de diferentes populações). Nesse sentido, os algoritmos CFC $(\beta=0,10)$, CFC- $\beta_{d}$ e CFC- $\beta_{f}$ (todos com $c=2$ ), obtiveram resultados muito semelhantes aos reportados na Tabela 5.6.

A Tabela 5.7 apresenta os protótipos dos grupos (quatro em cada sítio de dados) encontrados pelo algoritmo CFCM- $c^{*}$. Pode-se notar que os quatro protótipos de cada um dos sítios dessa tabela são muito similares aos quatro vetores médios descritos na Tabela 5.5 (dois do sítio $D[1]$ e outros dois do sítio $D[2]$ ). Esse resultado é esperado para os algoritmos do Cenário 2 (os quais assumem que os dados são de uma mesma população). Nesse sentido, os algoritmos 
Tabela 5.5: Vetores médios representantes dos grupos em $D[1]$ e $D[2]$ — Conjunto Sin4-8.

\begin{tabular}{|c|c|c|c|c|c|c|c|c|}
\hline \multicolumn{7}{|c|}{$D[1]$} \\
\hline$c 1$ & 10,00 & 4,00 & 7,00 & 5,00 & 6,00 & 4,00 & 5,00 & 5,00 \\
\hline$c 2$ & 4,00 & 10,00 & 4,00 & 2,00 & 2,00 & 1,00 & 5,00 & 5,00 \\
\hline \multicolumn{8}{|c|}{$D[2]$} \\
\hline$c 1$ & 10,00 & 10,00 & 10,00 & 8,00 & 11,00 & 9,00 & 5,00 & 5,00 \\
\hline$c 2$ & 4,00 & 4,00 & 14,00 & 12,00 & 14,00 & 13,00 & 5,00 & 5,00 \\
\hline
\end{tabular}

Tabela 5.6: Protótipos de grupos obtidos pelo algoritmo CFCM- $\beta_{f}-c^{*}-$ Conjunto Sin4-8.

\begin{tabular}{|c|c|c|c|c|c|c|c|c|}
\hline \multicolumn{7}{|c|}{$D[1]$} \\
\hline$c 1$ & 9,93 & 4,09 & 7,18 & 5,11 & 6,16 & 4,19 & 4,99 & 5,00 \\
\hline$c 2$ & 3,92 & 9,97 & 3,97 & 2,04 & 2,00 & 1,02 & 5,01 & 5,01 \\
\hline \multicolumn{8}{|c|}{$D[2]$} \\
\hline$c 1$ & 9,95 & 9,88 & 9,84 & 7,87 & 10,83 & 8,79 & 4,97 & 4,94 \\
\hline$c 2$ & 4,06 & 4,09 & 14,01 & 12,00 & 13,96 & 12,94 & 4,98 & 4,99 \\
\hline
\end{tabular}

PFCM (com $c=4)$ e PFCM- $c^{*}$ obtiveram também resultados muito similares aos reportados na Tabela 5.7. Esses experimentos mostram que, tanto para os algoritmos do Cenário 1, quanto para os algoritmos do Cenário 2, os resultados foram semelhantes aos obtidos ao se usar o conjunto Sin4 (original, com apenas dois atributos). Levando-se em conta os dois primeiros atributos (segunda e terceira coluna dessas tabelas), ao se comparar os protótipos da Tabela 5.6 com os da Figura 5.21 e os protótipos da Tabela 5.7 com os das Figuras 5.27 e 5.29, observa-se que os algoritmos apresentaram comportamentos semelhantes àqueles previamente observados para dados bi-dimensionais, conforme esperado.

Tabela 5.7: Protótipos de grupos obtidos pelo algoritmo CFCM- $c^{*}$ - Conjunto Sin4-8.

\begin{tabular}{|c|c|c|c|c|c|c|c|c|}
\hline \multicolumn{10}{|c|}{$D[1]$} \\
\hline$c 1$ & 10,01 & 3,99 & 7,05 & 4,97 & 5,98 & 3,99 & 4,99 & 5,00 \\
\hline$c 2$ & 3,91 & 9,98 & 3,96 & 2,03 & 1,98 & 1,00 & 5,01 & 5,01 \\
\hline$c 3$ & 10,04 & 9,98 & 9,98 & 8,00 & 11,04 & 8,98 & 4,97 & 4,94 \\
\hline$c 4$ & 4,06 & 4,08 & 14,02 & 12,01 & 13,97 & 12,95 & 4,98 & 4,99 \\
\hline \multicolumn{8}{|c|}{$D[2]$} \\
\hline$c 1$ & 10,01 & 3,99 & 7,05 & 4,97 & 5,98 & 3,99 & 4,99 & 5,00 \\
\hline$c 2$ & 3,91 & 9,98 & 3,96 & 2,03 & 1,98 & 1,00 & 5,01 & 5,01 \\
\hline$c 3$ & 10,04 & 9,98 & 9,98 & 8,00 & 11,04 & 8,98 & 4,97 & 4,94 \\
\hline$c 4$ & 4,06 & 4,08 & 14,02 & 12,01 & 13,97 & 12,95 & 4,98 & 4,99 \\
\hline
\end{tabular}

A Tabela 5.8 descreve os vetores médios (representantes dos grupos) do conjunto de dados Sin5 (modificado), o qual, após a inserção de dois novos atributos, passou a conter quatro dimensões. Para cada sítio de dados $(D[1]$ e $D[2])$, foi mantido o particionamento dos 600 objetos em três grupos, conservando-se as sobreposições existentes do conjunto original. A Tabela 5.9 apresenta os protótipos dos dois grupos encontrados ao aplicar o algoritmo FCM- $c^{*}\left(c_{\max }=6 \mathrm{e}\right.$ $\left.n_{p}=100\right)$ nos dados centralizados $(D[1] \cup D[2])$. Uma explicação plausível para os resultados reportados nessa tabela parte da constatação que, em quatro dimensões, tanto a complexidade do problema quanto o nível de sobreposição entre os grupos aumentam.

Ao aplicar o algoritmo CFCM- $\beta_{f}-c^{*}$ no conjunto Sin5-4 foram identificados em $D[1]$ e em $D[2]$, respectivamente, três e dois grupos (ver Tabela 5.10). O sítio $D[1]$, em particular, apresentou protótipos muito semelhantes aos seus vetores médios, descritos na Tabela 5.8. Por outro 
Tabela 5.8: Vetores médios representantes dos grupos em $D[1]$ e $D[2]$ - Conjunto Sin5-4.

\begin{tabular}{|c|c|c|c|c|}
\hline \multicolumn{5}{|c|}{$D[1]$} \\
\hline$c 1$ & 10,00 & 4,00 & 5,00 & 6,00 \\
\hline$c 2$ & 4,00 & 4,50 & 3,00 & 2,00 \\
\hline$c 3$ & 8,50 & 10,00 & 10,00 & 11,00 \\
\hline \multicolumn{5}{|c|}{$D[2]$} \\
\hline$c 1$ & 10,00 & 10,00 & 8,00 & 10,00 \\
\hline$c 2$ & 6,00 & 4,00 & 3,00 & 4,00 \\
\hline$c 3$ & 6,00 & 10,00 & 12,00 & 12,00 \\
\hline
\end{tabular}

Tabela 5.9: Protótipos de grupos obtidos pelo algoritmo FCM- $c^{*}-\operatorname{Sin} 5-4(D[1] \cup D[2])$.

\begin{tabular}{|c|c|c|c|c|}
\hline \multicolumn{5}{|c|}{$D[1] \cup D[2]$} \\
\hline$c 1$ & 8,16 & 10,04 & 9,99 & 11,03 \\
\hline$c 2$ & 6,49 & 4,16 & 3,63 & 3,97 \\
\hline
\end{tabular}

lado, o sítio $D[2]$ passou a conter dois grupos, os quais são representados por protótipos muito similares aos descritos na Tabela 5.9. Nota-se que os protótipos da Tabela 5.10 (considerandose apenas os dois primeiros atributos) são muito similares aos protótipos encontrados na Figura 5.26. Da mesma forma e nas mesmas condições, outros algoritmos do Cenário 1, como o CFC e o CFC- $\beta_{f}$, obtiveram resultados semelhantes aos ilustrados, respectivamente, nas Figuras 5.24 e 5.25. Essa constatação reforça a suposição de que esses algoritmos mantiveram o mesmo desempenho na presença de dados multivariados.

Finalmente, ao se aplicar o algoritmo PFCM- $c^{*}$ no conjunto Sin5-4 foram encontrados dois grupos em cada um dos sítios (ver Tabela 5.11). Nessa tabela, os protótipos, em cada um dos sítios, são exatamente iguais aos protótipos apresentados na Tabela 5.9. Esse comportamento é esperado para os algoritmos do Cenário 2, sendo desejável que esses algoritmos apresentem resultados tão próximos quanto possível dos resultados obtidos se os dados estivessem centralizados em um mesmo repositório. Os algoritmos PFCM (com $c=2$ ) e CFCM- $c^{*}$, também mais apropriados para o Cenário 2, apresentaram resultados muito similares aos reportados na Tabela 5.11.

Tabela 5.10: Protótipos de grupos obtidos pelo algoritmo CFCM- $\beta_{f}-c^{*}-$ Conjunto Sin5-4.

\begin{tabular}{|c|c|c|c|c|}
\hline \multicolumn{5}{|c|}{$D[1]$} \\
\hline$c 1$ & 9,81 & 3,97 & 4,95 & 5,99 \\
\hline$c 2$ & 4,28 & 4,48 & 3,07 & 2,33 \\
\hline$c 3$ & 8,34 & 10,06 & 10,02 & 11,04 \\
\hline \multicolumn{5}{|c|}{$D[2]$} \\
\hline$c 1$ & 8,02 & 10,03 & 9,98 & 11,04 \\
\hline$c 2$ & 6,29 & 4,04 & 3,30 & 4,06 \\
\hline
\end{tabular}

Tabela 5.11: Protótipos de grupos obtidos pelo algoritmo PFCM-c* — Conjunto Sin5-4.

\begin{tabular}{|c|c|c|c|c|}
\hline \multicolumn{5}{|c|}{$D[1]$} \\
\hline$c 1$ & 8,16 & 10,04 & 9,99 & 11,03 \\
\hline$c 2$ & 6,49 & 4,16 & 3,63 & 3,97 \\
\hline \multicolumn{5}{|c|}{$D[2]$} \\
\hline$c 1$ & 8,16 & 10,04 & 9,99 & 11,03 \\
\hline$c 2$ & 6,49 & 4,16 & 3,63 & 3,97 \\
\hline
\end{tabular}




\subsubsection{Conjunto de Dados Reais — Wisconsin Breast Cancer}

O conjunto de dados Wisconsin Breast Cancer (Asuncion \& Newman, 2007) consiste de 683 objetos descritos por nove atributos. Cada um desses objetos pode assumir uma das duas classes possíveis (benigno ou maligno), havendo 444 objetos da classe benigno e 239 da classe maligno. Ao aplicar o algoritmo FCM- $c^{*}\left(c_{\max }=6\right.$ e $\left.n_{p}=100\right)$ nesse conjunto (sem os rótulos de classe) obteve-se como resultado protótipos muito semelhantes aos centróides (vetores médios) de cada uma das classes. Portanto, essas classes podem ser adequadamente modeladas por meio de dois grupos, o que motivou o uso desse conjunto de dados no presente problema de agrupamento. Nesse sentido, os objetos foram, então, distribuídos em dois sítios $(D[1]$ e $D[2])$ por meio de uma amostragem aleatória e estratificada dos dados. Ao usar os algoritmos do Cenário 2 (dados provenientes de uma mesma população) foram encontrados dois grupos em cada um dos sítios de dados. As distâncias Euclidianas entre os protótipos obtidos pelos algoritmos CFCM- $c^{*}$ e PFCM- $c^{*}$ em relação aos correspondentes protótipos encontrados pelo algoritmo FCM- $c^{*}$ (aplicado nos dados centralizados $-D[1] \cup D[2]$ ) foram 0,2 e 0 , respectivamente. Essas pequenas diferenças entre os protótipos são, de fato, esperadas. Ou seja, os protótipos obtidos por esses algoritmos são essencialmente os mesmos que teriam sido obtidos se os dados estivessem centralizados em um único repositório.

Quando assume-se que os dados são provenientes de diferentes populações (Cenário 1), as análises quantitativas são mais difíceis de serem realizadas. Em resumo, todos os algoritmos adequados para esse cenário também encontraram dois grupos em cada um dos sítios de dados. As distâncias Euclidianas entre os protótipos obtidos pelos algoritmos CFC, CFC- $\beta_{d}$, e CFC- $\beta_{f}$ $(c=2)$ em relação aos respectivos protótipos encontrados pelo algoritmo FCM- $c^{*}$ (aplicado em $D[1] \cup D[2]$ ) foram todas iguais a 2,1. Analogamente, o respectivo valor para o algoritmo CFCM- $\beta_{f}-c^{*}$ foi 2,2 . Ao comparar esses resultados com os obtidos pelos algoritmos do $\mathrm{Ce}$ nário 2, tais valores são (de certa forma) também esperados. Os algoritmos do Cenário 1, em particular, buscam ajustar os protótipos levando-se em conta que os dados são provenientes de diferentes populações. Nesse sentido, o grau dos ajustes tende a ser menor do que aqueles presenciados no Cenário 2, o que pode explicar as maiores distâncias observadas em relação aos protótipos encontrados pelo algoritmo FCM- $c^{*}$ nos dados centralizados. Dessa forma, a comparação relativa entre os algoritmos do Cenário 1 é difícil de ser realizada, pois não se é capaz de saber (a priori) quais seriam os resultados esperados após o processo de colaboração realizado por esses algoritmos (e.g., não se sabe quais seriam os protótipos finais após o processo de colaboração).

\subsection{Considerações Finais}

As análises experimentais realizadas, bem como os exemplos ilustrativos, permitiram deduzir uma série de conclusões a respeito das características particulares dos algoritmos desenvolvidos e estudados nesse trabalho. Em resumo, as análises da Seção 5.2 mostraram que os algoritmos CFC, CFC- $\beta_{d}$ e CFC- $\beta_{f}$ realizam apenas pequenos ajustes nos protótipos de grupos durante o processo de colaboração. A partir desses experimentos também se notou a dificul- 
dade em se definir iterativamente o valor ótimo de $\beta$ no algoritmo CFC. Levando isso em conta, os algoritmos CFC- $\beta_{d}$ e CFC- $\beta_{f}$ são mais recomendados, pois estimam os níveis de interação automaticamente a partir dos dados. O algoritmo $\mathrm{CFC}-\beta_{f}$, em particular, computa uma única vez os níveis de interação entre os sítios de dados, sendo, portanto, mais indicado do que o CFC- $\beta_{d}$ (que faz isso a cada estágio de colaboração). Contudo, esses algoritmos necessitam da informação do número de grupos $(c)$, o qual é fixo para todos os sítios de dados. Por outro lado, os algoritmos CFCM- $c^{*}$ e CFCM- $\beta_{f}-c^{*}$ são capazes de estimar automaticamente o valor de $c$ para cada um dos sítios de dados (conforme ilustram os experimentos da Seção 5.3). Tal característica é mais interessante para o Cenário 1, no qual assume-se que os dados distribuídos são de diferentes populações. Nesse sentido, para esse cenário, o algoritmo CFCM- $\beta_{f}-c^{*}$ é o mais indicado, sobretudo por ser mais robusto à dados aleatórios (Seção 5.3.3). Ao assumir que os dados são de uma mesma população (Cenário 2) e o número de grupos seja desconhecido, o algoritmo PFCM- $c^{*}$ é o mais indicado, pois apresentou maior conformidade com as suposições sobre os dados, além de possuir uma complexidade de tempo assintótica menor do que a do algoritmo CFCM-c*. Contudo, caso o número de grupos seja conhecido, pode-se utilizar o algoritmo PFCM, que é computacionalmente mais eficiente que os algoritmos PFCM-c* e CFCM- $c^{*}$. Ao lidar com dados multivariados (Seção 5.3.4), os algoritmos apresentaram desempenho similar aos observados em experimentos com conjuntos de dados bi-dimensionais. No entanto, enquanto que para os algoritmos do Cenário 2 o resultado final pode ser conhecido (resultados dos algoritmos devem ser tão próximos quanto possíveis do resultado obtido caso os dados estivessem centralizados), para os algoritmos do Cenário 1 essa informação não é conhecida. Em resumo, a comparação relativa entre os algoritmos do Cenário 1 são difíceis de serem realizadas, pois não se sabe ( a priori) quais seriam os resultados esperados após o processo de colaboração dos algoritmos.

Algumas outras importantes considerações levam em conta o nível de interação $\beta[i i \mid j j]$ (Seção 4.3.1) e o índice de validade SFS (Seção 2.4.4). Mais especificamente, o nível de interação $\beta[i i \mid j j]$, pela própria definição da Eq. (4.10), pode não ser adequado quando os sítios envolvidos na colaboração possuem diferentes números de grupos. As funções objetivo em (2.3) e (4.9) expressam o erro quadrático e não são apropriadas para a comparação relativa de resultados de agrupamento com diferentes números de grupos. Claramente essas funções tendem a produzir valores mais baixos (para um mesmo conjunto de dados) com o aumento do número de grupos. Contudo, não foram observados efeitos negativos desta limitação nos experimentos realizados com o algoritmo CFCM- $\beta_{f}-c^{*}$, que é o único algoritmo sujeito a este tipo de problema. Além disso, essa limitação pode produzir níveis de interação mais baixos, o que é correto ao se considerar que, de fato, há agrupamentos bem diferentes (com diferentes números de grupos). Com relação ao índice de validade SFS, em algumas situações, os protótipos comunicados por outros sítios (na forma de objetos virtuais no sítio local) podem se constituir de grupos singletons. Em outras palavras, um objeto virtual (protótipo comunicado por outro sítio) pode se tornar um grupo de um único objeto. Nesse caso, o índice SFS atribui (como penalização) valor de silhueta igual a 0 para esse objeto. No entanto, na verdade, esse objeto (virtual) representa um grupo 
de um outro sítio (que pode não ser singleton). Esse fato pode fazer com que a SFS subestime o número de grupos, mas em nenhum dos experimentos realizados esse tipo de problema ocorreu. 


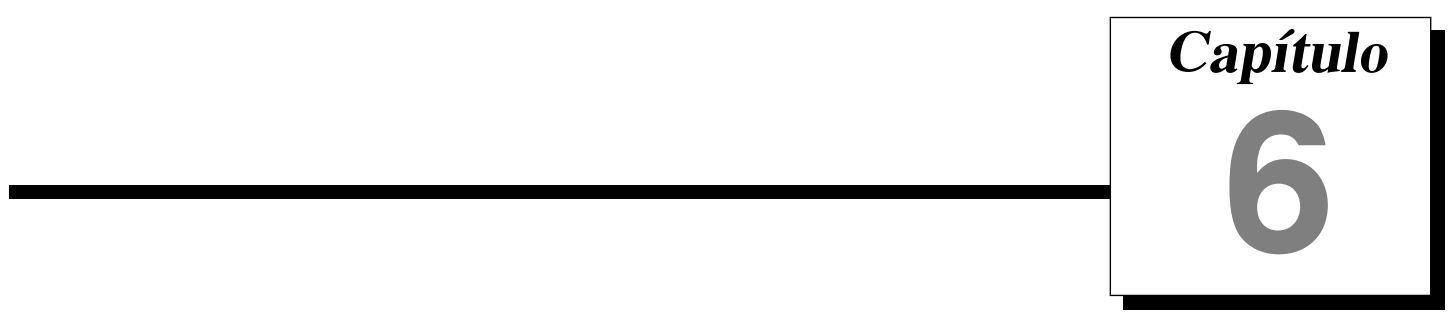

\section{Conclusão e Trabalhos Futuros}

As técnicas de mineração de dados, bem como o processo de KDD, têm desempenhado um importante papel em diversas áreas do conhecimento humano (Han \& Kamber, 2006; Tan et al., 2006; Chen et al., 1996; Fayyad et al., 1996c). Mais recentemente, essas ferramentas têm encontrado espaço em um novo e complexo domínio, no qual os dados a serem minerados estão fisicamente distribuídos. Esse novo domínio ocasionou o surgimento de um novo campo de estudo denominado de descoberta de conhecimento distribuído, ou Distributed Knowledge Discovery (DKD) (Zhang et al., 2004; Johnson \& Kargupta, 2000). Em DKD, alguns algoritmos específicos para agrupamento de dados podem ser utilizados (Silva \& Klusch, 2006; Merugu \& Ghosh, 2005; Strehl \& Ghosh, 2002) - em particular algumas variantes do conhecido algoritmo Fuzzy C-Means (FCM) (Bezdek, 1981; Dunn, 1973), as quais têm sido investigadas sob o nome de agrupamento colaborativo (Pedrycz \& Rai, 2008; Pedrycz, 2007, 2002). Esse trabalho teve como objetivo estudar e superar algumas das limitações encontradas em dois desses algoritmos. Nesse sentido, as principais contribuições desse trabalho estão descritas na Seção 6.1. A Seção 6.2, por sua vez, aborda as conclusões do trabalho e, finalmente, na Seção 6.3 são apresentadas as sugestões de trabalhos futuros.

\subsection{Principais Contribuições}

Com o objetivo de superar algumas das limitações encontradas nos algoritmos CFC (Pedrycz \& Rai, 2008) e PFCM (Rahimi et al., 2004), cinco novos algoritmos para agrupamento fuzzy colaborativo foram desenvolvidos nesse trabalho. Mais precisamente, foram estudadas e propostas algumas melhorias para o algoritmo $\mathrm{CFC}$, o qual depende de dois parâmetros que devem ser informados pelo usuário (o nível de interação $\beta$ e o número de grupos $c$ ). Com o objetivo de suprimir a necessidade do primeiro parâmetro $(\beta)$, foram desenvolvidos dois novos 
algoritmos, chamados de CFC- $\beta_{d}$ e CFC- $\beta_{f}$. Esses algoritmos estimam automaticamente os níveis de interação entre os sítios de dados. No que concerne ao último parâmetro $(c)$, foram desenvolvidos dois novos algoritmos, o CFCM- $c^{*}$ e o CFCM- $\beta_{f}-c^{*}$, capazes de estimar o número de grupos automaticamente para cada um dos sítios de dados. O algoritmo PFCM, por sua vez, tem se mostrado potencialmente útil para agrupamento colaborativo, especialmente em cenários nos quais se assume que os dados distribuídos são de uma mesma população. Contudo, esse algoritmo também necessita da informação do número de grupos $(c)$. Para superar essa limitação, foi desenvolvida uma extensão, chamada de PFCM- $c^{*}$, capaz de estimar automaticamente o valor de $c$. Por fim, foi realizado um estudo desses algoritmos em cenários específicos de aplicação. Na prática, dependendo das suposições sobre os dados (i.e., dados que são de uma mesma ou de diferentes populações), bem como da dificuldade do usuário em definir parâmetros, alguns algoritmos podem ser mais vantajosos do que outros. A partir disso, foi sugerida uma árvore de decisão para o usuário interessado em escolher dentre os algoritmos disponíveis o mais adequado para o problema que se tem em mãos. As complexidades de tempo, espaço e comunicação dos algoritmos também foram investigadas e podem auxiliar nessa tarefa de escolha dos algoritmos.

Durante o trabalho de Mestrado dois artigos foram publicados (Coletta et al., 2010; Vendramin et al., 2011). O primeiro artigo (Coletta et al., 2010) foi iniciado durante o trabalho de iniciação científica, em tema relacionado ao abordado na dissertação, e concluído durante o Mestrado. O segundo artigo (Vendramin et al., 2011), por sua vez, apresenta parte das idéias sobre agrupamento colaborativo desenvolvidas nessa pesquisa (em co-autoria com o grupo de pesquisa envolvido nesse trabalho). Finalmente, os principais resultados obtidos no presente trabalho foram sumarizados em um artigo que foi submetido para avaliação em um periódico internacional indexado. Esse artigo, no qual o aluno é o primeiro autor, foi desenvolvido em parceria com o aluno Lucas Vendramin e com os Professores Eduardo R. Hruschka e Ricardo J. G. B. Campello, ambos do ICMC/USP, bem como com o Prof. Witold Pedrycz, da University of Alberta, Canada.

\subsection{Conclusões}

As análises experimentais e os exemplos ilustrativos produzidos permitiram deduzir uma série de conclusões a respeito das características particulares dos algoritmos desenvolvidos e estudados nesse trabalho. Em geral, o estudo realizado pode ser útil para o usuário interessado em aplicar algoritmos para agrupamento fuzzy colaborativo na prática. Em particular, quando se assume que os dados são de diferentes populações (Cenário 1 ) e o número de grupos é $a$ priori conhecido (e o mesmo para todos os sítios), o algoritmo CFC- $\beta_{f}$ é o mais indicado, pois estima (apenas uma única vez) de maneira automática os níveis de interação entre os sítios. Por outro lado, caso o número de grupos não seja conhecido, o algoritmo CFCM- $\beta_{f}-c^{*}$ é o indicado e o único disponível para essa situação no Cenário 1. Esse algoritmo é, naturalmente, o mais interessante para esse cenário, pois além de estimar o número de grupos para cada um 
dos sítios de dados, é mais robusto à dados aleatórios. Ao assumir que os dados são de uma mesma população (Cenário 2) e o número de grupos não é conhecido, o algoritmo PFCM-c*é o preferido por estar em maior conformidade com as suposições sobre os dados e possuir uma complexidade de tempo assintótica menor que a do algoritmo $\mathrm{CFCM}-c^{*}$. Contudo, caso o número de grupos seja conhecido pode-se utilizar o algoritmo PFCM que é computacionalmente mais eficiente que os algoritmos PFCM- $c^{*}$ e CFCM- $c^{*}$. Embora existam várias medidas para se avaliar resultados de agrupamento (incluindo diversos critérios de validade), não existem ainda metodologias consolidadas para se avaliar algoritmos para agrupamento fuzzy colaborativo. De maneira mais específica, não há ainda um consenso fundamentado sobre quais deveriam ser os resultados esperados da aplicação de um algoritmo desse tipo em diversos sítios de dados (em especial, quando os dados são provenientes de diferentes populações - Cenário 1). Dessa forma, enquanto que para os algoritmos do Cenário 2 os resultados finais podem ser conhecidos (resultados dos algoritmos deveriam ser tão próximos quanto possível do resultado obtido caso os dados estivessem centralizados), para os algoritmos do Cenário 1 não se tem essa informação. Nesse sentido, a comparação relativa entre os algoritmos do Cenário 1 são difíceis de serem realizadas por não se saber qual deveria ser o resultado final após o processo de colaboração.

\subsection{Trabalhos Futuros}

O estudo de medidas quantitativas para a comparação relativa dos algoritmos para agrupamento fuzzy colaborativo complementaria o trabalho realizado e é promissor - particularmente para os algoritmos do Cenário 1. Outro trabalho futuro interessante envolve considerar mais informações no processo de agrupamento colaborativo, como o volume, a densidade e a orientação dos grupos. Tais informações poderiam ser comunicadas entre os sítios de dados, compartilhando-se, assim, um maior conhecimento sobre as estruturas presentes nos mesmos. Outras dinâmicas de comunicação (ou topologias de comunicação) também poderiam ser estudadas. Por exemplo, durante o processo de colaboração, ao invés de todos os sítios se comunicarem com todos os outros, poderia haver uma comunicação unidirecional (com um sítio se comunicando com outro, mas este outro não se comunicando com o primeiro). Seguindo essa idéia, poderia se estudar uma topologia de comunicação na forma de anel, na qual cada sítio se comunica com o seu subsequente até que o último sítio se comunica novamente com o primeiro. Outros cenários poderiam ser investigados também, como, por exemplo, a colaboração entre partições de dados induzidas em diferentes instantes de tempo e a colaboração entre partições de dados com diferentes graus de especificidade (quanto maior o número de grupos maior o grau de especificidade). Por fim, poderiam ser conduzidos estudos mais aprofundados sobre a robustez dos algoritmos na presença de dados aleatórios, bem como na presença de ruídos. 


\section{Referências Bibliográficas}

Anderberg, M. (1973). Cluster analysis for applications. Academic Press.

Asuncion, A.; Newman, D. (2007). UCI machine learning repository. http://www. ics . uci.edu/ mlearn/MLRepository.html.

Bezdek, J. C. (1981). Pattern Recognition with Fuzzy Objective Function Algorithms. Kluwer Academic Publishers.

Bigus, J. P. (1996). Data mining with neural networks: solving business problems from application development to decision support. McGraw-Hill, Inc.

Bishop, C. M. (2007). Pattern Recognition and Machine Learning - Information Science and Statistics. N. 1. Springer.

Boutsinas, B.; Gnardellis, T. (2002). On distributing the clustering process. Pattern Recognition Letters, v.23, n.8, p.999-1008.

Brachman, R. J.; Anand, T. (1996). The process of knowledge discovery in databases. Advances in knowledge discovery and data mining, p. 37-57. American Association for Artificial Intelligence.

Campello, R.; Hruschka, E. (2006). A fuzzy extension of the silhouette width criterion for cluster analysis. Fuzzy Sets and Systems, v.157, n.21, p.2858-2875.

Campello, R.; Hruschka, E.; Alves, V. (2009). On the efficiency of evolutionary fuzzy clustering. Journal of Heuristics, v.15, p.43-75.

Chen, M.-S.; Han, J.; Yu, P. S. (1996). Data mining: An overview from a database perspective. IEEE Trans. on Knowl. and Data Eng., v.8, n.6, p.866-883.

Coletta, L.; Hruschka, E.; Covoes, T.; Campello, R. (2010). Fuzzy clustering-based filter. Information Processing and Management of Uncertainty in Knowledge-Based Systems. Theory and Methods, v. 80 de Communications in Computer and Information Science, p. 406-415. Springer Berlin/Heidelberg. 
Da Silva, J.; Giannella, C.; Bhargava, R.; Kargupta, H.; Klusch, M. (2005). Distributed data mining and agents. Engineering Applications of Artificial Intelligence, v.18, p.791-807.

Da Silva, J. C.; Klusch, M. (2007). Privacy Preserving Pattern Discovery in Distributed Time Series. p. 207-214. IEEE International Conference on Data Engineering Workshop.

Datta, S.; Bhaduri, K.; Giannella, C.; Wolff, R.; Kargupta, H. (2006). Distributed data mining in peer-to-peer networks. IEEE Internet Computing, v.10, p.18-26.

Datta, S.; Giannella, C.; Kargupta, H. (2009). Approximate distributed k-means clustering over a peer-to-peer network. IEEE Trans. on Knowl. and Data Eng., v.21, p.1372-1388.

Dempster, A. P.; Laird, N. M.; Rubin, D. B. (1977). Maximum likelihood from incomplete data via the em algorithm. Journal of the Royal Statistical Society, Series B, v.39, n.1, p.1-38.

Dhillon, I.; Modha, D. (2000). A data-clustering algorithm on distributed memory multiprocessors. Large-Scale Parallel Data Mining, v. 1759 de Lecture Notes in Computer Science, p. 802-802. Springer Berlin / Heidelberg.

Dimitriadou, E.; Weingessel, A.; Hornik, K. (2001). Voting-merging: An ensemble method for clustering. Artificial Neural Networks, ICANN 2001, v. 2130 de Lecture Notes in Computer Science, p. 217-224. Springer Berlin / Heidelberg.

Dumitrescu, D.; Lazzerini, B.; Jain, L. C. (2000). Fuzzy sets and their application to clustering and training. CRC Press, Inc.

Dunn, J. (1973). A fuzzy relative of the isodata process and its use in detecting compact wellseparated clusters. Journal of Cybernetics, v.3, p.32-57.

Everitt, B. S.; Landau, S.; Leese, M. (2001). Cluster Analysis. N. 4. Wiley Publishing.

Falcón, R.; Depaire, B.; Vanhoof, K.; Abraham, A. (2008). Towards a suitable reconciliation of the findings in collaborative fuzzy clustering. Proceedings of the 2008 Eighth International Conference on Intelligent Systems Design and Applications, p. 652-657. IEEE Computer Society.

Falcon, R.; Jeon, G.; Bello, R.; Jeong, J. (2007). Learning collaboration links in a collaborative fuzzy clustering environment. MICAI 2007: Advances in Artificial Intelligence, v. 4827 de Lecture Notes in Computer Science, p. 483-495. Springer Berlin / Heidelberg.

Fayyad, U. M.; Piatetsky-Shapiro, G.; Smyth, P.; Uthurusamy, R. (1996a). American Association for Artificial Intelligence.

Fayyad, U. M.; Piatetsky-Shapiro, G.; Smyth, P.; Uthurusamy, R. (1996b). From data mining to knowledge discovery: an overview. Advances in knowledge discovery and data mining, $\mathrm{p}$. 1-37. American Association for Artificial Intelligence. 
Fayyad, U. M.; Piatetsky-Shapiro, G.; Smyth, P.; Uthurusamy, R. (1996c). Preface. Advances in knowledge discovery and data mining, p. xiii-xiv. American Association for Artificial Intelligence.

Forestier, G.; Gançarski, P.; Wemmert, C. (2010). Collaborative clustering with background knowledge. Data \& Knowledge Engineering, v.69, n.2, p.211-228.

Forestier, G.; Wemmert, C.; Gançarski, P. (2008). Semi-supervised collaborative clustering with partial background knowledge. 2008 IEEE International Conference on Data Mining Workshops, p. 211-217.

Fred, A. L. N.; Jain, A. K. (2005). Combining multiple clusterings using evidence accumulation. IEEE transactions on pattern analysis and machine intelligence, v.27, n.6, p.835-50.

Frossyniotis, D. S.; Pateritsas, C.; Stafylopatis, A. (2005). A multi-clustering fusion scheme for data partitioning. International Journal of Neural Systems, v.15, p.391-401.

Ghaemi, R.; Sulaiman, N.; Ibrahim, H.; Mustapha, N. (2009). A Survey: Clustering Ensembles Techniques. Proceedings of World Academy of Science, Engineering and Technology, v.38.

Halkidi, M.; Batistakis, Y.; Vazirgiannis, M. (2001). On clustering validation techniques. Journal of Intelligent Information Systems, v.17, p.107-145.

Han, J.; Kamber, M. (2006). Data Mining. Concepts and Techniques. N. 2. Morgan Kaufmann.

Hansen, P.; Jaumard, B. (1997). Cluster analysis and mathematical programming. Mathematical Programming, p. 79: 191-215.

Hartigan, J. (1975). Clustering algorithms. John Wiley and Sons.

Hore, P.; Hall, L.; Goldgof, D. (2009). A scalable framework for cluster ensembles. Pattern Recognition, v.42, n.5, p.676-688.

Horta, D.; Naldi, M.; Campello, R.; Hruschka, E.; de Carvalho, A. (2009). Evolutionary fuzzy clustering: An overview and efficiency issues. Foundations of Computational Intelligence, v. 204 de Studies in Computational Intelligence, p. 167-195. Springer Berlin / Heidelberg.

Hruschka, E. R.; Campello, R. J.; de Castro, L. N. (2006). Evolving clusters in gene-expression data. Information Sciences, v.176, n.13, p.1898-1927.

Höppner, F.; Klawonn, F.; Kruse, R.; Rurkler, T. (1999). Fuzzy Cluster Analysis: Methods for Classification, Data Analysis and Image Recognition. Wiley.

Jain, A.; Murty, M.; Flynn, P. (1999). Data clustering: A review. ACM Computing Surveys, v.31, p.264-323.

Jain, A. K. (2010). Data clustering: 50 years beyond k-means. Pattern Recognition Letters, v.31, n.8, p.651 - 666 . 
Jain, A. K.; Dubes, R. C. (1988a). Algorithms for clustering data. Prentice-Hall, Inc.

Jain, A. K.; Dubes, R. C. (1988b). Algorithms for clustering data, chapter Cluster validity, p. 142-222. Prentice-Hall, Inc.

Johnson, E.; Kargupta, H. (2000). Collective, hierarchical clustering from distributed, heterogeneous data. Lecture Notes in Computer Science, v.1759, p.221-244.

Kargupta, H.; Chan, P. (2000). Advances in Distributed and Parallel Knowledge Discovery. MIT Press.

Kargupta, H.; Huang, W.; Sivakumar, K.; Johnson, E. (2001). Distributed clustering using collective principal component analysis. Knowledge and Information Systems, v.3, p.422448.

Kaufman, L.; Rousseeuw, P. (1990). Finding Groups in Data: An Introduction to Cluster Analysis. Wiley Interscience.

Kaufmann, L.; Rousseeuw, P. J. (1987). Clustering by means of medoids. Statistical Data Analysis based on the L1 Norm, p. 405-416.

Klösgen, W.; Zytkow, J. M. (1996). Knowledge discovery in database terminology. Advances in Knowledge Discovery and Data Mining, p. 573-592.

Klusch, M.; Lodi, S.; Moro, G. (2003). Distributed clustering based on sampling local density estimates. International joint conference on artificial intelligence, v.18, p.485-490.

Kwok, T.; Smith, K.; Lozano, S.; Taniar, D. (2002). Parallel fuzzy c- means clustering for large data sets. Euro-Par 2002 Parallel Processing, v. 2400 de Lecture Notes in Computer Science, p. 27-58. Springer Berlin / Heidelberg.

Liu, C. L. (1968). Introduction to Combinatorial Mathematics (Computer Science Series). Mcgraw-Hill College.

MacQueen, J. B. (1967). Some methods for classification and analysis of multivariate observations. Proc. of the fifth Berkeley Symposium on Mathematical Statistics and Probability, v. 1, p. 281-297. University of California Press.

Merugu, S.; Ghosh, J. (2005). A privacy-sensitive approach to distributed clustering. Pattern Recognition Letters, v.26, n.4, p.399-410.

Milligan, G. W. (1996). Clustering validation: Results and implications for applied analysis, chapter Clustering and Classification, p. 341-375. Word Scientific.

Mitchell, T. M. (1997). Machine Learning. McGraw-Hill.

Mitra, S.; Banka, H.; Pedrycz, W. (2006). Rough-fuzzy collaborative clustering. IEEE Transactions on Systems Man and Cybernetics-Part B-Cybernetics, v.36, p.795-805. 
Modenesi, M.; Costa, M.; Evsukoff, A.; Ebecken, N. (2007). Parallel fuzzy c-means cluster analysis. High Performance Computing for Computational Science - VECPAR 2006, v. 4395 de Lecture Notes in Computer Science, p. 52-65. Springer Berlin / Heidelberg.

Nguyen, N.; Caruana, R. (2007). Consensus clusterings. Proceedings of the 2007 Seventh IEEE International Conference on Data Mining, p. 607-612. IEEE Computer Society.

Park, B.; Kargupta, H.; Johnson, E.; Sanseverino, E.; Hershberger, D.; Silvestre, L. (2002). Distributed, collaborative data analysis from heterogeneous sites using a scalable evolutionary technique. Applied Intelligence, v.16, n.1, p.19-42.

Park, B. H.; Kargupta, H. (2002). Distributed Data Mining: Algorithms, Systems, and Applications. Data Mining Handbook, p. 341-358. Lawrence Earlbaum Associates.

Pedrycz, W. (2002). Collaborative fuzzy clustering. Pattern Recogn. Lett., v.23, n.14, p.16751686.

Pedrycz, W. (2007). Collaborative and knowledge-based fuzzy clustering. International Journal of Innovative Computing, Information and Control, v.3, n.1, p.1-12.

Pedrycz, W.; Gomide, F. (2007). Fuzzy Systems Engineering: Toward Human-Centric Computing. Wiley-IEEE Press.

Pedrycz, W.; Rai, P. (2008). Collaborative clustering with the use of fuzzy c-means and its quantification. Fuzzy Sets and Systems, v.159, n.18, p.2399-2427.

Piatetsky-Shapiro, G.; Frawley, W. J. (1991). Knowledge Discovery in Databases. AAAI/MIT Press.

Rahimi, S.; Zargham, M.; Thakre, A.; Chhillar, D. (2004). A parallel fuzzy c-mean algorithm for image segmentation. IEEE Annual Meeting of the Fuzzy Information, 2004. Processing NAFIPS '04., p. 234-237.

Sharan, R.; Shamir, R. (2000). Center click: A clustering algorithm with applications to gene expression analysis. Proceedings of the Eighth International Conference on Intelligent Systems for Molecular Biology, p. 307-316. AAAI Press.

Silva, J. D.; Klusch, M. (2006). Inference in distributed data clustering. Engineering Applications of Artificial Intelligence, v.19, n.4, p.363 - 369.

Steinbach, M.; Karypis, G.; Kumar, V. (2000). A comparison of document clustering techniques. KDD workshop on text mining, v.400, p.1-2.

Strehl, A.; Ghosh, J. (2002). Cluster Ensembles - A Knowledge Reuse Framework for Combining Multiple Partitions. Journal of Machine Learning Research, v.3, p.583-617. 
Symon, M. J. (1977). Clustering criterion and multi-variate normal mixture. Biometrics, v.77, p.35-43.

Tan, P. N.; Steinbach, M.; Kumar, V. (2006). Introduction to Data Mining. Capítulo 8: Cluster Analysis: Basic Concepts and Algorithms. Addison-Wesley.

Tian, J.; Zhu, L.; Zhang, S.; Liu, L. (2005). Improvement and parallelism of k-means clustering algorithm. Tsinghua Science and Technology, v.10, n.3, p.277 - 281.

Topchy, A.; Jain, A.; Punch, W. (2004). A mixture model for clustering ensembles. Proc. SIAM Data Mining, p. 379-390. Citeseer.

Topchy, A.; Jain, A.; Punch, W. (2005). Clustering ensembles: models of consensus and weak partitions. IEEE Transactions on Pattern Analysis and Machine Intelligence, v.27, n.12, p. $1866-1881$.

Tsoumakas, G. (2004). Clustering classifiers for knowledge discovery from physically distributed databases. Data \& Knowledge Engineering, v.49, p.223-242.

Tumer, K.; Agogino, A. K. (2008). Ensemble clustering with voting active clusters. Pattern Recognition Letters, v.29, n.14, p.1947 - 1953.

Vendramin, L.; Campello, R.; Coletta, L.; Hruschka, E. (2011). Distributed fuzzy clustering with automatic detection of the number of clusters. International Symposium on Distributed Computing and Artificial Intelligence, v. 91 de Advances in Intelligent and Soft Computing, p. 133-140. Springer Berlin / Heidelberg.

Vendramin, L.; Campello, R. J. G. B.; Hruschka, E. R. (2009). On the comparison of relative clustering validity criteria. Proceedings of the SIAM International Conference on Data Mining, p. 733-744.

Vendramin, L.; Campello, R. J. G. B.; Hruschka, E. R. (2010). Relative clustering validity criteria: A comparative overview. Stat. Anal. Data Min., v.3, n.4, p.209-235.

Witten, I. H.; Frank, E. (2005). Data mining: practical machine learning tools and techniques. N. 2 in Morgan Kaufmann Series in Data Management Systems. Morgan Kaufmann.

Xie, X.; Beni, G. (1991). A validity measure for fuzzy clustering. Pattern Analysis and Machine Intelligence, IEEE Transactions on, v.13, n.8, p.841 -847.

Xu, R.; Wunsch, D. (2009). Clustering. Wiley-IEEE Press.

Yu, F.; Tang, J.; Cai, R. (2007). Partially Horizontal Collaborative Fuzzy C-Means. International Journal of Fuzzy Systems, v.9, p.198-204.

Yu, F.; Yu, J.; Tang, J. (2009). The model of generalized partially horizontal collaborative fuzzy c-means. Control and Decision Conference, 2009. CCDC '09. Chinese, p. 6095-6099. 
Zeng, Y.; Tang, J.; Garcia-Frias, J.; Gao, G. R. (2002). An adaptive meta-clustering approach: Combining the information from different clustering results. Proceedings of the IEEE Computer Society Conference on Bioinformatics, CSB '02, p. 276-. IEEE Computer Society.

Zhang, S.; Zhang, C.; Wu, X. (2004). Knowledge Discovery in Multiple Databases. SpringerVerlag. 